\title{
Review on Triggered Liposomal Drug Delivery with a Focus on Ultrasound
}

\author{
Hesham G. Moussa $^{\mathrm{a}}$, Ana M. Martins ${ }^{\mathrm{b}}$ and Ghaleb A. Husseini ${ }^{\mathrm{b},}$ \\ ${ }^{a}$ Department of Electrical Engineering, American University of Sharjah, PO Box 26666; Sharjah, United \\ Arab Emirates; ${ }^{b}$ Department of Chemical Engineering, American University of Sharjah, PO Box 26666, \\ Sharjah, United Arab Emirates
}

\begin{abstract}
Chemotherapy is widely used for cancer treatment; however, it causes unwanted side effects in patients. To avoid these adverse effects, nanocarriers have been developed, which can be loaded with the chemotherapeutic agents, directed to the cancer site and, once there, are exposed to stimuli that will trigger the drug release.
\end{abstract}

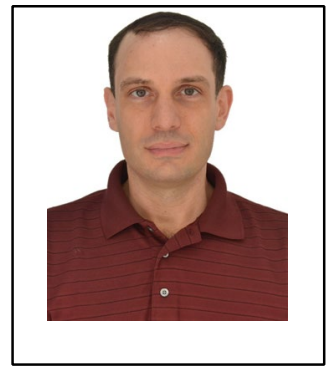

G.A. Husseini

Liposomes can be chemically modified to increase their circulation time, their stability, and their sensitivity to specific stimulus. Additionally, ligands can be conjugated to their surface, allowing for their specific binding to receptors overexpressed on the surface of cancer cells and the subsequent internalization via endocytosis. Using a triggering mechanism, including temperature, ultrasound, enzymes or a change in $\mathrm{pH}$, the release of the drug is controlled and induced inside the cells, hence avoiding drug release in systemic circulation, which in turn reduces the undesired side effects of conventional chemotherapy. Ultrasound has been widely studied as a drug release trigger from liposomes, due to its wellknown physics and previous uses in medicine.

This review focuses on liposome-based drug delivery systems, using different trigger mechanisms, with a focus on ultrasound. The physical mechanisms of ultrasound release are also investigated and the results of in vitro and in vivo studies are summarized.

Keywords: Drug delivery systems, liposomes, nanocarriers, triggered delivery, ultrasound.

\section{INTRODUCTION}

The past decade witnessed an increased interest in the area of drug delivery especially for the treatment of cancer, an often terminal disease. In 2012, cancer was reported as the leading cause of death in Europe and North America. It was also regarded as the second leading cause of death in the less developed countries, especially in Africa. Statistics of estimated cancer incidence, mortality and prevalence worldwide are well documented by GLOBOCAN, a project of the International Agency for Research on Cancer, World Health Organization (WHO) [1, 2]. Unfortunately, this disease can be very difficult to treat, and sometimes even impossible. Tumors can strike in two forms, either malignant or benign. In both forms, tumors result from an abnormal growth of cells. In the case of benign tumors, this abnormal growth usually forms a stable, non-moving mass that can be removed from the body through surgery. On the contrary, in the case of malignant tumors, i.e., cancer, the growing cells can spread in the body, causing metastasis, increasing the number of affected organs and interfering with vital body functions.

When a cancerous tumor is detected, it is possible to remove the whole tumor if it is still contained within a specific area. However, in the case of metastasis, it may not be feasible to do so [3]. In some cases, no surgery is possible, especially when the tumor is located in inaccessible or vital areas (e.g. brain tumors). These facts led medical doctors and scientists to try to develop assistive treatments that can help in stopping cancer from spreading inside the body. The German chemist Paul Ehrlich was the first to use the term chemotherapy in the 1900s [4]. He was also the first to use animal models for testing newly developed chemical medicines. These pioneering discoveries lead to the true revolution of chemotherapeutic agents used for cancer treatment as we know it today [4]. However, it was not until the 1960s that chemical medicines were considered effective against cancer. Prior to that, surgery and radiotherapy were dominant in the treatment of cancer. In 1960, scientists discovered that the use of chemotherapy, in addition to surgery, increased the possibility for a successful recovery of cancer patients [4]. However, chemotherapy, although a great discovery, can also cause several health problems to those undergoing treatment. This is due to the fact that the chemicals used are not selective, i.e., they affect not only cancerous cells, but also healthy cells in the body. Hence, patients treated with anti-neoplastic chemical agents suffer from several serious side effects due to the death of vital healthy cells, such as hair loss, fever, fatigue, weight loss and lowered immunity $[1,3]$.

\footnotetext{
*Address correspondence to this author at the Department of Chemical Engineering, American University of Sharjah, PO Box 26666, Sharjah, UAE; Tel: +971-6-515 2970; Fax: +971-6-5152979;

E-mail: ghusseini@aus.edu
}

Since scientists believe in the essentiality of using chemotherapy for cancer treatment, researchers directed considerable attention to reducing its side effects. One 
approach consisted of carefully checking the vitals of the patient and testing for allergic reactions, before he/she was declared eligible to undergo chemotherapeutic treatment [3]. However, the greatest advances in this field were, possibly, due to the collective work of medical scientists and engineers who used their technological and scientific knowledge to design efficient Drug Delivery Systems (DDS).

Nanoparticles are an essential part of a DDS. They have a durable chemical composition that is compatible with the bodily fluids, and can be designed to circulate in the body for extended periods, reaching up to several days [5]. The role of these nanoparticles is to encapsulate the chemotherapeutic agent in their core, so that it does not interact with healthy cells in the body. Nanoparticles are designed based on two targeting methods, passive and active, both of which make them capable of accumulating in cancerous tumors preferentially. This, in turn, reduces the dosage that would be needed to treat the tumors if the free drug was to be used. Once the nanoparticles reach the diseased site, external or internal means of triggering are used to release their content, by disrupting their membrane, which causes the drug to leak out. Hence, the release trigger is the complementary part of many DDS. Examples of nanoparticles are liposomes, micelles, dendrimers, archaeosomes, solid lipid nanoparticles and other carrier systems [6,7], with the most widely researched being the first two. Examples of external triggers are ultrasound (US), electromagnetic waves (light-triggering) and magnetic fields, while internal triggers include $\mathrm{pH}$, temperature and enzymes [8].

This review focuses on liposomes as the drug carrier and US as a triggering technique and also includes a brief review on the history of triggering techniques. Section 2 thoroughly discusses the liposomal carriers including a description of their structure, their advantages and disadvantages. Section 3 reviews the different triggering techniques, both external and internal, with a brief description of the advantages and disadvantages of each. Section 4 deals with the use of US to trigger drug release from liposomes, mentioning the physics of US, how US can interact with liposomes to release the drug, advantages and disadvantages on the use of US, relevant in vivo research, and clinical uses.

\section{LIPOSOMES}

\subsection{Introduction}

A full DDS is composed of two main parts. The first part is related to the carrier vehicles that circulate in the body, and then deliver their contents to the desired location. These carriers include liposomes, micelles, dendrimers, archaeosomes, solid lipid nanoparticles and others [9-11], but in this work, only liposomes are discussed. The second part of the DDS is the controlled release of the drug from the nanocarriers using triggering methods [8].

To improve the utility of these nanovehicles, so they can circulate in the blood stream and accumulate at the desired location, targeting techniques are used to modify their surface so that they can sense their way in the body and accumulate preferentially at the tumor site. There are two broad types of targeting techniques, namely passive and active (ligand) $[5,8$,
12, 13]. In passive targeting, the vesicles accumulate preferentially at the tumor site based on several malignancy physical parameters, including $\mathrm{pH}$ level, capillary size, enzymatic concentration, and leaky vasculatures [14]. Additionally, solid tumors have damaged lymphatic drainage systems. Together, these factors promote the extravasation and accumulation of macromolecules and nanoparticles at the tumor site, a phenomenon termed the enhanced permeability and retention effect (EPR) $[15,16]$. This type of targeting requires longer circulation periods allowing for the carrier to accumulate at the targeted location before release occurs $[5,8]$ (see Section2.3. Liposome stability - stealth liposomes). Furthermore, in passive targeting, the carriers can also be designed to respond to the intended trigger. Hence, the physical environment in which the drug is introduced can be used both as a targeting parameter and as a triggering technique, as will be discussed later. Nonetheless, while controlling the release using physical parameters may prove challenging, it may be a desirable feature in case most of the loaded drug dosage contained in the carriers is to be completely released $[5,9,12,17,18]$.

The nanocarriers can also be designed in such a way that they have higher affinity towards certain cancer cells, when compared to their affinity towards healthy cells. This is done using ligands that can biochemically recognize certain features of cancerous cells (namely receptors), thus allowing them to specifically bind to those cells. This type of targeting is termed active targeting. Active targeting can be further divided into three levels: first-order, second-order, and thirdorder targeting [13]. The first-order targeting usually refers to organ targeting, where the carriers are programmed to accumulate in a certain organ and release the drug in the desired region. This is the lowest form of active targeting as it is not sufficiently selective. The second-order targeting, cell targeting, is more selective as the carriers bind to the surface of the cells inside the organ where the drug is released. This form of targeting is more focused, yet not very selective because the drug is released over the whole region, including healthy cells in the vicinity of the cancer tissue. The third and most selective active targeting form is called the subcellular targeting. The carriers used in this form have the ability to select specific cells, enter their cytosol, and release their contents afterwards [19, 20]. Using the thirdorder targeting, only cancerous cells are treated with the chemotherapy, while the adjacent healthy cells are usually spared the cytotoxicity of the anti-neoplastic agent $[12,13,18]$.

\subsection{What are Liposomes?}


In the early 1960s, Alec Bangham, a British scientist, was conducting experiments with phospholipids when he recognized spherical structures developing when water was added to the phospholipid film. Later, he discovered that those spherical structures, known as liposomes, were hollow and could encapsulate chemicals [17, 21, 22]. Liposomes are drug delivery vehicles in the nanosize range which can be used to encapsulate chemotherapeutic drugs until reaching the location of diseased tissues inside the body. They are comprised of phospholipid bilayers similar to cell membranes (Fig. 1), which is an advantage when compared to other nanoparticles. from the MLVs by physical means, e.g. high intensity focused US (HIFU). These ULVs can be further classified into three types: small unilamellar vesicles (SUVs), large unilamellar vesicles (LUVs), and grand or giant unilamellar vesicles (GUVs) [27] (Fig. 2). The most appropriate vesicles for drug delivery are the ULVs due to their average size and their ability to encapsulate all types of drugs [24].

Other features of liposomes, including thermotropic behavior, phase transition temperature (or temperature range), and maximum transition temperature $\left(\mathrm{T}_{\mathrm{m}}\right)$, are also determined by the lipid composition and their packing parameters. These

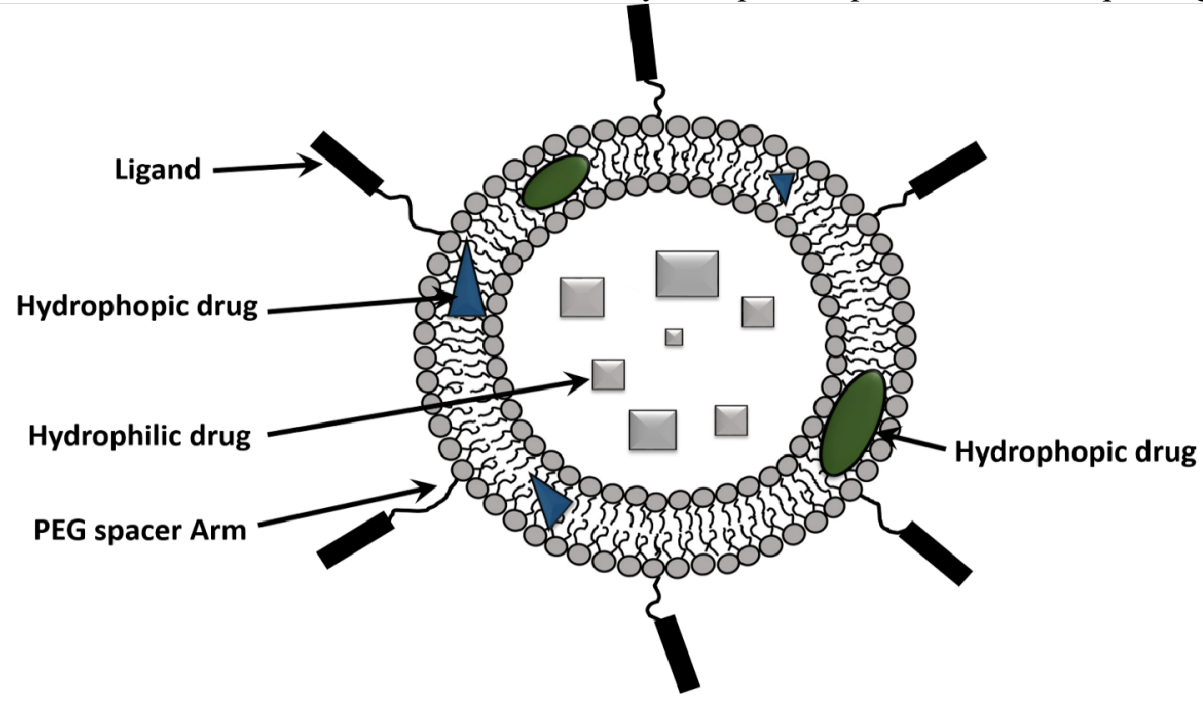

Fig. (1). The general structure of a PEGylated liposome. The figure shows the liposome loaded with a hydrophilic drug in its core, while also carrying hydrophobic drugs in the phospholipid bilayer. The surface is decorated with PEG chains to which a ligand is attached.

Phospholipids are molecules that have two parts, a hydrophilic head and a hydrophobic tail. This composition allows for both hydrophobic and hydrophilic drug encapsulation by the liposomes. Hydrophobic drugs can be loaded between the lipid layers where no water is present, while hydrophilic agents can be loaded in the aqueous core of the liposomal structure. Additionally, liposomes have the advantage that chemical flags (ligands) can be attached to their surfaces to achieve more specific active targeting [21, 23].

The lipids that form liposomes have certain features that determine the structure of the resultant carrier. Those features include the packing parameter which is the ratio of the crosssectional areas of the hydrophobic to the hydrophilic regions of the lipid and can be calculated as follows [17, 24],

$$
P P=\frac{V}{L * A}
$$

where $\mathrm{V}$ and $\mathrm{L}$ are the volume and the length of the hydrophobic part while A refers to the area of the hydrophilic part. In order to form a functional liposome, the packing parameter needs to be between 0.74 and 1.0 [25]. If this threshold is met, using the right technique, full liposomes called multilamellar vesicles (MLVs), can be formed [26]. The MLV are relatively big in size and their main advantage is that they are stable. Unilamellar vesicles (ULVs) can be produced features determine the permeability of the liposomes and their sensitivity to temperature. When liposomes are formed, the lipid bilayer goes through a twophase transition stage. The lipids can change from a solidordered state (SO), sometimes referred to as the gel phase, where each element is discrete and not attached to other elements in a harmonious way forming a crystalline structure, to a liquid-ordered state (LO) where the layer becomes more flexible and homogenous. When the lipids forming the liposomal membrane are all in the SO state or in the LO state, the liposomes are considered to be tight, allowing almost no drug release. However, as the lipid transitions from one state to another, at some point, some of the lipids will be in the SO state while others will be in the LO states. When this happens, in a state referred to as liquiddisorder (LD), the lipids are arranged in such a way that allows the formation of pores in the shell through which the drug starts to diffuse out, i.e., release begins. This transition happens due to a temperature change that leads to the solidification or liquefaction of the lipids. This phenomenon occurs at the $T_{\mathrm{m}}$, during which the maximum change in heat capacity happens and most of the thermal energy is used to re-organize the lipids. This transition temperature is determined based on the packing parameter and the lipid composition of the liposomes. Similarly, other liposomal features depend entirely on the lipid composition hence reasserting the importance of the chemical formulations of those nanocarriers $[17,24,28]$. 
The fact that the liposomal membrane is similar to that of human cells gives them the advantage of being more easily accepted by the human body. Nonetheless, they can still be attacked by the macrophages that patrol the blood constantly, which can identify them as dead cells or foreign structures, thus contributing to their elimination. At the same time, this fact can actually be advantageous in some cases. Some diseases, such as systematic fungal infection and leishmaniase, are due to the infection of macrophages, hence they can be treated by delivering liposome-encapsulated toxic drugs directly into the mononuclear phagocyte system [23]. To

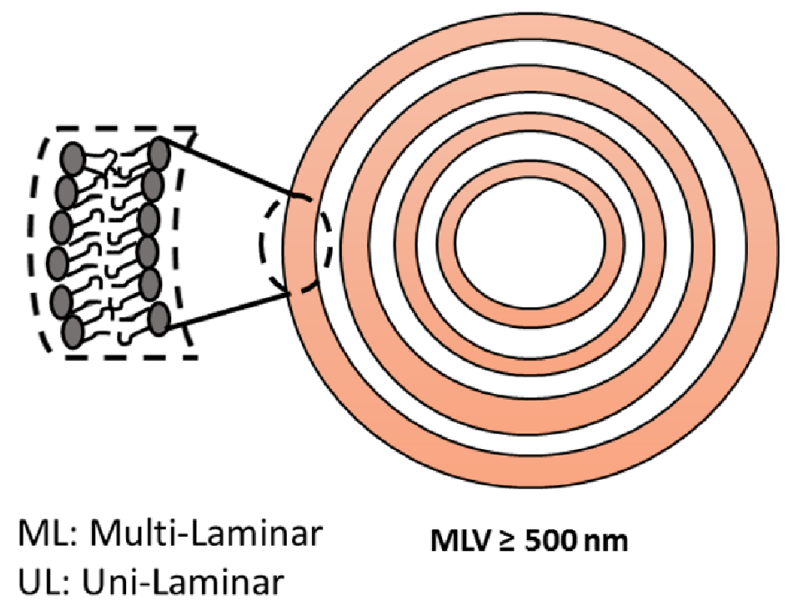

[17]. Both unsaturated and saturated hydrocarbon chains can suffer oxidative processes, although the latter only occurs at high temperatures. In the presence of oxygen, this process, termed lipoperoxidation, develops fast and may lead to the rupture of the hydrocarbon chains. Such reactions cause deformations in the surface of the liposomes that lead to their destabilization. The chemical stability of the liposomes determines their shelf-life and can be enhanced by the addition of antioxidants and chelators of metal ions [30].

Another chemical process that jeopardizes the stability of liposomes is hydrolysis. This process can affect both the

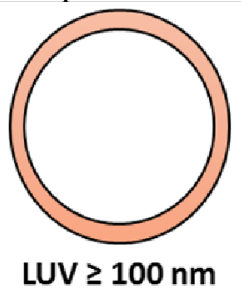

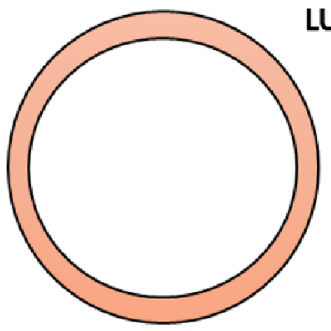

GUV $\geq \mathbf{3 0 0 ~} \mathbf{n m}$

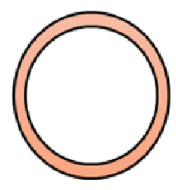

$20 \leq \mathrm{SUV} \leq 100 \mathrm{~nm}$

Fig. (2). Formation of liposomes of different sizes and levels. $L M V$, multilamellar vesicle; $S U V$, small unilamellar vesicle; $L U V$, large unilamellar vesicle; $G U V$, giant unilamellar vesicle. The numbers indicate the size range for each type of liposome.

treat other diseases, a new strategy had to be devised, and this issue was resolved with the synthesis of a new type of liposome called stealth liposomes [23, 29-31].

\subsection{Liposome Stability-Stealth Liposomes}

A crucial characteristic that needs to be addressed when dealing with drug nanocarriers is their stability. Stability is essential since non-stable carriers will prematurely release their toxic content once the patient receives the medication.

Stealth liposomes are high-stability, long-circulating liposomes [23, 29-31]. The inclusion of saturated phospholipids and cholesterol in the composition of liposomes increases their stability, but does not prevent their binding to proteins. To increase the blood circulation time of liposomes, these vehicles are usually coated with a layer of a synthetic polymer called polyethylene glycol (PEG) (Fig. 1), which protects them from binding serum proteins, and thus from being recognized by the mononuclear phagocyte system.

The stability of a liposome is mainly dependent on three factors: chemical composition, colloidal and biological stability, all of which are inter-related $[17,30]$.

\subsubsection{Chemical Formula Stability}

Since liposomes are nano-sized carriers made of lipids, their chemical stability is highly dependent on the stability of these compounds, which are prone to oxidation and hydrolysis carboxylic esters, as well as the phosphate esters that are present in the liposomal structure. The hydrocarbon chains may be completely hydrolyzed leading to the formation of glycerophosphoric acid [17].

To increase the physicochemical stability of the liposomes, cholesterol units are added to the formulation, which, along with the presence of phospholipids containing saturated hydrocarbon chains, make them stiffer and less vulnerable to physical factors [8]. The use of cholesterol needs to be optimized so that the produced liposomes are stable enough to circulate for longer periods in the body, yet their breakdown is possible so that drug release can be achieved at the diseased location. It has been observed that liposomes with cholesterol in their composition have longer blood circulation time, which proves that chemical stability affects biological activity [31].

\subsubsection{Colloidal Stability}

Colloidal stability refers to the stability in the case of collisions between different liposomes and other components of the blood (including blood proteins). When liposomes are systemically introduced into the body, it is inevitable that they will collide with other particles. Those collisions may lead to their disruption and shear, allowing for the release of their contents. To avoid the occurrence of this disruption due to collisions, a surface chemical chain is attached to the surface of the liposomes through either grafting or absorption. The most widely used chemical chain is, as mentioned before, the 
PEG polymer, which is added to the formulation to generate sterically stabilized liposomes (SSL) (the stealth liposomes mentioned previously) [32]. The PEG polymer is a hydrophilic chain that is spread over the surface of liposomes. These chains are capable of shielding the liposomes from colliding with other particles in the body using a repulsive force due to the compression of the chains between the two surfaces. Simply stated, those chains work as springs that create an opposing force when compressed to retain their original length (Fig. 3). These polymers also protect the liposomes against the mononuclear phagocyte become more "visible" to the receptors, which reduces steric interference when binding to the target [28].

2.4. eLiposomes eLiposomes or emulsion liposomes are a new class of stealth liposomes, containing nanoemulsion droplets, which makes them more sensitive to ultrasonic triggering (i.e. more echogenic) [35-39]. This feature is an example of the type of modifications that the liposomes can undergo to improve their effectiveness as drug carriers. Once emulsions are vaporized, the size of the carrier increases by 125 -fold which bursts itopen, thus spilling its contents at the desired
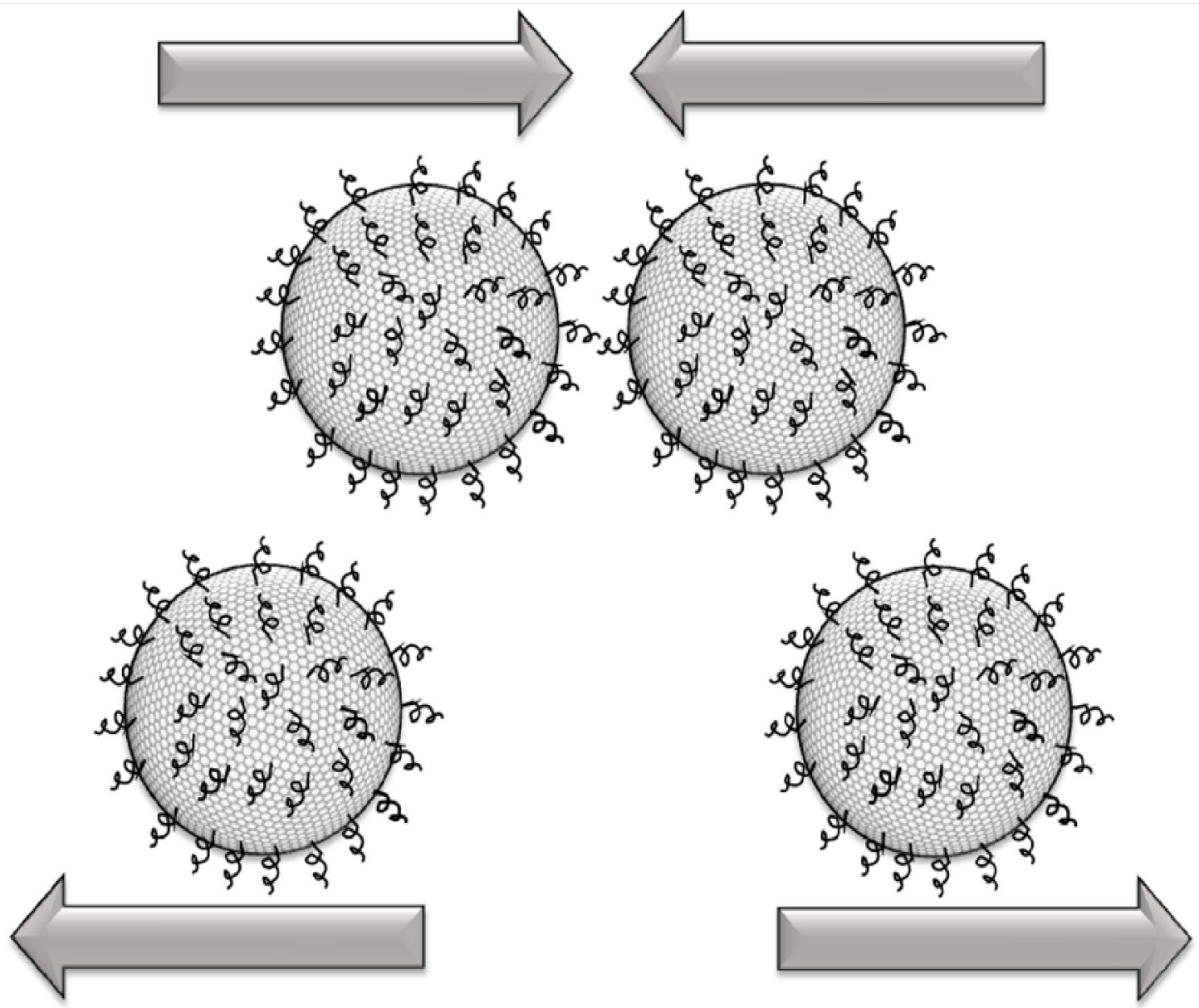

Fig. (3). Stability of PEGylated liposomes. Collisions are prevented by adding PEG chains to the surface of liposomes, which act as springs during collisions.

system, lowering the possibility of the liposomes being ingested by the macrophages, as described earlier. Chain compression is entropically unfavorable allowing for the carriers to circulate in the blood for longer times [33].

However, the presence of these chains on the surface of the liposomes may pose a problem when the liposomes are used for active targeting. The ligands for targeting are usually shorter than the PEG polymers, which present a challenge to the action of target as it prevents the ligands from binding to the receptors on the surface of the targeted cells [34]. Therefore, to achieve better targeting of PEGcoated liposomes, the targeting ligand is attached to the surface of the liposome through a PEG spacer arm. This allows the ligands to be extended further outside the dense PEG-coated surface and location. The emulsions can also act as bubbles that can cause cavitation, which is one of the mechanisms by which drug release from liposomes can be induced (discussed in section 4). However, here, only the structure of the eLiposomes will be discussed.

The main advantage of eLiposomes over other liposomes is the fact that they are more sensitive to US hence release can be induced using low intensity waves [36, 38]. Liposomes are not inherently sensitive to acoustic waves [40]. However, when air bubbles are present in the vicinity, release can occur. This is due to the fact that these bubbles are affected by USinduced cavitation (Section 4.2.2), which leads to the induction of a massive force that causes the liposomes to shear open and release their content. This discovery inspired research on acoustically sensitive liposomes. Additionally, eLiposomes are smaller in size than normal microbubbles which is an 
advantage when considering the leakage (via the EPR effect) in the vascular network of tumors [35]. Their size, also, makes them suitable for ligand-stimulated uptake into cells through endocytosis [37].

In the first attempts to use US to trigger the release from liposomes, the microbubbles, considered as US contrast agents, were introduced in the solution with the liposomes. However, in order to trigger their release, the bubbles had to be of a certain size and composition. It was challenging to deliver them to the targeted site with the liposomes, as they were bigger in size and were easily eliminated by the pulmonary pressure, which introduced various constraints when the system reached the clinical phase [41]. As a solution to this problem, it was then suggested to introduce nanoemulsions into the liposomal structure, to make them more echogenic [37]. Those nanoemulsions are usually made of perfluorocarbons (inert), the two most common being perfluorohexane $\left(\mathrm{PFC}_{6}\right)$ and perfluoropentane $\left(\mathrm{PFC}_{5}\right)$, both possessing low vapor pressures $[35,37]$. The main advantage of these perfluorocarbons is their non-toxic nature (they are currently being investigated as blood substitutes) and their stability in aqueous solutions. They also have relatively low vapor pressures, which make them sensitive to the pressure waves induced by the acoustic field $[36,38]$.

Vapor can be compressed by increasing the external pressure, which forces its atoms to come close together. However, if the external pressure is reduced, the vapor will expand to regain its normal atomic spacing. The emulsions are designed to contain the perfluorocarbon under a certain pressure level at a certain temperature in the form of a liquid. When the external pressure is forced below the vapor pressure, the emulsion will expand and the perfluorocarbon will change from liquid to gas, and since the emulsions are loaded inside the liposomes, they will in turn expand. This expansion leads to the formation of pores in the membrane of the liposomes (and are capable of bursting the liposome open), which causes the release of the drug [39] (Fig. 4).The size of the nanoemulsions has been described to be as small as $30 \mathrm{~nm}$ [37] and as large as $500 \mathrm{~nm}$ [38]. As mentioned above, when the US is in the rarefaction phase (low pressure cycle), the pressure will be below the vaporization pressure of the perfluorocarbon, forcing it to vaporize, leading to the expansion or even the complete destruction of some nanoemulsions. Hence,
eLiposomes are considered useful carriers when US is used as a trigger. Several research studies showed that, when using US as a trigger, drug release from eLiposomes increases, when compared with the release from "normal" liposomes $[35,36$, 38].

\section{TRIGGERING TECHNIQUES}

So far, this review focused mainly on the first part of the drug delivery system, i.e., the carrier. Although this is a crucial part of the system, stimuli or techniques controlling the release of the therapeutic agents from the carriers are equally important. This form of targeting is referred to as the triggered targeting. The objective of a DDS is to deliver the drug to the desired location in the body and to control the release of its content, thus minimizing the side effects of the drug. This section focuses on the different techniques used to trigger the release from the nanocarriers, and in particular from liposomes. There are two types of triggering means: internal triggers including the $\mathrm{pH}$ level, temperature, and time, and external triggers including light, electromagnetic waves, magnetic fields and US [42] (5). Since US is the main focus of this review, section 4 will be dedicated to this triggering technique. It is important to note that some external triggers either directly affect the carriers or are used to induce an internal trigger. For example, electromagnetic waves can be used to directly trigger release or they can be used to heat up the area and cause release through a temperature increase (an internal trigger) [43].

Triggering is defined as the method by which the release of drug is controlled, and it includes the period of release, the amount to be released and the location of the release. Efficient DDS can be obtained by targeting the carriers, and encapsulating a chemotherapeutic agent,until it reaches the cancer cells, followed by its triggered release at the desired site, thus increasing the efficacy of the therapeutic [42]. Each triggering technique is used along with a specific type of carrier that is designed to respond to it. For example, magnetoliposomes are easily triggered by magnetic fields because their chemical composition includes active agents that are sensitive to these external fields, namely superparamagnetic elements including iron [44]. Similarly, other 


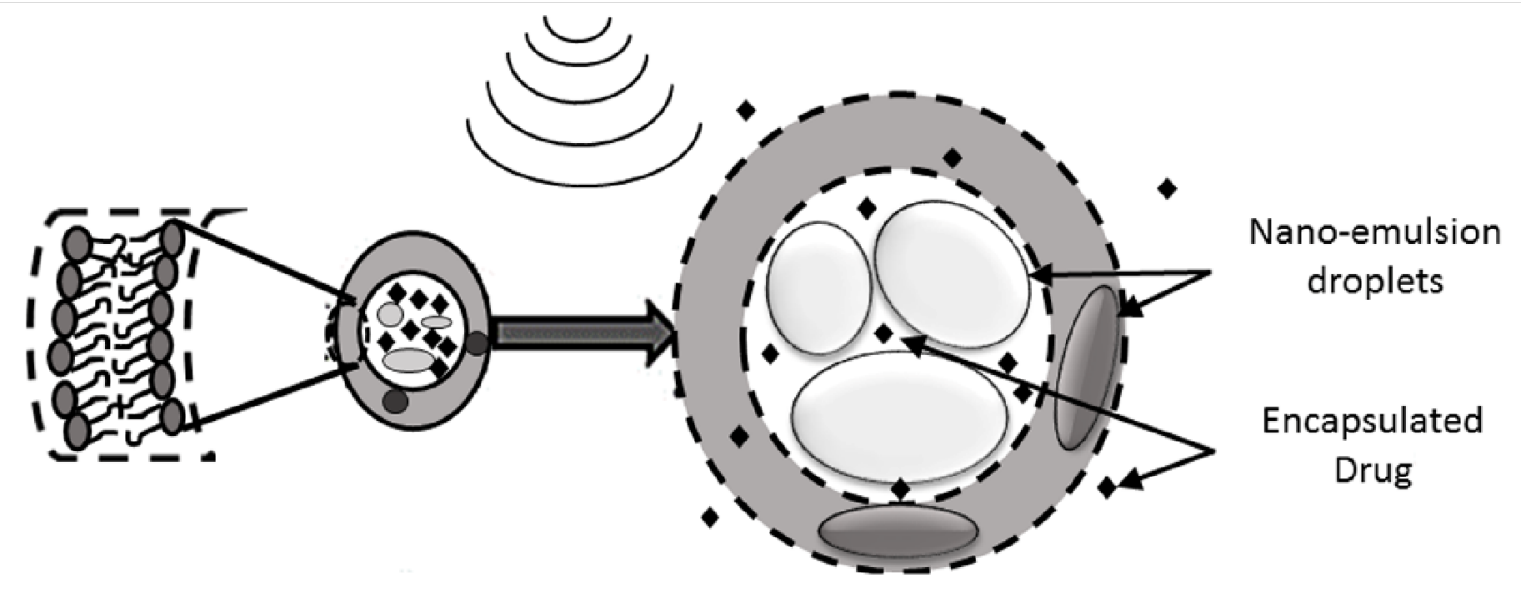

Before Sonication

After Sonication

Fig. (4). Ultrasound-triggered release from eLiposomes. Ultrasound affects the encapsulated nanoemulsions causing them to expand during the low pressure cycle, leading to the expansion and eventually the disruption of the liposomal lipid bilayer.

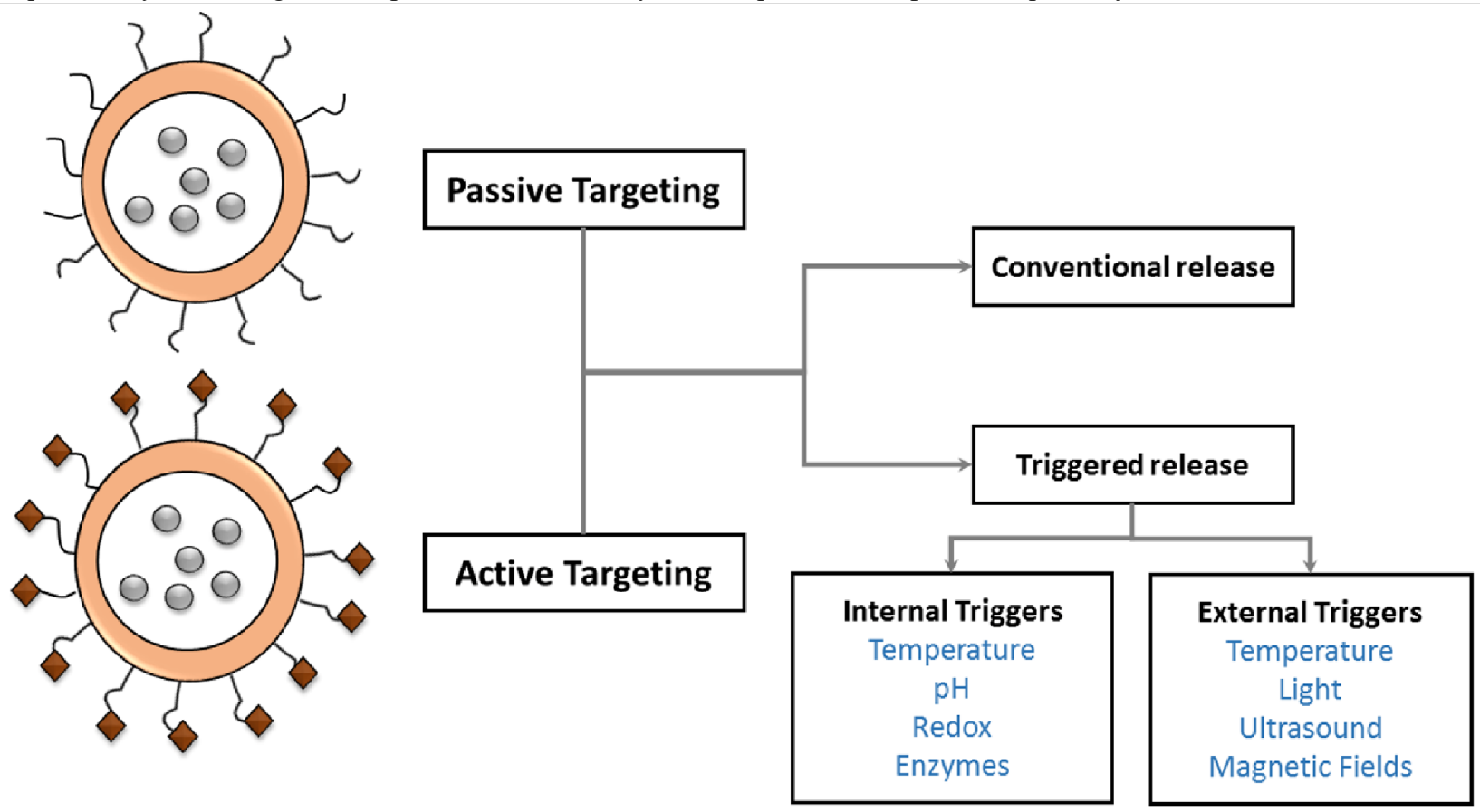

Fig. (5). The two major triggering techniques. Internal triggers and external triggers are used to control drug release from different 
liposomal carriers modified to be sensitive to a specific trigger.

types of nanocarriers have specific agents in their chemical composition that make them sensitive to a certain triggering signal [37].

\subsection{Triggering Methods}

\subsubsection{Temperature-Triggered Release-Thermosensitive Liposomes}

This type of trigger is the most widely used to release drugs encapsulated in liposomes, and it has been extensively reviewed in the last few years [45-49]. Since most triggering techniques revolve around the concept of thermal release, extensive research has been directed towards developing

liposomes that are thermally sensitive. Liposomes are made of lipids that have a thermal threshold above which they start to melt. This causes the surface of the liposomes to become porous, allowing the encapsulated drug to be released. Nonetheless, not all liposomes behave in this manner. Thermally triggered liposomes have a defined chemical composition that makes them vulnerable to temperature, hence they are termed Temperature Sensitive Liposomes (TSL) or thermosensitive liposomes [49-54]. As the name suggests, TSLs are sensitive to an increase in the local temperature, a process known as hyperthermia, which can be induced via external triggering techniques including US, magnetic field, microwave or infrared light [54-57]. If the temperature increase is adequate, so that the temperature reached is higher than the thermal point of the liposomes, it will cause release of the drug within the targeted at the desired location, hence the side effects due to the toxicity of the chemotherapeutic agent are decreased. Usually, the designed TSLs have a thermal point in the range of $39-43^{\circ} \mathrm{C}$ depending on the type of lipids used in the synthesis $[49,58]$. This temperature range is higher than the temperature of the human body $\left(37^{\circ} \mathrm{C}\right.$ on average), which makes TSLs desirable drug carriers, since they are perfectly stable in the body and will not release their contents unless mild hyperthermia is induced.

TSLs were first reported in 1978 by Yatvin et al. [59] and tested in an in vitro model. The original formulation contained 1,2-dipalmitoyl-sn-glycero-3-phosphocholine (DPPC) and 1,2-distearoyl-sn-glycero-3-phosphatidylcholine (DSPC). However, with this formulation, release was not specific and occurred everywhere the temperature of the tissues exceeded a certain threshold, hence further research was done to design thermosensitive targeted liposomes, i.e. TSLs that circulate in the body, and are aimed towards a targeted destination. To accomplish this, liposomes were first stabilized by adding extra saturated fats and cholesterol to their composition [60]. Also, to increase their circulation time in the body, hydrophilic polymers or glycolipids, such as PEG or monosialoganglioside $\mathrm{G}_{\mathrm{M} 1}$, were added to the composition, yielding stealth TSLs [61]. These hydrophilic polymers act as a shield against blood plasma and macrophages, avoiding elimination processes including phagocytosis and endocytosis, hence increasing the circulation time of the carrier in the blood stream. By adding these two extra components, functional triggered TSL liposomes were produced. Further improvements were achieved with the incorporation of lysolipids and temperaturesensitive polymers. Actually, the first TSLs that had the potential to be used in clinics were described in 2000 by Needham and coworkers [62], and were composed of DPPC and $\mathrm{DSPEPEG}_{2000}$, modified with lyso-phosphatidylcholine (lyso-PC).

Thermosensitive liposomes may be divided into three broad groups: traditional TSL (TTSL), lysolipid TSL (LTSL) and polymer-modified TSL (PTSL) [49].

TTSLs make use of the fact that all lipids have a transition temperature, hence the liposomal lipid bilayer undergoes phase transitions according to the temperature, as described in 2.2. Liposomes can be designed to melt at a certain temperature, by carefully planning their formulation [8]. However, it is possible to modify the membrane of the liposomes to make them more sensitive to temperature by adding different molecules to their formulation such as in the case of LTSL which are lysolipid-modified. Lysolipids are lipids that contain only one, not two, hydrocarbon chains, which makes them more prone to form highly curved form of micelles resulting in stabilized defects in the membrane when the $T_{m}$ is approached [49]. One example of a lysolipid is 1myristoyl-2-palmitoyl-sn-glycero-3-phosphocholine (MPPC), which has been combined with DPPC to create liposomes used in in vitro and in vivo studies showing promising results [49].

On the other hand, PTSLs are liposomes with a formulation that includes natural orsynthetic thermosensitive polymers [63, 64]. These polymers have a specific lower critical solution temperature (LCST), above which the polymer changes phase, transitioning from hydrophilic to hydrophobic [8, 49, 58]. When liposomes coated with these polymers are subjected to temperatures above the LCST, the phase change of the polymer chains makes them contract, destabilizing the liposomal lipid bilayer, and making these regions leaky, thus causing the release of the liposomal drug load $[63,64]$. There are several groups of polymers, based on the chemistry of their groups, but the most widely studied is poly(N-isopropylacrylamide) (poly(NIPAM)), which has a temperature threshold of $32^{\circ} \mathrm{C}$ [65]. Poloxamers such as Pluronic $^{\circledR}$, well known in the field of polymeric micelles, have also been used as temperature sensitizers in liposomes.

The first studies using poly(NIPAM) and derivatives [6668] demonstrated that it was possible to anchor these polymers to the surface of liposomes, enhancing their thermosensitivity. The combination of poly(NIPAM) with different co-monomers changes its basic LCST, e.g., the transition temperature of the copolymer of Nisopropylacrylamide and octadecylamine (poly(NIPAMcoODA)) is $29^{\circ} \mathrm{C}$, while that of the copolymer of Nisopropylacrylamide, acrylic acid and octadecylamine (poly(NIPAM-co-AA-co-ODA)) was determined to be $37^{\circ} \mathrm{C}$ [69]. These studies also revealed that the sensitivity to temperature was highly dependent on the liposomal composition, since liposomes with PC showed low release, 
while when the temperature-sensitive DPPC was incorporated into the liposomes, the release significantly increased [66]. Further studies showed that the release could be further improved when 1,2-dioleoyl-sn-glycero3 phosphoethanolamine (DOPE) was used $[68,69]$. The main advantage of DOPE carriers is their unique hexagonal II shape in physiological conditions, forming inverted micelles instead of stable bilayers. This destabilizing effect can be balanced by combining them with the temperature sensitive polymers, creating stable TSLs when the temperature is below the LCST of the polymers. Above the LCST, the polymer chains collapse and release from these TSLs can be triggered prematurely [63]. In any case, liposomes conjugated with these polymers are TSLs with LCST below the physiological temperature of the human body, preventing their clinical use as drug carriers [49, 63]. For in vivo drug delivery, the liposomes must show release at temperatures slightly higher than the physiological. Kono et al. [64] used a combination of poly(NIPAM) with poly(acryloylpyrrolidine) (poly(APr)), a polymer with a LCST around $50^{\circ} \mathrm{C}$, to produce a polymer with a LCST of $\sim 40^{\circ} \mathrm{C}$. Calcein-containing DOPEliposomes, stabilized by the above mentioned copolymers, were then tested for temperature-triggered release. The results confirmed the sensitivity of the liposomes to changes in the temperature, mainly due to the structural change that the copolymers undergo, which causes the membrane to be leaky and allow for the release of the loaded drug. Release was measured using the fluorescence emission over time technique, at temperatures lower and higher than the copolymers' LCST. Almost no release was observed at temperatures below the LCST, but as the temperature reached and exceeded the LCST, significant release was measured. Additionally the authors reported that the biodistribution of these liposomes could be potentially controlled by the presence of the copolymers [64, 67]. In fact, above the LCST, liposomes covered with the copolymer tend to aggregate, since their surface becomes hydrophobic. If present below the LCST, however, the polymer grafts will act similar to PEG, reducing the interaction between the liposome and blood proteins, which can increase their circulation time.

Simultaneously, Nishita and co-workers [70], reported a study using TSL containing cisplatin, triggered with localized hyperthermia, used to treat murine tumor. The results showed that cisplatin was more effective when combined with TSL possessing a LCST of approximately $42^{\circ} \mathrm{C}$. It was observed that the cisplatin effect was enhanced by temperature, as the concentration inside the tumor, within 30 minutes of administration, was 3.4 times higher than when normal liposomes were used. Also, heating the tumor site resulted in an increased retention of the drug which in turn enhanced its effect. This study highlighted the importance of this type of liposomes and encouraged further research in this field.

Later, Paasonen and co-workers [71] used poly $(\mathrm{N}-$ (2hydroxypropyl)methacrylamide mono/dilactate) (pHPMA mono/dilactate) polymer, with a LCST of $42^{\circ} \mathrm{C}$ and studied the effect of its incorporation on TSLs loaded with the fluorescent model drug calcein. Liposomes coated with the polymer showed enhanced calcein release at $43^{\circ} \mathrm{C}$, when compared with the non-coated ones. This enhanced release could be possibly attributed to the precipitation of the polymer, which leads to the liposome aggregation, with subsequent destabilization of the bilayer.

A very important factor to consider when using TSLs is the choice of the proper triggering techniques capable of causing an increase in the surrounding temperature, thus causing the drug release from these nanocarriers. As mentioned earlier, there are internal and external triggering techniques that can be used to induce release from liposomes. Until now, the TSLs that have been described in this section were designed to tolerate physiological temperatures until they arrived at the tumor site and released their contents. Nonetheless, this presents a challenge because the difference in temperature between the tumor sites and the rest of the human body may not be significantly different. Thus the idea of externally triggering the release from TSLs was considered as a possible solution to this problem. This was done by designing TSLs with thermal thresholds higher than the body temperature, usually in the range of $39-45^{\circ} \mathrm{C}$ [46].

One of the first external heating means used in research were microwaves: electromagnetic waves with a frequency range of 0.3 to $300 \mathrm{GHz}$, with relatively short wavelengths [72]. Microwaves are capable of heating by interacting with particles, especially in dielectric material, causing energy absorption and subsequent conversion to thermal energy. In fact, the thermal energy is converted from a mechanical electrical interaction between the fast alternating electric field created by the microwave, and the rotation of the dipole of the atom. This energy conversion is referred to as dielectric heating [73]. The frequencies dedicated to achieve local heating using microwaves are $915 \mathrm{MHz}$, for industrial use, and $2.45 \mathrm{GHz}$ for domestic use [74]. At these frequencies, liquids, especially water, undergo extensive dielectric heating. This concept forms the basis of the function of the microwave ovens used nowadays, and easily explains why dry food is hardly heated by microwaves, while the heating of wet food is easily achieved. The same concept can be used to trigger release of agents from TSLs by locally heating the tumor region using externally applied microwaves [75]. Heating the human body with microwaves is easily achievable since the body mostly consists of water. The TSL accumulate at the tumor site through the EPR effect or by active targeting and then the temperature can be raised using focused microwaves, which leads to localized release. Nonetheless, microwaves penetration is constrained by the skin depth, which is defined as the distance that the microwave can penetrate in the body until all its energy is absorbed and converted into heat. The skin depth $\delta$ is approximated by equation (2) $[76,77]$,

$$
\begin{aligned}
& \delta=\operatorname{sqrt}\left(\frac{2 * p}{\mu_{\mathrm{o}} * \mu_{\mathrm{r}} * \mathrm{w}}\right)=\operatorname{sqrt}\left(\frac{p}{\mu_{\mathrm{o}}^{*} \mu_{\mathrm{r}} * \pi * f}\right) \\
& =\operatorname{sqrt}\left(\frac{1}{\mu_{\mathrm{o}} * \mu_{\mathrm{r}} * \pi * f * \sigma}\right)
\end{aligned}
$$


where $\rho$ is the resistivity of the conductor, $\sigma$ is the conductance of the conductor $(\rho=1 / \sigma), \omega$ is the angular frequency of the field $\left(=2 \pi \times\right.$ frequency), $\mu_{\mathrm{r}}$ is the relative magnetic permeability of object, $\mu_{\mathrm{o}}$ is the permeability of free space [72]. As seen in the above equation, $\delta$ is inversely proportional to the frequency of the microwave hence, the higher the frequency, the more superficial the heating. On the other hand, $\delta$ is directly proportional to the resistivity of the subjected body, but inversely proportional to its magnetic permeability (equal to the permeability of the free space). Since the resistivity of the human body is low, the penetration is somewhat constrained. Furthermore, since the body is mostly composed of water, it will heat up quickly from the outside to the inside. Consequently, if the targeted tumor site is deep in the body, the skin will overheat when attempting to achieve the required triggering temperature. This presents a challenge when it comes to treating a deep internal tumor, yet it is very effective with surface cancers such as skin cancer. In fact, microwaves are used alongside radiotherapy to induce wholebody hyperthermia that can assist in the elimination of skin cancer or superficial tumors [75].

Another method that can be used to generate local heating is referred to as infrared sauna [78]. Similarly to microwaves, infrared signals are waves that carry energy that can be absorbed by the body, and converted into heat. However, although the infrared is a safer option compared to microwaves, it has limited applications. As the name sauna suggests, the waves cannot be localized, hence it provides an overall heating of the body. Otherwise, infrared saunas are just normal saunas with a more sophisticated heating system than the classic charcoal or electric heaters. Recently, farinfrared saunas were developed [78]. The main advantage of this type of sauna is that it does not heat up the air in the room, but still heats up the body. This feature is appreciated by patients who cannot tolerate normal saunas especially since the treatment requires long exposure times. Similar to microwaves, this method can induce hyperthermia that can lead to a controlled release from TSL. Although these saunas are a safe option and they can be used for the treatment of superficial tumors, the disadvantage of having longer sessions compared to microwave sessions, makes them one of the last resources by which temperature-controlled release can be achieved.

Other techniques that use alternating magnetic fields (AMF) or light signals can also be used to induce an elevated temperature at the tumor site; however, each technique needs a specially modified liposome that is sensitive to the specific triggering. The use of magnetic fields to produce local heating at tumor sites was first introduced in Europe in 1957 by Gilchrist and co-workers [79], who suggested the use of magnetic sensitive materials that can be activated using AMF and produce heat. The idea was then developed and applied to cancer treatment, with magnetic fields being used to heat the tumor cells and cause hyperthermia, usually in the range of 42$45^{\circ} \mathrm{C}$, thus causing their death. Magnetic nanomaterials used for hyperthermia are very diverse, and several have been described, from the well-studied iron oxide-based to metallic nanomaterials based on $\mathrm{Mn}, \mathrm{Fe}, \mathrm{Co}, \mathrm{Ni}, \mathrm{Zn}, \mathrm{Mg}$ and their oxides [48]. When the TSLs were introduced, magnetic fields were considered as a method by which local heating could be achieved from an external source, thus achieving controlled release. The practice was to introduce targeted TSL that contain the therapeutic agents through normal means such as injections or tablets. On the other hand, the ferromagnetic elements had to be delivered with the highest accuracy possible. Unfortunately, this proved rather challenging. In fact, if such accurate delivery was possible, then why not use this delivery technique to deliver the active therapeutic agent directly? Therefore, the idea was abandoned. Additionally, although this technique outperforms the other techniques in terms of safety, it is not the preferred technique and is extremely challenging. Later, with the introduction of magnetic-sensitive liposomes, called magnetoliposomes, the use of magnetic fields as an external thermal triggering technique was further researched [44, 8082] (discussed in 3.1.5).

\subsubsection{Enzyme-Triggered Release}

The main goal of a DDS that utilizes liposomes as the carrier is to break down the lipid bilayer, thus allowing the encapsulated drug to escape into the tissue or even penetrate directly into the cell cytosol. As described, the liposomes are designed to be stable and robust yet sensitive to a certain stimulus that allows for the controlled release of their contents. Hence, it is wise to look at the environment in which the liposomes are introduced and exploit every possible factor that can be used as a trigger. Some of the most important factors that have been investigated are the enzyme concentrations and $\mathrm{pH}$ levels [83].

It is critical to keep in mind that the cellular environment is dynamic and may change if affected by cancer. This can be used as an advantage in case the tumor site environment shows, when compared to healthy body tissues, an elevated level of one of the factors that can be used as a target. For instance, the activity of certain enzymes may be increased solely at the tumor site, so liposomes can be designed to target the site, where drug release can be triggered using those enzymes. This enzyme-triggered release is an internal triggering technique in which the liposomes are programmed to search for the location with elevated enzyme activities and accumulate at that location. Liposomes sensitive to enzymes are called bio-responsive, since they are chemically modified to be affected by a biological trigger. Additionally, they are programmed to initiate release when the enzyme level is above a certain threshold. For this purpose, the enzymes of interest are those that can disturb the liposomal surface and induce release. Usually lipases are used to hydrolyze the phospholipids that compose the liposomes, but cancerassociated proteases can also be used, when the liposomes have stabilizing polymers attached via peptide bonds [84]. Phospholipases are enzymes that catalyze the hydrolysis of phospholipids. Several studies were performed to prove the possibility of designing a successful DDS that is based on liposomes as carriers and phospholipase activity as the trigger (reviewed in [85]). The works of Luk et al. [86] and Nieva et $a l$. [87] showed that phospholipase $\mathrm{C}$ induces the aggregation and fusion of liposomes. However, it was observed that this enzyme activity is not high in tumors. Phospholipase A2 
(PLA2), on the other hand, is upregulated in infectious and inflammatory diseases and high concentrations of this enzyme have been found in tumors [88, 89]. PLA2 enzymes catalyze the hydrolysis of aggregated lipids, but not the hydrolysis of single lipid molecules, hence they are a perfect choice for an enzyme triggered release from liposomes. Additionally, it was observed that the lysolipids and fatty acids that form during the reaction catalyzed by PLA2 act as permeability enhancers, hence increasing the drug transport across the biological membranes [90-92].

PLA2 enzymes are sensitive to surface charge, hence liposomes can be made more sensitive to their action by modifying their surface with lipopolymers, creating what are called LiPlasomes [85]. LiPlasomes are liposomes designed to be susceptible to hydrolysis by PLA2 enzymes, which are composed of uncharged DSPC, anionic DSPG (1,2distearoyl$s n$-glycero-3-phosphatidylglycerol), and the lipopolymer DSPE-PEG2000 (1,2-distearoyl-sn-glycero-3 phosphoethanolamine-N-poly(ethylene glycol)-2000) [85]. This is a good example of enzyme triggered release as the in vitro and in vivo results suggested the feasibility of using such carriers along with chemically modified prodrugs to achieve successful rates of cancer treatment [93, 94]. Unfortunately, the results obtained with cisplatin-LiPlasomes in a phase 1 clinical trial, showed no significant differences between the treatment with free drug and encapsulated drug [95]. However, this approach is promising and further developments of the formulation are being pursued.

Meers and co-workers [96] reported another enzyme activity that can be used as a trigger,having an enhanced fusogenicity when close to cancer cells. Elastases are proteases that catalyze the hydrolysis of elastin, a fiber present in connective tissue. They have specificity for uncharged amino acids, e.g. alanine and valine [97]. Additionally, it is known that cancer, as well as inflammations, are associated with an increase in this enzyme [98]. Hence, liposomes were designed with a targeting moiety composed of small peptides that are sensitive to the action of elastase [96]. These liposomes contain DOPE, which is not stable at the physiological temperature, neutral $\mathrm{pH}$ and average salt concentration. However, since DOPE is zwitterionic under these conditions, when attached to peptide chains, it becomes negatively charged, which increases its stability, making it possible to synthesize stable liposomes that can circulate in the body. When these liposomes reach the vicinity of the tumor site, the peptides are hydrolyzed by elastase, allowing DOPE to return to its zwitterionic form. Additionally, these liposomes are designed to contain trace amounts of positively charged lipids, hence, upon enzymatic cleavage the liposome becomes positively charged. The charge reversal assists in the process of liposomal binding to cell membranes, since most cells are negatively charged. This is the first step that leads to the fusion of the liposomes and the targeted cells, and can occur before or after endocytosis [96]. A similar strategy was described by Davis and Szoka [99] who created alkaline phosphatasesensitive liposomes, with a release mechanism similar to the one described in [96].
Another successful bio-responsive DDS is exemplified by estrogen-modified liposomes that target prostatic carcinomatous tissues, where the concentration of phosphatase is higher than in normal tissues. The liposomes modified and treated with stilboestrol diphosphate, a phosphorylated synthetic estrogen, are targeted towards the prostatic tumor. Once there, the phosphatase can interact with stilboestrol diphosphate causing the liposomal membrane to be sheared and the contents to be released [8].

A different, but alsoan interesting approach, consists on the use of the phospholipids building blocks in the liposomal formulation as a prodrug. The prodrugs released after the degradation of the phospholipids are activated at the tumor site by overexpressed enzymes [93]. For example, it is possible to design prodrugs similar to lipids that can form liposomes, and use lipases to trigger their release at the target site [85].

From what has been described thus far, it is clear how important it is to exploit the cancer region in a search for the best triggering technique to be used, as well as the chemical formulation of the liposomes, which makes them sensitive to a certain trigger. The importance of the chemical formula was emphasized recently, in a review paper by de la Rica and coworkers [84]. Another factor that has been extensively studied when exploiting the tumor environment is the $\mathrm{pH}$ level, which is reviewed next.

\subsection{3. pH-Triggered Release}

As discussed in the previous section, liposomes synthesized using DOPE linked to a peptide chain can be activated by the catalytic action of specific enzymes, namely elastase [96]. A version of these liposomes which contained $\mathrm{pH}$-dependent cationic lipids was designed to be more sensitive to elastase. After elastase activation and the fusion of the liposomes with the cells, the low $\mathrm{pH}$ of the endosomes allowed the destabilization of the liposomes causing the subsequent release of the liposomal contents. This is an example of $\mathrm{pH}$-triggered release from $\mathrm{pH}$-sensitive liposomes (reviewed in [100-103], first described by the group of Yatvin [104]. $\mathrm{pH}$-sensitive liposomes are stable at physiological $\mathrm{pH}$ but, under acidic conditions, they become destabilized and have fusogenic properties, which causes the release of the encapsulated molecules [102]. As for the triggering mechanism, the $\mathrm{pH}$ change can be either caused by a lower $\mathrm{pH}$ level in the environment close to the tumor, or possibly with the help of enzymes, as described in the fusogenic liposomes work presented by Meers [96].

It has been observed that the $\mathrm{pH}$ level around tumor sites is usually more acidic, around 6.5 , than in healthy tissues with an extracellular $\mathrm{pH}$ in the vicinity of 7.4 [83]. Hence, $\mathrm{pH}-$ triggered liposomes could be designed to release their contents once they reach the tumor site. However, the most acidic sites in the tumor are often distant from its microvasculature, and hence, are hardly reached by liposomes. Consequently, the triggering of liposomes was redesigned to occur after endocytosis [105], inside endosomal vesicles, where the $\mathrm{pH}$ is usually lower than 5. This prevents the liposomes from gettinginto the lysosomal phase, where the biological activity of the drug can be decreased or lost by the action of hydrolases 
and peptidases $[100,101,103,106]$. Furthermore, several reports indicate that in these liposomes, the encapsulated drugs are efficiently transferred from the endosomes to the cytoplasm of the target cell $[107,108]$. Several mechanisms may be involved in this intracellular transfer: $\mathrm{pH}$-induced fusion of the liposome and endosome membranes, with direct release into the cytosol; destabilization of endosomal membranes caused by the destabilization of the liposomal membranes, and drug leakage into the cytosol; destabilization of the liposome and release inside the endosome, followed by diffusion or translocation of the molecules to the cytosol [100].

Essentially, there are four classes of $\mathrm{pH}$-sensitive liposomes, in accordance with the mechanism which utilizes $\mathrm{pH}$ as a trigger [100-103].

The first class makes use of polymorphic lipids such as phosphatidylethanolamine (PE) and derivatives (e.g., DOPE), in combination with amphiphilic compounds that contain one acidic group, forming stabilized liposomes at neutral $\mathrm{pH}$ [28]. This class of liposomes and the pHtriggering mechanism has been extensively reviewed. Unlike most phospholipids, PE has a cone shape, due to its small headgroup, which is poorly hydrated [109], and tends to adopt a hexagonal form. At neutral $\mathrm{pH}$, the stabilizing amphiphilic compound is negatively charged and it increases hydration, which stabilizes the lipid in the lamellar form, preventing aggregation and fusion. At acidic $\mathrm{pH}$, however, the amphiphile is protonated, hydration decreases and the PE reverts to the hexagonal phase, thus promoting fusion [110]. However, these are the least efficient $\mathrm{pH}$-sensitive liposomes as they are rapidly cleared and easily destabilized by serum proteins. This is because, to make liposomes more stable so they can circulate for longer periods, modifications, such as the inclusion of cholesterol and PEG or its derivatives which, as discussed in the previous section, has the ability to circumvent the stability issue of these liposomes, has to be added to the PE lipids. Nonetheless, these modifications also make those liposomes less sensitive to $\mathrm{pH}$ changes, hence there is a tradeoff between stability and $\mathrm{pH}-$ sensitivity $[111,112]$.

The second class of $\mathrm{pH}$-sensitive liposomes, named "caged" liposomes are formed from lipid derivatives that are chemically engineered to have $\mathrm{pH}$-sensitive chemical bonds that are altered when the liposomes are subjected to an acidic environment. This leads to the destabilization of the liposomal membrane, which in turn leads to an increased permeability of the encapsulated molecules as well as increased fusogenicity $[100,101]$. This class of liposomes is helpful when the fusion process occurs between the liposomes and the endosomes or lysosomes, as the $\mathrm{pH}$ inside these vesicles is low, thus causing the lipid derivatives to change form, which in turn allows the release of the drug. This class of $\mathrm{pH}$-sensitive liposomes makes use of lipids such as $N$-acylated aminophospholipid derivatives, plasmalogens, and others, as recently reviewed by Ferreira et al. [101]. Compared to the previously described class of liposomes, these nanoparticles displayed higher levels of $\mathrm{pH}$ sensitivity in the presence of serum protein [113].

The third class of $\mathrm{pH}$-sensitive liposomes follows the same triggering process described for the previous class, but uses peptides that are sensitive to acidic environments, or reconstituted fusion proteins, which are added to the liposomal composition, and are capable of destabilizing the membrane at low $\mathrm{pH}$ [114]. These liposomes were developed based on the fact that viruses enter the host cells by binding to receptors overexpressed on their surface, followed by the direct fusion with the membrane (endocytosis). The binding step is mediated by viral glycoproteins, and these were used as a first attempt to create modified liposomes called virosomes, in 1975 [115]. Afterwards several synthetic peptides have been designed to interact with the liposomal bilayer in a $\mathrm{pH}-$ dependent way (reviewed by [100]), to create $\mathrm{pH}$-sensitive liposomes.

The fourth and most developed class in this field incorporates $\mathrm{pH}$-titratable polymers, which are susceptible to conformational changes at low $\mathrm{pH}$, into the liposomal composition, as reviewed by Felber and co-workers [116]. These polymers include the previously mentioned temperaturesensitive poly(NIPAM), poly(alkyl acrylic acid)s, modified poly(glycidol)s, polyphosphazenes and poly(malic acid)s.

When thermosensitive liposomes were developed, it was observed that the combination of poly(NIPAM) with titratable monomers in liposomal systems increased the LCST above $37^{\circ} \mathrm{C}$, as described in section 3.1.1. Simultaneously, it was observed that the liposomes also became $\mathrm{pH}$-sensitive [117, 118]. Acrylic acid, methacrylic acid, propylacrylic acid, and $\mathrm{N}$-glycidylacrylamide are some of these titratable monomers. At the neutral $\mathrm{pH}$ of blood, the carboxylic groups are ionized and the polymer exhibits an extended conformation. Inside endosomes, however, the acidic $\mathrm{pH}$ leads to the protonation of the carboxylic groups, which reduces the copolymer solubility, allowing the hydrophobic interaction with the liposome bilayer, and its consequent destabilization, which makes the liposome leaky [116]. Including PEG in the formulation increases the liposome stability, as mentioned previously, but PEG usually decreases the fusogenicity and $\mathrm{pH}$-sensitivity of the liposomes [103]. However, the group of Leroux [119] described the synthesis of serum-stable $\mathrm{pH}$-sensitive liposomes, by inserting a modified NIPAM/methacrylic acid copolymer ((poly(NIPAM-co-MAA)) in the lipid bilayer of PEG-stabilized liposomes. The enhanced release of fluorescent markers or Dox from PC and PC/cholesterol liposomes at $37^{\circ} \mathrm{C}$ in acidic conditions similar to those found in vivo suggested the possibility of creating pHsensitive liposomes using those copolymers, which can withstand the human body conditions and successfully deliver the drugs to the tumor cells $[100,103]$. Interestingly, it has also been observed that the inclusion of PEG on the liposome surface did not affect the contents release in an acidic environment indicating that these $\mathrm{pH}$-sensitive liposomes can be stable in blood circulation. Unfortunately, there are not enough in vivo studies using these liposomes to allow the prediction of their possible clinical uses [116].

Recently, a new type of $\mathrm{pH}$-sensitive liposomes, called fliposomes, has been described [120-124]. These contain amphiphiles such as trans-2-morpholinocyclohexanol that 
undergo a pH-triggered flip, which disrupts the liposomal membrane allowing the release of the encapsulated molecules. The proposed mechanism involves a protonationinduced conformational change, and was described by Liu et al. [121]. These liposomes are highly stable in serum and, in weakly acidic medium, exhibit quick release $[120,123,124]$.

In summary, $\mathrm{pH}$-sensitive liposomes are liposomes that were chemically modified, either through the addition of PE agents, $\mathrm{pH}$-sensitive lipids or peptides, or the attachment of titratable polymers, so that they become responsive to a change in $\mathrm{pH}$, which makes their membrane leaky and/or increases fusogenicity, allowing for drug release. Additional information on the different classes of $\mathrm{pH}$ sensitive liposomes and the advantages and disadvantages of each can be found in the review by Drummond et al. [100].

$\mathrm{pH}$-Sensitive liposomes have been used in vitro and in vivo studies. For example, the groups of Mamasheva et al. and Shi et. al. $[113,125]$ described the use of $\mathrm{pH}$-sensitive liposomes with a folate moiety to target cancer cells in vitro. Several other in vitro studies have been extensively reviewed $[100,101,103,116]$. In vivo studies using this type of liposomes are rare, however $[101,116]$. Carvalho-Junior et al. [126] compared the efficiency of cisplatin administered in free form or encapsulated in stealth $\mathrm{pH}$-sensitive liposomes, for the treatment of solid Ehrlich tumors in a mice model. Cisplatin is very cytotoxic, hence it causes several unwanted side effects. The authors observed a longer circulation of ciplastin in its encapsulated form, which led to a higher concentration of the drug in tumor tissue. Additionally, the retention of cisplatin by renal tissue was lower when using the encapsulated drug, which supports a promising role for $\mathrm{pH}$-sensitive liposomes in the alleviation of the nephrotoxicity cause by cisplatin $[126,127]$. Another study by the same group [128] confirmed that the use of these liposomes allows the usage with higher dose of cisplatin, which leads to a significant decrease of the tumor volume, and increase of the tumor growth inhibition, without increasing the side effects, when compared to non-pH sensitive liposomes and free cisplatin.

Paliwal et al. [129] synthesized pH-sensitive liposomes with an estrogen moiety for targeted delivery of Dox to estrogen-sensitive cancer cells (e.g., MCF-7). Their in vivo studies using female Balb/c mice showed an increased efficiency of these liposomes in the inhibition of tumor growth, when compared to non-pH sensitive liposomes and free Dox. Additionally, the use of these liposomes significantly reduced the cardiotoxicity of Dox, a wellknown side effect of this drug.

The group of Ishida $[130,131]$ studied several different Dox-encapsulating $\mathrm{pH}$-sensitive liposome formulations against human B cell lymphoma using mice xenografted with Namalwa cells. The non-targeted formulations showed no increased efficacy when compared to free Dox, but mice treated with targeted several anti-CD19 (a B-lymphocyte antigen) formulations had an increased lifespan. The lifespan was slightly higher (1.5-fold) for mice treated with the targeted $\mathrm{pH}$-sensitive liposomes when compared to the targeted regular liposomes. This modest result is in contrast with in vitro assays obtained by the same group, and was considered to be due to the fast clearance of the liposomes in the blood.

\subsubsection{Light-Triggered Release}

Light usually refers to the part of the electromagnetic radiation spectrum that can be detected by the human eye. It comprises waves with wavelengths from $400 \mathrm{~nm}$ (violet light) to $700 \mathrm{~nm}$ (red light). Immediately adjacent to the visible light spectrum is ultraviolet (UV) light $(100-400 \mathrm{~nm})$ and the near infrared (IR) light $(650-1000 \mathrm{~nm})$, which are usually also considered forms of light. Since light is a wave, it contains energy that can be converted to other forms of energy.Light presents several advantages that renders it as one important trigger for drug release: pulse duration, intensity, cycle and wavelength can be controlled, the beam can be focused at the targeted location, and a variety of tissues can be irradiated easily and without the need for surgical procedure [132]. Nonetheless, just like with any other type of trigger, the nanocarriers to be used must be modified to become sensitive to the triggering means. The principle(s) of phototriggering include light-induced isomerization or polymerization of photoreactive lipids and photosensitization by membrane anchored hydrophobic probes [133, 134]. In the early 1990s, scientists took interest in developing liposomes that are sensitive to light and there was a breakthrough when they succeeded in creating what is currently known as photosensitive liposomes. Anderson and co-workers [135] were able to chemically modify the liposomal structure to include light sensitive lipids that change form when subjected to light. They were able to achieve a photoactivated drug release, by creating a liposomal formulation with the right concentrations of plasmalogen (1alk-1'-enyl-2-acyl-snglycero-3-phosphocholine) lipids and zinc phtalocyanine $(\mathrm{ZnPc})$, a photosensitizer. The mechanism involved the sensitized photooxidation of the vinyl ether linkage of the plasmalogen, followed by its cleavage, which changed the membrane permeability.

However, there are several biological constraints associated with photoactivated release such as biocompatibility, plasma instability, and near infrared sensitivity [136]. In 1996, a paper on triggerable plasmalogen liposomes was published to introduce a newly developed lightsensitive type of liposomes that were able to avoid the side effects associated with the earlier photoactive drug release processes [136]. The authors synthesized plasmenylcholine liposomes that are sensitive to light with wavelength in the spectrum band of 630 to $820 \mathrm{~nm}$. This range was chosen due to the availability of sensitizers absorbing in this range, the fact that light in this range has a penetration depth of more than 0.8 $\mathrm{cm}$ for any tissue, the stability of the liposomes that can be preserved until they reach the targeted location, and light sources in this band that are available. As in the previous work [135], a light sensitizer was added to the liposome formulation, to make it photosensitive. In this work, the authors used three type of sensitizers: the previously used $\mathrm{ZnPc}$, tin octabutoxyphthalocyanine dichloride $\left(\mathrm{SnCI}_{2} \mathrm{Pc}(\mathrm{OBu})_{8}\right)$, and bacteriochlorophyll $a$ (BChla). The effect of these compounds on the release kinetics was monitored, and the authors 
concluded that the increased release upon irradiation is due to the photooxidative cleavage of the plasmenylcholine vinyl ether bond which is close to the hydrophilic interfacial region. This event is responsible for the liposome aggregation and membrane fusion, leading to the release.

In 1999, Bisby and co-workers [137] reported the synthesis of a new type of light-triggered liposomes. In this work, the authors used a sensitizer called Bis-Azo PC, which is a synthetic phospholipid with acyl chains containing azobenzene moieties. When these lipid molecules were subjected to pulsed UV light, their form changed from the stable E-isomer, sterically compatible with a packed stable lipid bilayer, to the more bulky Z-isomer, which created pores in the membrane, allowing the leakage of the encapsulated drug. Later, the authors also reported that the addition of cholesterol to the composition increased the sensitivity of the liposomes, making it possible to use visible light for the photosensitization [138]. These results suggested the possibility of controlling the release rate through the wavelength-dependent release from cholesterol and non-cholesterol liposomes, by sequential exposure to visible and UV light. In photo- triggered release, the composition of the liposomes is critical to their efficiency of response to the photo triggering process. This importance was highlighted in the work by Yavlovich et al. [139], which described the synthesis of photosensitive liposomes with a photopolymerizable diacetylene phospholipid $\left(\mathrm{DC}_{8,9} \mathrm{PC}\right)(1,2-$ bis (tricosa-10,12-diynoyl)-sn-glycero-3-phosphocholine). Upon exposure to UV light ( $254 \mathrm{~nm}$ ) release of calcein could be observed from DPPC/ $\mathrm{DC}_{8,9} \mathrm{PC}$ liposomes, but not from egg $\mathrm{PC} / \mathrm{DC}_{8,9} \mathrm{PC}$. The release was due to the UV-induced photopolymerization of the $\mathrm{DC}_{8,9} \mathrm{PC}$. Later, the same authors [134] were the first to report the in vitro killing of cancer cell cultures, following light-triggered release of Dox from these photosensitive liposomes. The liposomes contained different concentrations of DPPC, DSPE-PEG2000, egg PC, and $\mathrm{DC}_{8,9} \mathrm{PC}$, and were subjected to UV $(254 \mathrm{~nm})$ or visible light (laser, $514 \mathrm{~nm}$ ). It was successfully documented how differently composed liposomes have different release rate. This discovery ignited the interest in designing liposomes that can be completely controlled in terms of release rate, stability, targeting, selectivity, and circulation time.

Aygun et al. [140] presented a very interesting work where scanning electron microscopy was used to study the photoinduced release from 1,2-dimyristoyl-sn-glycero3phosphocholine (DMPC) or DSPC liposomes stabilized by cholesterol and containing the photosensitizer ZnPC. The authors studied the effect of changing the liposome composition on the encapsulation capacity, morphology and photo-induced release properties of these liposomes. It was observed that the DMPC liposomes were more sensitive to light than the DSPC ones, who showed slower release rates. Both liposomes were more sensitive to visible light in the 400$700 \mathrm{~nm}$ range than to $365-\mathrm{nm}$ UV light. The study also determined the optimal ratio of lipids: cholesterol: $\mathrm{ZnPC}$ (7:2:1) for liposome stability.

In the above described studies, the trigger directly causes a change in the carrier. However it is possible to use a trigger to cause a change in the environment that leads to a change in the carrier, upon which the release is observed. For example, when the trigger is light, its energy can be changed into other forms, e.g. heat. Since thermosensitive liposomes are affected by hyperthermia, researchers investigated the use of materials that can be heated when subjected to light, such as gold. In the work of Paasonen et al. [141], gold nanoparticles were loaded into TSL liposomes or attached to their surfaces, and then irradiated with UV light. It was observed that the UV light specifically triggered the release from these liposomes, while traditional liposomes were unaffected. The hypothesis that the gold particles have the ability to absorb the light energy and change it into heat, which is transferred to the liposomal lipids causing a phase transition, was further studied by Mady and coworkers [142] who later performed the biophysical characterization of these liposomes. If the temperature can be raised high enough to cause the lipid bilayer to undergo phase transition from gel phase to rippled phase and then to fluid phase, then release is achieved. By controlling the amount of the gold particles present and the illumination intensity of the UV light, a controlled release can be achieved. The group of Paasonen also studied the effect of these liposomes in cell cultures [143]. Gold nanoparticle-loaded liposomes encapsulating calcein were UV-triggered after cell internalization and the results showed that the cell viability was not decreased, which suggests that this system can be applied in vivo.

Most of the studies described so far use UV or near-UV light, which poses a problem when extrapolating to potential clinical uses. UV radiation is phototoxic and may affect the stability of biological systems, for example by generating reactive oxygen species. In this sense, the use of infra-red (IR) or near-IR to trigger release from photosensitive liposomes, would provide a safer method. Additionally, IR or near-IR light has a deeper penetration into tissues [144]. Recently, Carter and co-workers [145] described liposomes containing porphyrin-phospholipid as a photosensitizer, characterized them, and studied their effect in vitro, in cell cultures, and in vivo, in mice xenografts. Permeabilization was achieved by irradiation with near-IR $658 \mathrm{~nm}$ laser light, and it was found to be dependent on the percentage of photosensitizer, irradiation intensity and exposure. The results obtained in vitro and in vivo when using these liposomes loaded with Dox were very promising, which makes this DDS a candidate for anticancer therapy.In summary, light can be used as a stimulus to directly or indirectly trigger release from photosensitive liposomes. However, the modification of the liposomes is the major accomplishment in this field. For more detailed information on the different mechanisms by which photosensitive liposomes can be triggered and how the liposomal composition affects the release [146], the reader is encouraged to read more about photopolymerization [133, 146, 147], photosensitization [134, 137, 138, 148], photoisomerization [133, 146, 149], photooxidation $[133,136,146,147]$, or the degradation of photo cleavable lipids $[133,150]$.

\subsubsection{Magnetoliposomes}

The use of magnetic fields as a triggering technique, by which a controlled release can be induced, was already mentioned in previous sections. Magnetic materials were first 
used for hyperthermia, as initially proposed by Gilchrist et al. [79]. They proved to be the ideal hyperthermia inducing system since they are non-invasive, tissue-specific and capable of precise, localized, high-intensity heating of deep tissues (reviewed in [55]).

Drug delivery driven by magnetic fields was first proposed by Freeman and co-workers [151]. As discussed in section 3.1.1, the first attempts on the use of magnetic fields as a release trigger was as a heating mechanism that could be combined with TSLs into one DDS. A local high frequency AMF can be used at very high intensities to induce hyperthermia at the tumor side, thus causing drug release from the TSLs. However, at such intensities of magnetic fields, it is hard to control the amount of energy that is absorbed and converted into heat in the body, hence excessive exposure could lead to second or third order burns [152]. Furthermore, hyperthermia-induced by $\mathrm{AC}$ magnetic fields has several limitations, as reviewed in [153]. To avoid the harmful side effects, a group of scientist worked on the development of what is known as magnetoliposomes (MLs), which combinesliposomes as drug carriers and magnetic fields as a trigger. In this case, the magnetic fields are not used to induce hyperthermia that leads to drug release from TSLs, but they rather directly interact with the chemically modified liposomes specifically made to be sensitive to a certain parameter of the magnetic field.

Magnetoliposomes have been defined as liposomes that contain super paramagnetic nanoparticles in their composition. A super paramagnetic nanoparticle is one that interacts with magnetic fields and can absorb most of the field's energy, quickly converting it to heat $[154,155]$. Consequently, when such particles are added to liposomes and are placed in the radiation field of an AMF, their temperature increases, leading to a localized hyperthermia that affects the liposomes only. This DDS has several advantages due to its biocompatibility, chemical functionality and the fact that it can combine hyperthermia with drug delivery in the treatment of tumors [81]. The success of the MLs opened the field for researching magnetic nanomaterials that can be used along with magnetic field to cause hyperthermia, as reviewed in detail by Kumar et al. [48]. It is worth mentioning that the most widely used magnetic nanomaterialin MLs is the superparamagnetic iron oxide (Fe3O4), which produces the best release results when combined with AMF [156, 157]

In a recent review, Soenen and co-workers [158] clarified the ambiguity that is sometimes found in the literature concerning types of MLs. Hence, MLs may be considered as an individual magnetic nanoparticle (usually iron oxide with a diameter of about $15 \mathrm{~nm}$ ) surrounded by a phospholipid bilayer and, in this case, they cannot encapsulate any substance since their core is the magnetic nanoparticle itself. The same name is used to designate large unilamellar vesicles encapsulating several magnetic nanoparticles in their aqueous cores, which can also be used to encapsulate drugs. The last category is what will be reviewed here.

In the work of Babincova et al. [80], large unilamellar Dox MLs encapsulating stabilized colloidal gamma-ferric oxide, were tested for the possibility of inducing a controlled release by using an AMF as the trigger. The results showed that, by subjecting the MLs to this field with a frequency of $3.5 \mathrm{MHz}$ and an induction of $1.5 \mathrm{mT}$ for few minutes, the temperature of the solution was raised to around $42^{\circ} \mathrm{C}$, which was the transition temperature of the lipids used to synthesize the MLs. At this temperature, a noticeable drug release was observed which indicated the success of the experiment.

Tai and co-workers [82] used TSLs with carboxyfluorescein co-encapsulated with iron oxide nanoparticles and monitored the drug release in solution, in an in vitro gel phantom, and in vivo, in rat skeletal muscle. The application of an AMF triggered the drug release from TSLs, and the in vivo experiments confirmed that the method is minimally toxic and relatively safe. The work of Pradhan et al. [81] demonstrated a very interesting synergism between biological and magnetic targeting, by synthesizing folate receptor-targeted thermosensitive MLs for use in hyperthermia chemotherapy. The liposome formulation was DPPC:cholesterol:DSPEPEG2000:Folate (80:20:4.5:0.5 molar ratio) and a commercial aqueous solution of iron oxide magnetic nanoparticles was coencapsulated with Dox. Using a permanent magnetic field, these MLs were targeted to KB and HeLa tumor cell lines, and led to increased cytotoxicity when compared to Dox encapsulated in the regular stealth liposomes, folate-targeted regular liposomes and free Dox. The cytotoxicity was enhanced due to the magnetic-induced hyperthermia $\left(42.5^{\circ} \mathrm{C}\right.$ and $43.5^{\circ} \mathrm{C}$ ).

Amstad and co-workers [44] designed stealth MLs containing the super paramagnetic nanoparticle iron oxide incorporated in the membranes. These particles $(5 \mathrm{~nm}$ diameter) were individually stabilized with palmityl-nitro DOPA and mixed with DSPC and PEG to produce stable MLs with a Tm of around $54.6{ }^{\circ} \mathrm{C}$. Release was observed when a sample of these liposomes, encapsulating calcein, was subjected to a $230-\mathrm{kHz}$ AMF for $25 \mathrm{~min}$ period divided into 5 sessions. It was hypothesized that, when the iron oxide nanoparticles where subjected to the AMF, they absorbed the energy and converted it into heat that was dissipated directly into the lipid bilayer of the liposome causing the local temperature to increase to $\mathrm{Tm} \geq 54.6^{\circ} \mathrm{C}$. It was also observed that the liposomal structure was maintained during exposure to the AMF, suggesting that the calcein was released due to transient changes in the membrane permeability and not due to rupture or fusion of the liposomes. This allowed control of the space and time of the released dose, making this a possible DDS to be used in vivo.

So, we can conclude that, in the future, AMF might have the potential to be a good triggering means by which drug release can be controlled either through direct interaction with the liposomes or, as is the case with light, by using it to induce hyperthermia at the location where TSLs are present.

Thus far, the major triggering means used to induce drug release from liposomes, except for US, were discussed, focusing on defining the terms and describing the mechanism by which each trigger can be used. Numerous studies have been done for each triggering mechanism, and many types of 
liposomal compositions were developed in order to create successful DDS, since the liposomes have to be modified to be responsive to a specific trigger. This modification can be done through the addition of certain polymers, inclusion of superparamagnetic nanoparticles, introduction of certain lipids or peptides, adding amphiphilic molecules into the composition, or chemically modifying certain chemical bonds to make them sensitive to a trigger. As for the trigger, the objective is always to cause disruption to the liposomal membrane allowing the release of the encapsulated drugs. By combining the proper trigger with the proper carrier, side effects associated with chemotherapeutic agents can be avoided and their effectiveness in cancer treatment can be enhanced.

It is important to notice that, throughout the literature, several formulations and protocols have been described, to create liposomes that are sensitive to a certain trigger. It is also evident that liposomes with different chemical compositions can still be sensitive to the same trigger, yet differences between them show based on their release efficiency. For example, there are many types of copolymers that can be added to the liposomes to make them sensitive to $\mathrm{pH}$ level; the way they release is the same but the amount of the drug they can encapsulate and then release when triggered, may differ. The aim of this review was not to include all types of liposomal carriers but rather focus on the process of interaction between them and the specific triggering means. In the next section, a complete review on US as a trigger is introduced, with a discussion on US physics that will help understand the ways by which it can be used to control the drug release from liposomes [8].

\section{TRIGGERING RELEASE USING ULTRASOUND}

\subsection{The Physics of Ultrasound}

In recent years, US has been researched asbeing one of the best triggering techniques due to its safety and low cost. Nowadays US has many applications in the medical field, but perhaps the best known is its use as an imaging technique (including the imaging of embryos). This indicates how safe and recommended US is as a means of diagnostics and therapies [159-164]. In this section, a detailed investigation of US physics will be addressed, before elaborating on its application as a possible trigger mechanism in drug delivery.

Ultrasound consists of sound waves (pressure waves) with a frequency higher than $20 \mathrm{kHz}$. As a comparison, sound waves within the human hearing range have frequencies between $40 \mathrm{~Hz}$ to $20 \mathrm{kHz}$ [165-169]. The sound wave is a physical wave that needs a medium to travel through, unlike light or electromagnetic waves. Sound waves propagate by means of energy transfer between the molecules of the medium, which occur as the pressure changes from compression (high pressure) to refraction (low pressure). Ultrasound waves possess the properties of any wave, i.e., attenuation, reflection, refraction, amplification, absorption and scattering. Yet, US waves, also called acoustic waves, have the ability of propagating on the surface of matter without traveling through it [168-171].
Ultrasound waves consist of cycles of successive varying pressure values, similar to those shown in Fig. 6. There are two main types of pressure wave propagation, namely transverse and longitudinal, as described in Fig. 6. The upper image shows a longitudinal wave and the lower one a transverse wave. In longitudinal propagation, particles tend to move back and forth along with the direction of the wave. Therefore, if the wave is traveling from left to right on the xaxis, the particles themselves will be propagating parallel to the x-axis. On the other hand, in the case of the propagation of a transverse wave, also known as shear wave, the particles are stationary in the translational sense; however, they oscillate perpendicular to the propagation direction producing a wave behavior similar to that exhibited by sinusoidal waves $[166,170,172]$.

Usually the medium through which the propagation occurs is referred to as the fluid medium. Any fluid medium consists of a collection of particles that are always randomly floating and moving around the space. This motion is more restricted in the case of solids, it is moderate in liquids, and it is the least restricted in gases. The motion restriction is due to the density of the material: as the density increases, the number of molecules filling up a specific volume increases but the available space for each molecule to move within decreases. Consequently, the molecules, in more dense materials, are closer to each other and can easily collide. Fig. 7 shows the molecules as spheres distributed in the space. When no pressure is applied, there will be no pattern in the motion of the molecules, only random motion of the particles [173]. 
Once pressure is applied, the molecules are compressed

particles just oscillate in place to allow this propagation. The

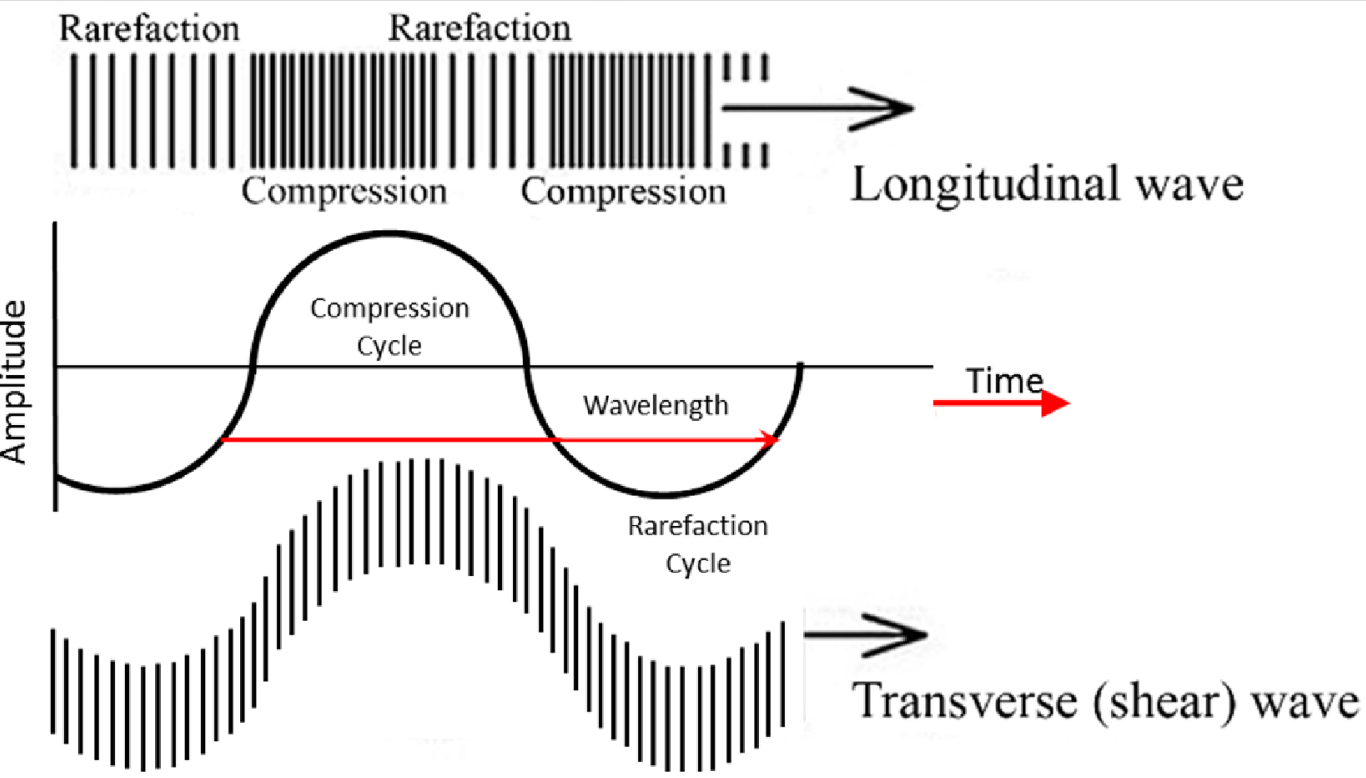

Fig. (6). Nature of acoustic wave propagation:shear and longitudinal.

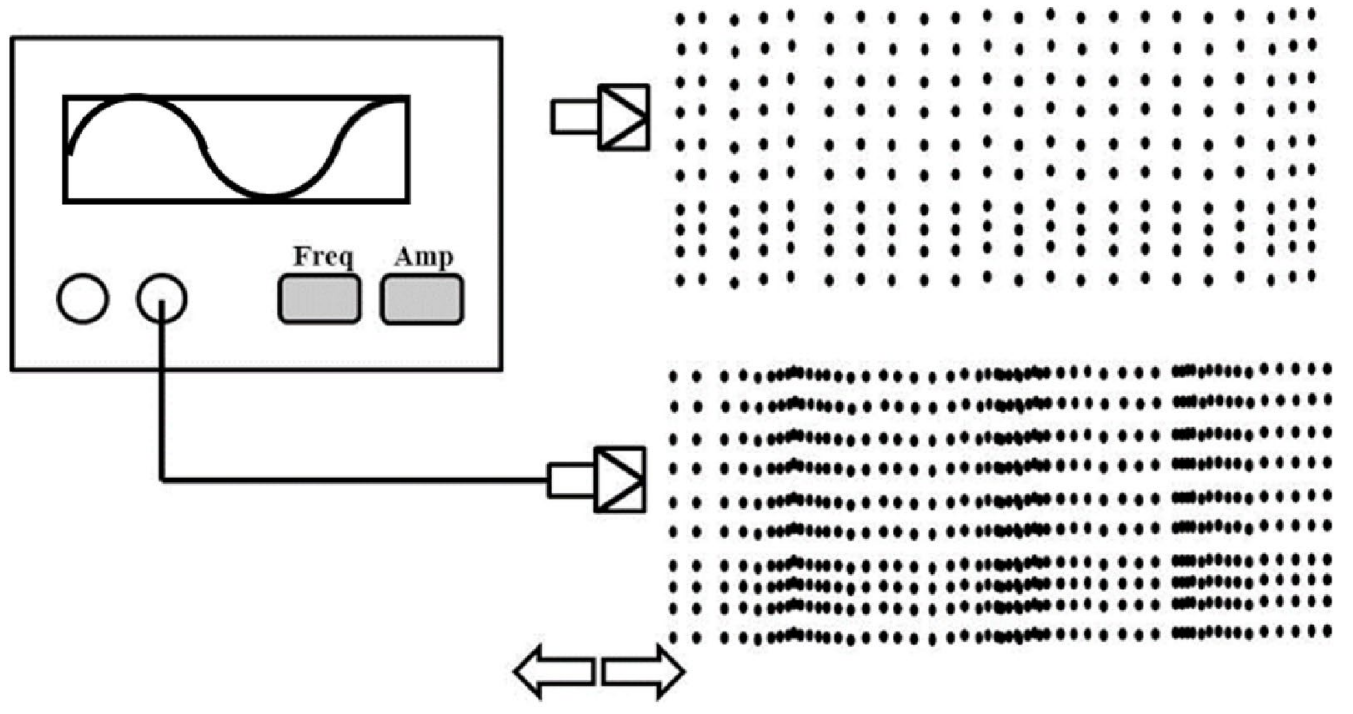

Fig. (7). Successive cycles of high and low pressure areas formed due to the motion of a piston in a medium of particles.

together forming a band-like shape corresponding to the intensity of the pressure wave. The band of molecules will then be accelerated in the direction of the wave propagation, but as molecules come in contact with the next line of molecules, energy transfer occurs. Eventually, the initial band of molecules that were in direct contact with the pressure wave will lose the gained energy and will start to divert from each other. This state is referred to as the rarefaction state [174176]. When a series of compression and rarefaction states are induced periodically into the fluid medium, a pressure wave can propagate smoothly as long as the fluid is clear of any obstacles. The region of compression corresponds to a region of high pressure, while the region of rarefaction corresponds to low pressure. It must be noted that only the wave propagates; particles in the compressed regions are moving forwards, and those in rarefaction are moving backwards.

Since solids have molecules that are tightly compacted, sound waves travel faster in solid media than in liquids and gases. In fact, air and vacuum are some of the worst sound conductors and the speed of sound in these media is considerably low compared to other media, for high frequency sound waves. In air, the molecules are distant from each other, hence the particles of the medium take a long time to come close to each other, thus the energy transfer rate is slower. Due to this slow motion of particles, the acoustic energy band that forms a compression region hardly forms, which in turn presents difficulties in the face of the acoustic wave propagation. For low frequencies, the scenario is different. As 
an example, the human voice propagates through the air, and can be clearly heard when people are talking. Hence, low frequency sound waves can travel through the air but they fade quickly due to losses $[177,178]$.

Acoustic waves are a form of pressure waves that propagate as described above. When the successive pressure variation is compared to a normal sinusoidal wave, the high pressure is equivalent to the upper peaks of the sinusoid, and the low pressure is equivalent to the lower peaks. Hence, speed, wavelength, frequency and amplitude of the pressure wave are parameters that can be used to characterize acoustic waves. Fig. 8 shows an acoustic wave represented as a sinusoid with a wavelength $(\lambda)$, a frequency $(1 / \mathrm{T})$ and a speed which is the product of frequency and wavelength. In most soft tissues, the velocity of US is about $1540 \mathrm{~m} / \mathrm{sec}[166,167,174,179,180]$.

Ultrasound waves are easily generated using a system that consists of an actuator that produces an alternating current at adjustable frequencies based on the application, and a wire that carries this AC current to a probe used as the terminal (from which the pressure waves are emitted [181]). Knowing the physics of US have helped widen the spectrum of applications. Frequency is one of the main controllable parameters that determineswhich application the US can be used for [181, 182]. Table 1 shows the ranges of ultrasonic frequencies and their corresponding applications.

To understand more about the role of frequency in US applications, imaging can be taken as an illustrative example. Frequencies of $1 \mathrm{MHz}$ and greater are required to obtain US suitable for diagnostic imaging [173, 179, 183]. This is because, in imaging the human body, the US wave has to penetrate to a certain depth and reflect back while still having some of its initial intensity. By adjusting the frequency, the proper depth can be reached based on the fact that the higher the frequency, the lower the penetration depth (described earlier in equation (2)). Also, at higher frequencies, the wavelengthsare shorter, which allows for more precise and detailed imaging. However, there is always a tradeoff between depth and details, because of the direct relation between the frequency and the wavelength based on equation (3). Since the speed of US in the human tissue is assumed to be constant, changing either of the two will lead to an opposite change in the other $[160,165,173,179,183]$.

$v=f * \lambda$

By examining Table 1 carefully, it can be seen that applications have overlapping ultrasonic frequency ranges. Tissue ablation and organ imaging have an overlap in the range of 1-1.5 MHz. Yet, for imaging, the intensity of the US is very low, barely enough to allow the US to propagate through and reflect back with enough intensity that can be used to form the image. On the other hand, for tissue ablation, higher intensities are used that lead to hyperthermia at the targeted area. Lower intensities cannot achieve the required increase in temperature. Thus, although the frequency ranges overlap, the application is determined by the intensity [173, 179, 182-189].

Another controllable parameter is the mode of operation $[186,188,190]$. There are two main modes of operation: continuous mode or pulsed mode [191]. In continuous mode, the US wave is generated and applied continuously for a certain period of time. In the pulsed mode, the wave is generated in a cycle of on and off periods. The mode also plays a role in determining the application even if there is an overlap in the frequencies. For example, although there is an overlap in the frequency ranges between kidney stone shattering and tissue ablation and both of them use US within the same intensity range (Table 1), kidney stone shattering uses a pulsed mode to cause the breakdown of the stones while tissue ablation requires the application of US continuously for long periods, allowing for the tissue to overheat. Hence, these three controllable parameters are the key to understanding the ways by which US can be used in drug delivery and triggering release [191-195].

Another important aspect that must be analyzed and that helps in understanding the advantages and disadvantages of US in DDS is the reflection phenomenon, which occurs when a sound wave is transferred from one material to another. Each material has a parameter called the acoustic impedance, which is specific for it. Frequently there is a mismatch between the impedances of any two contacting materials, which occurs at the boundary, and causes some of the wave to pass through, while the rest is reflected. The amount of reflection is determined by the reflection coefficient, which is, by definition, lower than one. This value indicates the percentage of the wave that will be reflected in terms of intensity. Let the impedance of the first material be $Z_{l}$ and that of the second be $Z_{2}$. In the case of normal incidence, the amplitude reflection coefficient, $R$, can be derived as follows $[170,171,173,180]$ :

$$
R=\frac{Z 2-Z 1}{Z 2+Z 1}
$$

Using the amplitude reflection coefficient, the amount of energy, depicted in the intensity parameter, that will get through from one medium to the other can be approximated as a percentage, by finding the normal incidence intensity transmission coefficient calculated as follows: 


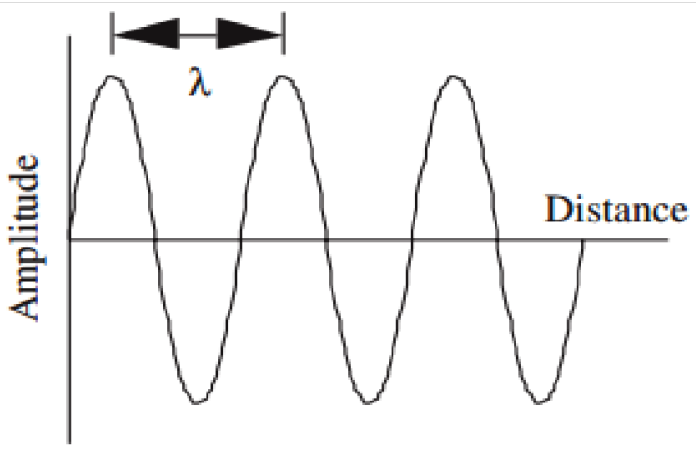

(a)

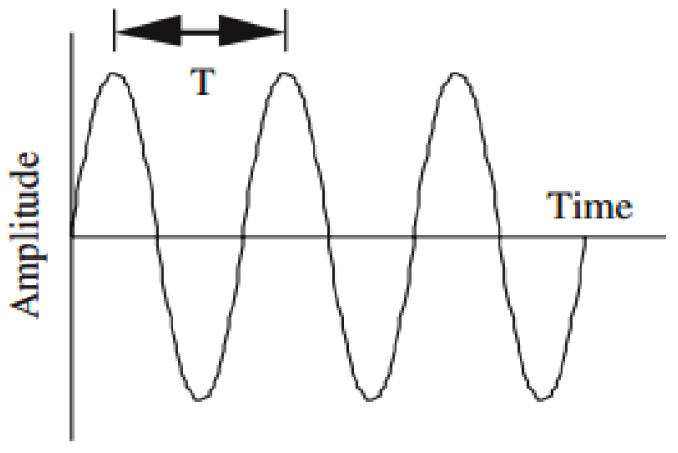

(b)

Fig. (8). Representation of a sinusoidal wave. The wave is characterized by a certain wavelength $\lambda$ and a frequency $1 / T$.

Table 1. Ultrasound: range of frequencies and respective applications [24].

\begin{tabular}{|c|c|c|c|}
\hline Frequency & Applications & Device & Description \\
\hline $30-150 \mathrm{kHz}$ & Dentistry & Micro Probes & Debris removal \\
\hline $0.1-1 \mathrm{MHz}$ & Kidney stone shattering & Lithotripter & Shattering of kidney stones through the absorption of ultrasound \\
\hline $0.5-1.5 \mathrm{MHz}$ & Tissue ablation & $\begin{array}{l}\text { High Intensity Focused } \\
\text { Ultrasound (HIFU) }\end{array}$ & $\begin{array}{l}\text { Heating of tissues to the point where cells start to die; intensities are high } \\
\text { enough to cause the temperature to rise above } 42^{\circ} \mathrm{C}\end{array}$ \\
\hline $0.7-3 \mathrm{MHz}$ & Physiotherapy & Normal Probes & $\begin{array}{l}\text { Heating certain locations of the body to help clearing internal organs from } \\
\text { unwanted cells, such as surface cancer cells; intensity used is moderate } \\
\text { enough to heat up the surface of the skin and few mm in depth. }\end{array}$ \\
\hline $1-20 \mathrm{MHz}$ & Organ imaging & Ultrasonic imaging & $\begin{array}{l}\text { Lower frequencies in this region are used to capture images of deep organs } \\
\text { such as liver and kidneys. Higher frequencies are used for imaging } \\
\text { superficial areas such as muscles and brain. Intensities used are low just } \\
\text { enough for a clear wave to be reflected back to the source. }\end{array}$ \\
\hline
\end{tabular}




$$
\text { It }=\left.\left.|1-| R\right|^{2}\right|^{*} 100 \%
$$

Beside the reflections that happen due to the mismatch in the impedance, impedance itself causes losses in the intensity of the passing wave. This loss is referred to as attenuation due to absorption or energy change, and it is another factor that governs the possibility of using US as a triggering means. When a wave passes through a medium, some of the transmitted energy changes into other forms, e.g. heat energy in the case of tissue ablation. This change will in turn lead to a

Table 2. Average attenuation coefficients in tissue [165].

\begin{tabular}{|c|c|c|c|}
\hline Frequency (MHz) & $\begin{array}{c}\text { Average Attenuation Coefficient for } \\
\text { Soft Tissue (dB/cm) }\end{array}$ & $\begin{array}{c}\text { Intensity Reduction in } \\
\mathbf{1} \text { cm Path (\%) }\end{array}$ & $\begin{array}{c}\text { Intensity Reduction in } \\
\mathbf{1 0} \mathbf{c m} \text { Path (\%) }\end{array}$ \\
\hline \hline 2.0 & 1.0 & 21 & 90 \\
\hline 3.5 & 1.8 & 44 & 98 \\
\hline 5.0 & 2.5 & 58 & 99.7 \\
\hline 7.5 & 3.8 & 68 & 99.98 \\
\hline 10.0 & 5.0 & 99.999 \\
\hline
\end{tabular}

loss in the energy hence the amplitude of the pressure wave will decrease as the distance traveled through the medium increases. This can be modeled using equation (6). As can be observed, the attenuation is exponential and it differs from one medium to the other; hence, the attenuation factor $\beta$ is specific to each medium [173],

$$
I 2=I 1 * \exp (-2 \beta \Delta x)
$$

where, $I_{2}$ is the intensity at $x_{2}$, and $I_{1}$ is the intensity at $x_{1}$, given that $\Delta x=x 2-x 1$ where $x$ is the position of the wave.

Furthermore, the absorption is linearly proportional to the frequency, so at higher frequencies, absorption is higher, hence the distance that the wave travels decreases. This distance is called the depth of the signal in a medium, and it is another factor that decides which frequency to be used for which application. Yet, the absorption coefficient varies from one medium to the other, being lower in solids and higher in gases. The absorption coefficient and the depth of the signal are the two main factors that limit the applications of US in air. As mentioned earlier, air is one of the worst media for acoustic waves' propagation in the case of high frequencies, hence if air is present in the medium of ultrasonic application, the system usually fails. Also, since air has very high acoustic impedance, there will always be a vast mismatch between it and any other medium, which causes the reflection of most of the wave.

As the frequency increases, the penetration depth decreases because the energy from the acoustic wave is more easily absorbed at higher frequencies. Also, depending on the type of medium, the amount of absorption can be determined. Table 2 shows the relation between the attenuation coefficient and frequency.

The attenuation coefficient and the acoustic impedance are important when dealing with the human body and analyzing how it interacts with US. The human body consists of stacked layers of tissue - skin, fat, muscle and bones, each one with its own acoustic impedance (Table 3). Hence, an acoustic wave passing through these layers will exhibit attenuations and reflections as it propagates, as discussed earlier. This is critical because the attenuation, which is usually due to absorption, will cause the tissues to heat up, as the absorbed energy is converted into thermal energy. This is the basis of several of the current applications of US. The accurate knowledge of the proper values is critical to avoid overheating and damaging the skin. Also, due to the mismatches, which are small, some of the wave is reflected. These reflections work as the basis for the imaging of human organs. The knowledge of the mismatches helps in calculating the proper frequency and intensity for the imaging process. It is worth noting that there is a small mismatch in the acoustic impedance of the skin, muscle and fat, but the mismatch in bones is enormous. This is because bones are considered solids, while the other tissues are considered liquid and they contain a high percent of water.

This basic information about how US interacts with human tissues is critical for the understanding of the next part of this review, which discusses the mechanisms by which US is used to cause drug release from liposomes. The

mechanisms are explained in detail with reference to the physics of US discussed in the above sections.

\subsection{Interaction of Ultrasound with Liposomes}

Ultrasound, a potential trigger for drug release from nanocarriers, is gaining significant attention in creating successful DDS. Nonetheless, similar to other triggering means, the drug carriers must be modified to become more responsive to acoustic waves. Liposomes modified to increase their acoustic sensitivity are referred to as echogenic or Acoustically Activated Liposomes (AAL). The modifications depend on several US parameters, including intensity and frequency of the triggering acoustic wave, and are aimed to obtain the most efficient release. For example, high intensity US at a low frequency causes an increase in the temperature of the medium, hence, liposomes triggered using this technique are synthesized/designed torespond to hyperthermia. This section introduces several types of echogenic liposomes and the mechanisms by which US induces drug release. By 
understanding the different ways US interacts with a liposome and the advantages and

Table 3. Attenuation of human tissue at $1 \mathrm{MHz}$. Adapted from [174, 196].

\begin{tabular}{|c|c|}
\hline Human Tissue & Attenuation (dB/cm) \\
\hline \hline Blood & 0.18 \\
\hline Fat & 0.6 \\
\hline Kidney & 1.0 \\
\hline Muscle (across fibers) & 3.3 \\
\hline Muscle (along fibers) & 1.2 \\
\hline Brain & 0.85 \\
\hline Liver & 0.9 \\
\hline Lung & 40.0 \\
\hline Skull & 20.0 \\
\hline Lens & 2.0 \\
\hline Aqueous humor & 0.022 \\
\hline Vitreous humor & 0.13 \\
\hline
\end{tabular}

disadvantages of each, new, and possibly better, liposomal chemical formulations can be developed, leading to better DDS.

\subsubsection{Hyperthermia}

Liposomes are made up of a lipid bilayer which, similarly to cell membranes, gives them an acoustic impedance, close to that of muscle tissues. This impedance means that, as the acoustic wave passes through the liposomes, there will be some energy dissipation absorbed by the liposome. This energy is converted into heat, which raises the temperature of the lipids. If the liposome is designed with a specific transition temperature (e.g. the TSL discussed earlier) hyperthermia can be used to achieve this temperature, causing the lipid bilayer to transition from the LO state to the SO state, introducing pores within the shell, which in turn allows for the release of therapeutic agents. Hyperthermia, as stated in the work of Schroder et al. [24], can be achieved using HIFU, at frequencies higher than $0.5 \mathrm{MHz}$, with intensities that can reach up to several hundreds $\mathrm{W} / \mathrm{cm}^{2}$.

The intensity of an acoustic wave is defined in terms of power over unit area. The intensity is affected by the amplitude of the wave (i.e., the larger the amplitude of the generated wave, the higher the intensity) and also by the size of the area that it is directed at. Hence, for hyperthermia applications, HIFU is used, since the ultrasonic wave is focused onto a small area at large amplitudes, thus very high-power-to-area ratios can be achieved. Nonetheless, not all ultrasonic waves can be focused. There are two factors, namely diffraction correction factor and the directivity of the beam, which govern the process of focusing a beam [165167, 170, 171, 179]. Since both factors are dependent on the physical parameters of the transducer, which in turn are dependent on the resonance frequency, it was observed that higher frequencies are easier to be focused than lower ones.

When using HIFU with TSL, and depending on the size of the liposomes, power and frequency can be optimized to yield the required intensity at the desired location and the needed depth within the body. The lower the frequency, the lower the intensity needed to achieve the targeted hyperthermia, since low frequency US (LFUS) can penetrate further hence more energy can be absorbed by the liposomes and the human cellular structure at the tumor site. On the other hand, at higher frequencies, higher intensities are needed to reach the tumor site and achieve the required hyperthermia $[50,51,58,70,197$, 198]. Hence, similar energy absorptions can be achieved when using low intensity at lower frequencies, and high intensity at higher frequencies; however, there is always the tradeoff of causing damage to the healthy tissue being irradiated. For example, if the decision was to use high frequency at high intensity, the outer layers of the body will be subjected to extremely elevated temperatures as the US will lose energy faster and at shallower depths. On the other hand, lower intensities can penetrate further avoiding over heating of the outer layers of the body, but this might be at the expense of more dispersed (unfocused) beam of US. Therefore, there is a need to optimize frequency and power density when US is used to induce hyperthermia. Such optimization has been adequately addressed in literature [199-202]. Hence, although hyperthermia can be easily introduced using lower frequencies at lower intensities, this frequency range is hardly focused, which makes it difficult to attain the required intensity at the target point. Further, medical US is within the range of 1 to 15 $\mathrm{MHz}$, since US of lower frequencies interact more efficiently with the body tissues, and can cause severe hyperthermia that damages healthy tissues $[179,203]$

Another factor when optimizing the use of HIFU is the skin depth, which determines the penetration depth of the acoustic wave into the body. This is critical in the case of deeply localized tumors, since losses due to penetration have to be taken into consideration if a certain intensity is required at a deeper level in the human body $[58,204]$. For example, if an intensity of $10 \mathrm{~W} / \mathrm{cm}^{2}$ is required at a depth of $5 \mathrm{~cm}$ into the human body, the applied acoustic HIFU wave should have higher intensity to account for the losses as the wave propagates into the body. As discussed earlier, the losses may arise due to reflections caused by the impedance mismatches between the different layers in the body, and also due to the absorption of some of the energy by the cells. Both factors are dependent on the frequency of the wave: the higher the frequency, the higher the losses and the lesser the penetration ability. The proper frequency should be chosen carefully so that the required intensity can be reached at the desired site without greatly increasing the intensity at the surface, since 
this could lead to tissue damage of the skin and other structures.

Besides being used as a trigger for drug release from TSL, hyperthermia induced by HIFU can also be used as a direct means to induce the death of cancer cells. Cancer cells subjected to temperatures above $42^{\circ} \mathrm{C}$ die, hence, if the cancer is superficial or directly on the skin, it can be treated by HIFU without the need for chemotherapy [205-207].

\subsubsection{Cavitation}

Another form of ultrasonic triggered release depends on cavitation. Cavitation is a natural phenomenon that occurs when a wave is incident on a bubble filled with a liquid that has the same resonance frequency as that of the incident wave [208]. In this case, the bubble will start to oscillate at its resonant frequency. This is a well-known phenomenon since everything has a resonant frequency of its own. For example, in 1940, Tacoma Narrows Bridge, in Washington, USA, collapsed when the passing winds caused it to resonate at its resonance frequency [209]. At this frequency, the particles tend to vibrate in a harmonious way. For example, if the vibrations were to the right, all the particles of the bridge would be moving to the right. If the vibrations were to the left, all the particles would be moving to the left. When the amplitude of the vibration was high enough, all the particles were vigorously swinging to one side or the other, and the vibration could no longer be sustained by the bridge, which caused its collapse. The same concept applies to the cavitation of a microbubble. If an acoustic wave is applied at the resonance frequency of the bubble, it will start to oscillate along with the wave. When the wave is in the low pressure stage, the bubble will be stretched, and, as the wave cycle goes from low pressure to high pressure, the bubble will start to compress gradually until it reaches the peak of compression, which corresponds to the maximum peak of the pressure wave. Microbubbles have certain tolerance points after which they can no longer get compressed or stretched based on the encapsulated gas. When the applied wave reaches peak pressures higher than the tolerance point, the microbubbles will not be able to oscillate and will burst, generating an intense shock wave that can shear open nearby cells. Also, this collapse is accompanied by the generation of very high temperatures, which can reach up to thousands of Kelvins [210]. While the bubble is oscillating with the wave, the cavitation is referred to as stable cavitation, while in the case of a bursting bubble, it is referred to as inertial or transient cavitation $[211,212]$.

In drug delivery, the transient cavitation phenomenon is of interest, since if the liposomes are close to microbubbles that undergo transient cavitation, the shock wave produced may cause the liposomal membrane to open thus allowing for the drug to diffuse out of the nanocarrier (Fig. 9). Also, if the bubble is close to the tumor site, the shock wave as well as microjets of liquid can lead to the disruption of the cell membranes, allowing the released drugs to enter and accumulate into the cells. This greatly enhances the performance of a DDS. These shooting jets occur when one side of a bubble is close to a cell or tissue. In this case, the burst bubble causes liquid jets rather than shock waves [213]. This type of collapse, called asymmetrical collapse, only occurs when the motion of the bubble is restricted from only one side, while the other side is free to oscillate. The collapse happens on the free side and propagates towards the inner side giving rise to a directed shock wave, which, unlike the normal shock wave which propagates spherically with the center being the collapsed bubble, propagates along a straight line. Thus, all the energy is directed towards one point, in other words focused on the desired location [214]. If these liquid jets are directed towards a cell or tissue, they can cause extensive damage. Therefore, a combination of both types of bubble collapse leads to an enhanced DDS [215].

In summary, the cavitation-induced release from the liposomes is triggered due to either the shear wave produced from the collapse, directed or not, and/or due to the elevated temperatures that are generated in the process.

As discussed earlier, carriers designed to be triggered by this method are usually either dependent on naturally occurring bubbles in the vicinity of the targeted site, or make use of manually introduced microbubbles in the vicinity of the tumor, or must be loaded with nano-bubbles that can oscillate and cause cavitation. The problem with the first method is the fact that the sizes of naturally occurring bubbles are hardly known except through sophisticated means, e.g. imaging. This presents a constraint on the choice of the frequency as the resonance frequency of a bubble is dependent on its size. If the size is unknown, the only way to cause cavitation is through trial and error, hence different frequencies and power intensities have to be used until transient cavitation is achieved. Higher intensity means higher risk of causing damaging hyperthermia and this is undesirable. This tradeoff between complexity and side effect called for a controlled size of microbubbles used to induce cavitation. Thus, researchers created microbubbles of a fixed size and introduced them into the tumor site [216]. Since the size of these microbubbles is known, their resonance frequency can be calculated, hence, transient cavitation and drug release can be controlled. In the case of AALs, these are best triggered using low frequency and intensity US. At low frequencies, in the range of 20 to $500 \mathrm{kHz}$, small intensities in the range $0.5-10 \mathrm{~W} / \mathrm{cm}^{2}$ can be used; the higher the frequency used the higher the intensity needed, which leads to the use of HIFU [217]. 

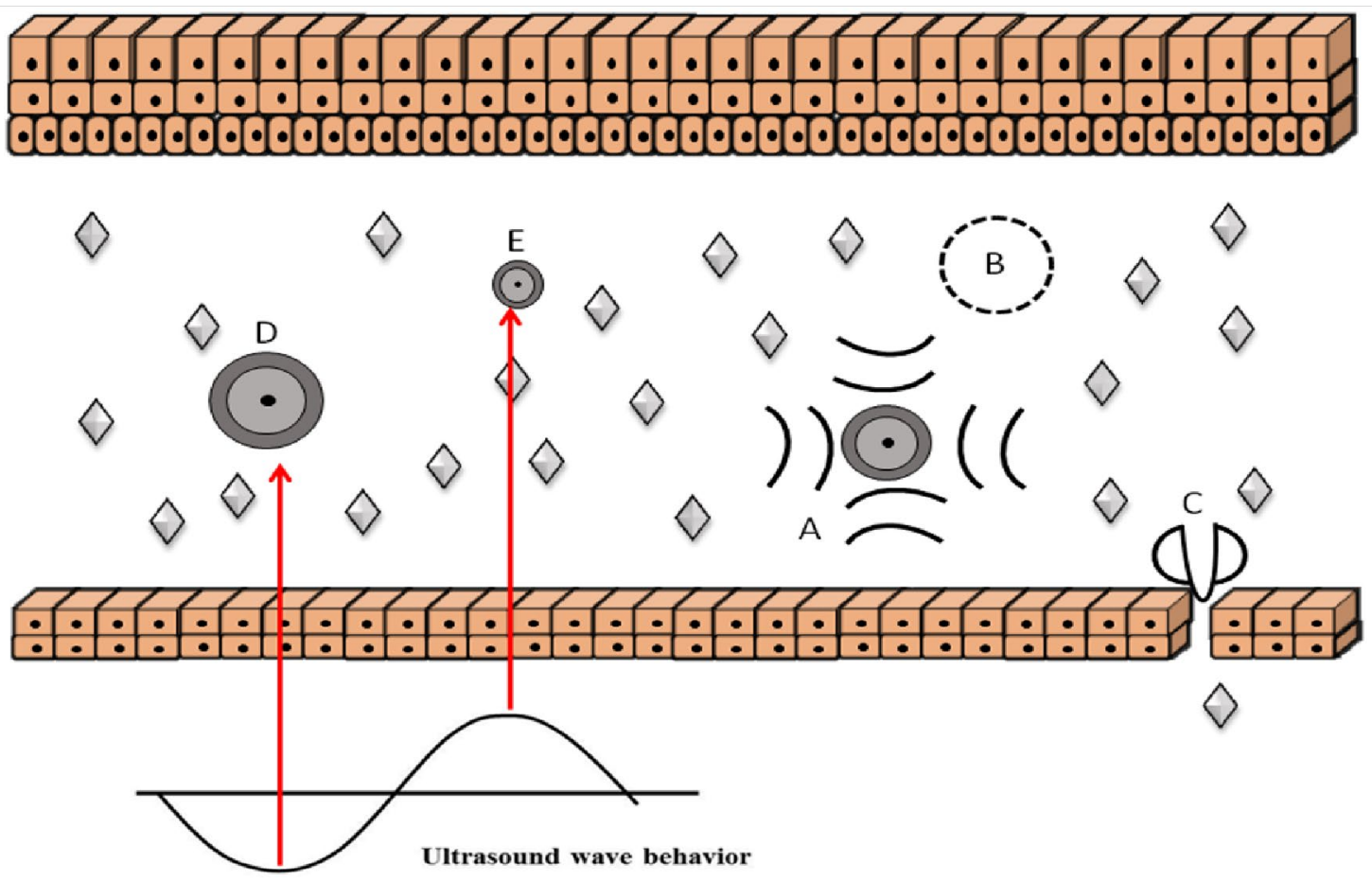

Fig. (9). Different types of cavitation processes. Microbubble (A) is undergoing stable cavitation, while (B) is undergoing collapse cavitation, producing a shock wave that can shear open the epithelial cells, and may lead to drug uptake if loaded liposomes are in the region. Microbubble (C) suffered an asymmetric collapse, generating a directed shock wave which sends a microjet in the direction of the cell bed, puncturing it. Bubbles (D) and (E) are shown alongside a graph depicting an ultrasonic wave, to illustrate the effect of the compression and

rarefaction phases on the bubbles' behavior.

There are two methods by which bubbles can be manually introduced. They can be either encapsulated inside the liposomes, along with the drugs, or they may be placed in the membrane, sandwiched between the lipid bilayer, as described by Huang and MacDonald [40, 218]. Fig. 10 clearly shows the location of the bubble in the membrane as well as the place of

the loaded drug. Using such AAL, transient cavitation can be induced and the drug can be released easily into the surroundings. These carriers can be further enhanced to make them actively targeted, so they can be directed to cancer cells and the release can be triggered directly into the cytosol.

However, the smaller the bubble the higher its resonance frequency and the higher the intensity it needs to undergo transient cavitation. This is because, as the frequency increases, larger bubbles tend to be less sensitive to the acoustic wave and may not even oscillate. The only bubbles that would oscillate are those with very small diameters. A governing factor that measures the possibility that transient cavitation would occur is called the mechanical index, which is calculated as follows,

$M I=\frac{P_{n e g}}{f^{0.5}}$

where $P$ neg is the peak negative pressure of the acoustic wave (in $\mathrm{MPa}$ ) and $\mathrm{f}$ the frequency (in $\mathrm{MHz}$ ). As shown in this equation, the mechanical index is a function of both the frequency and the pressure amplitude of the incident acoustic wave, so as the frequency increases, the MI decreases which mean that the possibility of cavitation decreases. To counteract the effect of increasing the frequency, the intensity should increase extensively. For cavitation to be probable, the MI should be 0.7 or higher $[219,220]$. At lower frequencies this can be easily achieved by using very low intensities. Optical and acoustic methods were used to track the destruction that takes place when an acoustic wave is directed towards phospholipid-shelled microbubbles. Experiments showed that the bubbles are destroyed by either one of two main mechanisms: acoustic dissolution at low acoustic pressure, or fragmentation of the parent bubble into two or more daughter bubbles at high pressure. Once the US is applied, the gas inside the microbubbles starts to oscillate causing the whole bubble to oscillate. If the oscillation is stable, currents are formed in the aqueous environment around the bubble. The work done by Mehier-Humbert and co-workers [221] showed that at a frequency of $2.25 \mathrm{MHz}$, small bubbles start to oscillate. As the intensity increases, the bubbles undergo transient cavitation which causes some damage to the surroundings. Yet, these experiments were done in vitro and the pressure used at this frequency was $300 \mathrm{kPa}$. The MI for these parameters is around 0.2 which is less than the 0.7 threshold. Further research was done at a frequency of $1 \mathrm{MHz}$ and a pressure of $1.3 \mathrm{MPa}$, yielding a MI of 1.3. Although transient cavitation is possible at this MI, damage in this case was lower and low bubble collapse could be observed, with some bubbles expanding from $2 \mu \mathrm{m}$ to approximately $20 \mu \mathrm{m}$, when simulation experiments set the limit at a maximum expansion of $55 \mu \mathrm{m}$. These puzzling results were further analyzed by Oerlemans et 
al. [222] who suggested a different mechanism for the observed damage when high frequency US(HFUS) was used, as discussed in the next Section applied in the absence of external microbubbles. This suggested that, although the MI was high enough to cause transient cavitation, this was not the main mechanism behind

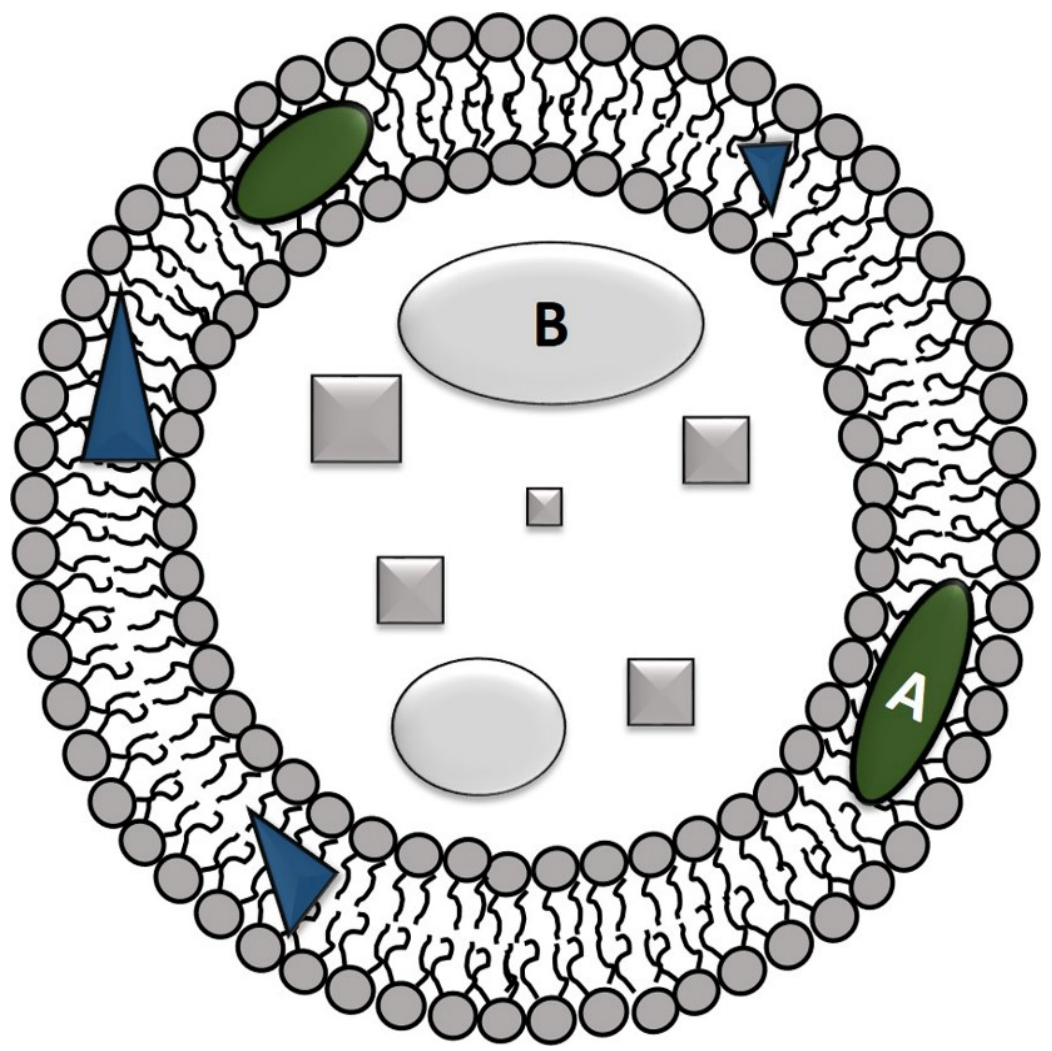

Fig. (10). Liposomes loaded with microbubbles. (A) Microbubble entrapped between the lipid bilayer of the liposome; (B) Air bubble encapsulated in the internal aqueous compartment of the liposome.

\subsubsection{Collisional Mechanism}

In 2013, a paper published by Oerlemans and co-workers [222] suggested a different mechanism for HIFU-induced drug release from nanocarriers. Experiments were conducted using TSL and non-TSL (NTSL) carriers, to exclude hyperthermia as the main mechanism of release. The sizes of the liposomes were between 97 to $139 \mathrm{~nm}$, and the chemical composition of the TSL made its $\mathrm{T}_{\mathrm{m}}$ around $42{ }^{\circ} \mathrm{C}$. Each type of liposome was loaded with a lipophilic compound (Nile red) and with a hydrophilic compound (Fluorescein), and both carriers were then subjected to a continuous wave of HIFU. When TSL were subjected to the HIFU waves for $15 \mathrm{~min}$, the temperature was raised above the $\mathrm{T}_{\mathrm{m}}$ and $80 \%$ release of the content was observed. However, in the case of the NTSL there was no release under the same conditions. Further, both carriers were then subjected to PW- HIFU (Pulsed Wave- High Intensity US) with an intensity of $20 \mathrm{~W}$ for $16 \mathrm{~min}$. This failed to increase the temperature above the $\mathrm{T}_{\mathrm{m}}$, yet a release of about $85 \%$ was observed for the TSL while a slight release of about $27 \%$ was observed for the NTSL. These results suggested that when HIFU is used, hyperthermia is not the main mechanism for release. The second part of the experiment was designed to eliminate cavitation as the main release mechanism. Designed microbubbles were introduced into the vicinity of the liposomes before PW-HIFU was applied, and the release levels observed were similar to controls where PW-HIFU was he observed release. The researchers proposed that the shear force exerted by the acoustic wave forces the liposomes to move at very high speeds and collide with each other and/or with the walls of the testing chamber. The results were similar for the release of the hydrophobic and hydrophilic compounds.

\subsubsection{Acoustic Droplet Vaporization}

The last mechanism by which US can interact with carriers to cause release is referred to as the acoustic droplet vaporization (ADV) and it is based on the expansion of nanoemulsions as the surrounding pressure changes. This mechanism applies to the previously described eLiposomes. This triggering method makes use of the restricted volume of liposomes, i.e., once they are formed, expansion is allowed but cannot exceed a certain limit without destabilizing the liposomal membrane and eventually causes their rupture. The $\mathrm{PCF}_{6}$ and $\mathrm{PCF}_{5}$ nanoemulsions have high internal pressure, hence when the surrounding pressure falls below their internal pressure the emulsions are allowed to expand which in turn increases the volume of the liposomes forcing some tension on the shell. This tension may lead to the formation of pores that will allow the drug to be released. Sometimes, the liposomes can be completely ruptured if the volume of the emulsion increases beyond the threshold point that the liposome can tolerate. The tolerance of a liposome is determined by its chemical composition, with expansion tolerances ranging from $2 \%$ to $4 \%$ [37, 38, 223, 224]. Ultrasound can be used as a trigger to change pressure, since it is a pressure wave made up of a periodic series of low and high pressures. 
Lin and co-workers[39] used HIFU to trigger the release from eLiposomes containing $\mathrm{PCF}_{5}$ nanoemulsionsand loaded with Dox, a carrier named eLipoDox. The exposure to HFUS ( 1 and $3 \mathrm{MHz}$ ) at low intensities of 1 to $5 \mathrm{~W} / \mathrm{cm}^{2}$ caused release, which was, in all cases, lower than that observed for $20-\mathrm{kHz}$ LFUS. The release with HIFU was relatively low, about $10 \%$ after $2 \mathrm{sec}$ insonation, increasing to only $15 \%$ after $30 \mathrm{sec}$. For these shorter times, no temperature increase was observed, for both frequencies and the power densities used, which indicated a mechanical action on the liposomes. For higher insonation times, up to $5 \mathrm{~min}$, the temperature rise for both frequencies at a power density of $5 \mathrm{~W} / \mathrm{cm}^{2}$, is significant, and release is increased $(50 \%$ for $1 \mathrm{MHz}$ and $60 \%$ for $3 \mathrm{MHz})$ due to the $\mathrm{PFC}_{5}$ phase transitioning to vapor. eLiposomes are promising nanocarriers to can easily be triggered using US, yet the technology is still new, hence more research is needed to optimize their use in US drug Delivery.

\subsection{Relevant Research}

In this section we summarize several recent in vitro and in vivo studies performed in acoustically enhanced chemotherapeutic delivery from liposomes.

In 2003, a study by Lin and Thomas [225] used stealth PEGylated liposomes encapsulating calcein to study the effect of sonication at $20-\mathrm{kHz}$ US (LFUS), on the drug release. The reported results showed an increase in calcein release which was attributed to the echogenic nature of the PEGylated liposomes, whereby US enhances the permeability of the nanovehicles.

A year later, a method was described to prepare AALs, and their hydrophilic encapsulation capacity and their sensitivity to US were investigated [40]. Release was achieved using $1-\mathrm{MHz}$ US at $2 \mathrm{~W} / \mathrm{cm}^{2}$ for $10 \mathrm{~s}$, and the authors concluded that this is a promising DDS.

Yuh and co-workers [226] conducted an in vivo using pulsed-HIFU in a mice model inoculated with $\mathrm{SCC} 7$, a murine squamous cell carcinoma cell line. A group of mice were treated with liposome-encapsulated Dox alone, while another group was treated with same formulation in conjunction with pulsed HIFU. The results showed that the mean Dox concentration in the tumors treated with HIFU was $124 \%$ higher than when the mice did not receive US treatment, supporting the possible effectiveness of this DDS in cancer treatment.

In 2007, a study examined the effect of pulsed-HIFU on low-TSL and Dox release was monitored in vitro and in vivo [227]. In vitro results showed a triggered 50\% release of Dox from the low-TSL, but not from regular (non-temperature sensitive) liposomes. In vivo studies using a murine adenocarcinoma model, showed that the combination of the TSL with noninvasive and nondestructive pulsed-HIFU, resulted in rapid release of Dox and was correlated with a significant reduction in the tumor growth rate.

In the same year, Schroeder and co-workers [228] published the results of a study that involved LFUS and sterically stabilized liposomes (SSL) to control the release of different payloads of drugs from three different liposomal formulations, with a similar size. The three types of liposomes were exposed to a short period of LFUS after which around $80 \%$ of their contents were released. The release amount was a function of the US amplitude and exposure time, and it was attributed to the formation of transient pores on the surface of the liposomes through which the drug was allowed to diffuse.

In 2009, the same group reported the results of an in vivo study that inspected the possibility of controlling drug release of cisplatin from nano-SSL (nSSL) using LFUS in micebearing murine lymphoma tumors [229]. The results showed that the group treated with nSSL and LFUS had superior therapeutic results compared to the groups where free cisplatin with or without LFUS were used, or when the tumor was treated with liposomes containing cisplatin without the use of LFUS. Additionally, the same study reported that the therapeutic efficiency of cisplatin was increased when the LFUS was used to induce the localized release of the liposomal drug in C26 colon adenocarcinomas develop in the footpad of $\mathrm{BALB} / \mathrm{c}$ mice.

On the other hand, in 201,0 a study was published on the use of 1.1-MHz HIFU to trigger fluorescent materials (FITC) release from liposomes ranging in size from 150 to $200 \mathrm{~nm}$ in diameter [199]. The results showed that a release of $21.2 \%$ was achieved after $10 \mathrm{~s}$ and around $70 \%$ release was observed after 60 s exposition to continuous wave US. Transmission electron microscopy showed that the large liposomes $(>100 \mathrm{~nm})$ were ruptured, while smaller ones $(<100 \mathrm{~nm})$ showed pores in their membrane.

In 2011, the group of Pitt et al. [230] published the preliminary results achieved when combining the use of liposomes loaded with therapeutic drug and low frequency/ low intensity US on tumors. The in vivo study used BDIX rats inoculated with rat colonic carcinoma DHD/K12 cells. The tumors were treated with Dox-loaded liposomes subjected to $20-\mathrm{kHz}$ US for 15 minutes period. The treatment was continued for 4 weeks. The results showed a statistically significant drug efficacy when the animals were subjected to US waves compared to the control case which involved the treatment of the rats with the same liposomes but without insonation.

In 2013, another in vivo study examined the use of liposomes synthesized using DOPE in treating mice inoculated with human prostate tumor cells (22Rv1). The tumor site was sonicated with $1.1 \mathrm{MHz}$ US wave. Results showed an enhancement in the release and the cellular uptake from these novel DOPE carriers compared to liposomes synthesized using hydrogenated soy phosphatidylcholine (HSPC) [231].

A recent study by Ninomiya and co-workers [232], presented a DDS consisting of liposomes modified with poly(NIPMAM-co-NIPAM), a temperature sensitive polymer, exposed to $1-\mathrm{MHz}$ US, power density of $0.5 \mathrm{~W} / \mathrm{cm}^{2}$. After 120 sec, the release of encapsulated calcein was observed. A similar release was detected when the liposomes were incubated at $42{ }^{\circ} \mathrm{C}$ for $15 \mathrm{~min}$. The study also involved in vitro experiments with the liver carcinoma cell line HepG2, using the same liposomes loaded with Dox. The cells were sonicated with $1-\mathrm{MHz} \mathrm{US}, 0.5 \mathrm{~W} / \mathrm{cm}^{2}$ for $30 \mathrm{sec}$, and $6 \mathrm{~h}$ after the 
exposure the cell viability was $60 \%$, significantly lower than in any of the controls (Dox thermosensitive liposomes with no US, Dox-loaded regular liposomes).

Rizzitelli and co-workers [233] also used pulsed highfrequency $3-\mathrm{MHz}$ US, but non-focused, to develop a MRIguided protocol to observe the release of a paramagnetic agent from liposomes. The protocol was validated in vivo on mice inoculated with B16 melanoma cell line, and it was observed that after 2 min insonation the MRI signal was enhanced by $35 \%$, confirming the release of the encapsulated paramagnetic molecule.

Further research and in vivo experiments were conducted and almost all results were positive and showed the effectiveness of combining US with drug carriers. By doing so, the side effects of the therapeutic agents are extensively avoided while the efficiency of the treatment is enhanced.

It is worth mentioning that, from the review done in this work, it was noticed that LFUS has an advantage over HFUS in terms of rate of release from normal liposomes and TSLs especially for in vivo applications. This is explained by the physics of US. As the frequency increases, the absorption rate of the energy increases which presents a challenge if HFUS is to be used to cause release from deep sites in the body. Consequently, to penetrate deeper into the body at high frequencies, the intensity has to be increased to levels that might be damaging to the human body. The next section reviews the advantages and the disadvantages of the different types of US in DDS.

\subsection{Advantages and Shortcomings of US as a Trigger in DDS}

The main goal of the trigger in a DDS is to be able to reach the tumor site regardless of its location in the body while being as localized as possible. As described earlier, US used in triggering drug release can be of a low or high frequency nature, and both have advantages and disadvantages.

An important advantage of LFUS is its higher penetration ability compared to that of US at higher frequencies. This penetration ability is a derivative of the absorption constant of the medium at different frequencies and is usually referred to as the skin depth, which can be calculated using equation (1), which shows an inverse relation between the penetration depth and the frequency. Hence, as the frequency increases, the penetration depth decreases. Furthermore, most of the echogenic liposomes were shown to be more sensitive to LFUS as the waves have the ability to much more energy compared to higher frequency. Also, at lower frequencies, there is a higher chance of interaction between US and available microbubbles. This is because most of those bubbles have diameters in the range of micrometers which is very close to the wavelength of the US wave at lower ranges of frequencies. On the other hand, high frequencies of US are easily focused either using a lens-like structure or by simply designing a focused probe. This helps in the treatment of cancer using DDS as the insonation is required to be localized.
However, according to the literature, US can actually induce cell death as its intensity increases. For example, Wang and co-workers [234] studied the bioeffects of increasing intensities of 1.1-MHz US on the myelogenous leukemia cell line K562. It was observed that at intensities 1 and $2.1 \mathrm{~W} / \mathrm{cm}^{2}$, cell death increased to around $14 \%$ and $40.7 \%$, respectively. The aim of the study was to find the intensity at which US will induce cell death, and it could be concluded that US can indeed cause harm to the human health tissues. Furthermore, HIFU has been recently used as a radiation therapy against prostate cancer; nonetheless, it was shown to be associated with some side effects such as urine leakage and possible infections in the prostate area [235].

The literature reports several studies that utilized US to increase the local tissue temperature and kills cells via hyperthermia. However, the human body is not a smooth structure. It is composed of layers, solid structures and fluids, which complicates therapeutic procedures. Fluids are usually filled with gas bubbles through which US propagation, and hence penetration is restricted. The case is the same with solids tumors through which US propagation is reduced. This nonsmooth nature of human tissue presents a challenge when a certain depth is to be heated to a certain temperature. Reflections, attenuation and absorption occur, to heat the tissues at a certain depth, US intensity is sometimes increased to overcome any obstacles, and this may lead to over-heating the more superficial tissues. For example, Hayes and coworkers [236] observed that $3-\mathrm{MHz}$ US can heat $0.5 \mathrm{~cm}$ deeper in the tissue than previously reported, which supports the inaccuracies that might happen when dealing with US.

Until recently, US was thought to be harmless to the human tissues, but in the last few years, more incidents of unwanted side effects were reported. For example, in 2006, an article published in Midwifery Today [237], discussed the possibility that US imaging during pregnancy may actually affect the embryo development, due to hyperthermia. A study by Ang and co-workers reported in PNAS [238] showed that neural migration in mice embryos is affected by exposure to US waves.

These examples, definitely call for further research to make sure that the ultrasonic parameters (including frequency, power intensity and pulse length) used are harmless to healthy tissue.

\subsection{Liposomes and Ultrasound: Clinical Uses}

Many liposomal drugs have been approved for clinical use, and several others are undergoing clinical trials.

However, to the best of our knowledge, no clinical trials are being conducted to test the combined effects of liposomes and US in cancer chemotherapy, yet. A phase II clinical trial named MRI Guided High Intensity Focused Ultrasound (HIFU) and ThermoDox for Palliation of Painful Bone Metastases, has finished recruiting participants but it did not begin yet (http://clinicaltrials.gov/ct2/show/study/ NCT01640847). The aim of the study is to evaluate if the combination of HIFU with ThermoDox ${ }^{\square}$ (Dox encapsulated in lysolipid thermosensitive liposomes) can effectively and safely reduce the pain of 
patients with bone metastases. Another study conducted at the University of Oxford aims at studying the targeted delivery of ThermoDox ${ }^{\square}$ by mild hyperthermia induced by HIFU, in patients with liver metastases from lung, breast or colorectal primary tumors (https://clinicaltrials.gov/ct2/show/NCT02181075). This trial is currently recruiting participants.

Some examples of liposomal-based drugs used for cancer therapy that have been approved to be clinically used or are undergoing clinical trials will now be discussed.

The first liposomal formulation that was approved for clinical use in Europe in 1990 (and in 1997, in the USA) was AmBisome [218, 239, 240]. One of the best known liposomal formulations that was FDA-approved in 1995, for the treatment of Kaposi's sarcoma found in AIDS victims, is Doxil/Caelyx [218, 239, 240]. Doxil consists of PEGstabilized liposome-encapsulated Dox and is currently used for the treatment of patients with progressing ovarian cancer and patients with multiple myeloma $[239,241,242]$. Several other liposomal formulations were approved for clinical use, such as DaunoXome (liposome-encapsulated daunorubicin, used for the treatment of Kaposi's sarcoma, FDA-approved in 1996), Myocet (non-PEGylated liposome-encapsulated Dox, used for the treatment of breast cancer, approved in 2000 in Europe and Canada, and undergoing clinical trials in the USA), and DepoCyt (liposome-encapsulated cytosine arabinoside, used for the treatment of lymphoma complications, FDA-approved in 1999) [243]. More recently, the FDA also approved a drug called Marqibo (vincristine sulfate liposome injection) to treat patients with a rare type of lymphoblastic leukemia [241]. The first commercially available liposomal formulation of paclitaxel, called Lipusu $^{\circledR}$, has been approved in China for the treatment of ovarian, breast, gastric and head and neck cancers [244].

Several liposomal formulations are currently undergoing clinical trials in the USA, for example: Onco-TCS, liposomal cytarabine, for the treatment of non-Hodgkin lymphoma (phase I/II trial); SPI-77, stealth liposomal cisplatin, for the treatment of head and neck, and lung cancers (phase III trials); and Lipoplatin, liposomal cisplatin, for the treatment of pancreatic, head and neck, and breast cancers (phase III trials) [243]. Paclitaxel formulations undergoing clinical trials have recently been reviewed by Koudelka and Turanek [244] and Nehate et al. [245]. Babu and co-workers recently reviewed the state of ongoing or completed clinical trials using liposomal formulations [241]. Comprehensive information about clinical trials can be retrieved from the US National Institutes of Health website (http://clinicaltrials.gov/).

\section{CONCLUSION}

This work presented a review on the types of triggered drug release from liposomes, with a focus on the use of US. Triggered liposomes can be categorized into several categories: temperature-sensitive, enzyme-triggered, pHsensitive, light-triggered, magnetoliposomes and echogenic liposomes. Liposomes of each category are modified chemically and/or physically so that they become sensitive to the intended trigger(s). Chemical modifications are related to the composition and constituents used to synthesize liposomes, while physical modifications usually involve the addition of a sensitive agent such as microbubbles or a supermagnetic material. By modifying the liposomes, the release of the encapsulated drug can be controlled.

In the past few years, work on DDS composed of a carrier and a trigger was taken a step further by incorporating the triggering as well as targeting features into one multifunctional system. By doing so, carriers adopt one of the three targeting levels that enable them to selectively choose cancerous cells over healthy cells, and stay within the tumor location. Then, by triggering the release of the drug from the accumulated nanocarriers, many of the toxic side effects of the drug can be circumvented; the higher the level of targeting the lower the side effects.

Ultrasound is considered one of the most desirable triggering means in DDS as it has many parameters that can be adjusted for the triggering purpose, even allowing its use to trigger the release from morethan one type of modified liposomes. For example, US can be used to induce hyperthermia, thus it can be used to trigger release from TSLs. Also, because US is a pressure wave, it is possible to choose the right frequency and intensity, to use its mechanical to shear openmany types of liposomes, regardless of its modification, especially in in vitro experiments. This characteristic of US also allows its use in the induction of release from a newly developed type of carriers, called eLiposomes. Similarly, the fact that US can induce cavitation, makes it very useful intriggering release from any type of liposomes.

Hence, US proves to be a useful, yet a safe means, by which triggering can be achieved. However, much optimization needs to be done to choose the parameters adequate for triggering the release based on the type of liposomes used. The solution to this optimization problem can be established by analyzing the physic of US, which was also reviewed in this paper.

In conclusion, the objective of a DDS is to reduce the side effects associated with the chemotherapeutic agents that might lead to the death of healthy cells, besides the target cancerous ones. The ultimate DDS is the one that can deliver the drug to cancer cells only and prevent the circulation of the excess dose in the blood. Carriers including liposomes solve the problem of circulation as they are used to encapsulate the drug for long periods of time. Then, by modifying liposomes, targeting can be achieved by conjugating ligands to their surface. Once liposomes bind to the receptors overexpressed on the membrane of cancer cells, they willenter the cell cytosolvia endocytosis. Afterwards, using the proper trigger, drug release can be controlled and contained within diseased cancer cells only.

\section{CONFLICT OF INTEREST}

The author(s) confirm that this article content has no conflict of interest. 


\section{ACKNOWLEDGEMENTS}

The authors acknowledge the financial support of the Faculty Research Grant Type 1, from American University of Sharjah (to G.A. Husseini).

\section{LIST OF ABBREVIATIONS}

AAL

AMF

$\mathrm{DC} 8,9 \mathrm{PC}$

DDS

DMPC
$=$ acoustically activated liposomes

$=$ alternating magnetic fields

$=\quad 1,2$ - bis (tricosa-10,12-diynoyl)snglycero-3-phosphocholine

$=\quad$ drug delivery systems

$=$ 1,2-dimyristoyl-sn-glycero3 phosphocholine
DPPC

DOPE

DSPC

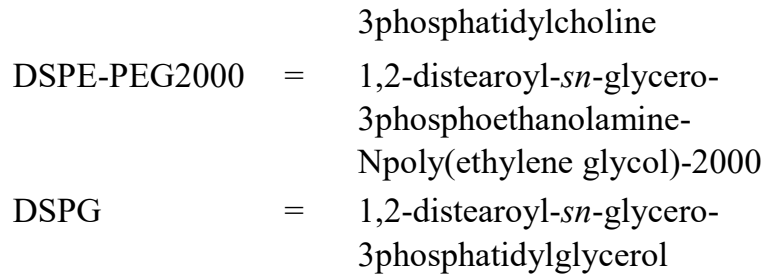

EPR

GUVs

HFUS

HIFU

LO

LFUS

LTSL

LUVs

MLs

MLVs

MPPC

PE

PEG

PFC5

PFC6

poly(Apr)

PLA2

poly(NIPAM)

poly(NIPAM-co- =

AA-co-ODA) poly(NIPAM-co- = MAA)

poly(NIPAM-co- =

ODA)

PTSL

$\mathrm{SnCI}_{2} \mathrm{Pc}(\mathrm{OBu}) 8=$

SO
$=$ 1,2-dipalmitoyl-sn-glycero3phosphocholine;

$=$ 1,2-dioleoyl-sn-glycero3 phosphoethanolamine

1,2-distearoyl-sn-glycero-

$=$ enhanced permeability and retention

$=$ grand or giant unilamellar vesicles

$=$ high frequency ultrasound

$=$ high intensity focused ultrasound

$=$ liquid-ordered (lipid) state

$=\quad$ low frequency ultrasound

$=$ lysolipid temperature sensitive liposomes

$=$ large unilamellar vesicles

$=$ magnetoliposomes

$=$ multilamellar vesicles;

$=$ 1-myristoyl-2-palmitoyl-sn-glycero3-phosphocholine

$=$ phosphatidylethanolamine

$=$ polyethylene glycol

$=$ perfluoropentane

$=$ perfluorohexane

$=$ poly(acryloylpyrrolidine)

$=$ phospholipase $\mathrm{A} 2$

$=\operatorname{poly}(\mathrm{N}$-isopropylacrylamide

copolymer of Nisopropylacrylamide, acrylic acid and octadecylamine NIPAM/methacrylic acid copolymer copolymer of isopropylacrylamide and octadecylamine

$=$ polymer-modified temperature sensitive liposomes

tin octabutoxyphthalocyanine dichloride

$=$ solid-ordered (lipid) state 


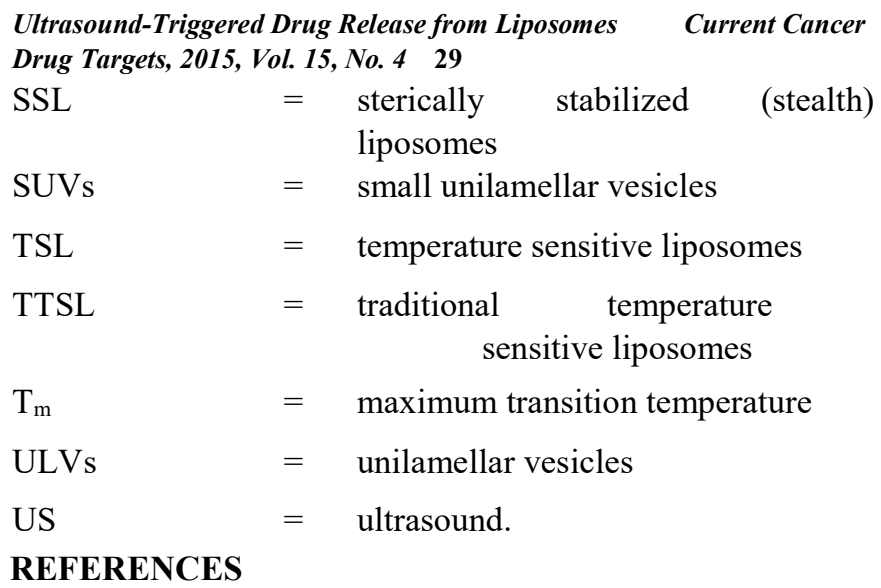

[1] Jemal, A.; Bray, F.; Center, M. M.; Ferlay, J.; Ward, E.; Forman, D. Global cancer statistics. CA. Cancer J. Clin., 2011, 61(2), 69-90.

[2] Parkin, D. M.; Bray, F.; Ferlay, J.; Pisani, P. Global cancer statistics, 2002. CA. Cancer J. Clin., 2005, 55(2), 74-108.

[3] Bradbury, R. H. In: Topics in Medicinal Chemistry: Cancer; Bradbury, R. H., Ed.; Springer-Verlag: Berlin. Heidelberg., 2007; Vol. 1, pp 1-17.

[4] DeVita, V. T., Jr.; Chu, E. A history of cancer chemotherapy. Cancer Res., 2008, 68(21), 8643-53.

[5] Lim, E. K.; Jang, E.; Lee, K.; Haam, S.; Huh, Y. M. Delivery of cancer therapeutics using nanotechnology. Pharmaceutics, 2013, 5(2), 294-317.

[6] Mozafari, M. R.; Pardakhty, A.; Azarmi, S.; Jazayeri, J. A.; Nokhodchi, A.; Omri, A. Role of nanocarrier systems in cancer nanotherapy. J. Liposome Res., 2009, 19(4), 310-21.

[7] Torchilin, V. P. Micellar nanocarriers: pharmaceutical perspectives. Pharm. Res., 2007, 24(1), 1-16.

[8] Bibi, S.; Lattmann, E.; Mohammed, A. R.; Perrie, Y. Trigger release liposome systems: local and remote controlled delivery? $J$. Microencapsul., 2012, 29(3), 262-76.

[9] Alexis, F. In: Handbook of Experimental Pharmacology: Drug Delivery; Schafer-Korting, M., Ed.; Springer-Verlag: Berlin. Heidelberg., 2010; pp 55-86.

[10] Benvegnu, T.; Lemiegre, L.; Cammas-Marion, S. New generation of liposomes called archaeosomes based on natural or synthetic archaeal lipids as innovative formulations for drug delivery. Recent Pat Drug Deliv Formul, 2009, 3(3), 206-20.

[11] Rawat, M.; Singh, D.; Saraf, S. Nanocarriers: promising vehicle for bioactive drugs. Biol. Pharm. Bull., 2006, 29(9), 1790-8.

[12] Bertrand, N.; Wu, J.; Xu, X.; Kamaly, N.; Farokhzad, O. C. Cancer nanotechnology: the impact of passive and active targeting in the era of modern cancer biology. Adv Drug Deliv Rev, 2014, 66, 2-25.

[13] Gujral, S.; Khatri, S. A review on basic concept of drug targeting and drug carrier system. Int. J. Adv. Pharm. Biol. Chem., 2013, 2, 130136.

[14] Matsumura, Y.; Maeda, H. A new concept for macromolecular therapeutics in cancer chemotherapy: mechanism of tumoritropic accumulation of proteins and the antitumor agent smancs. Cancer Res., 1986, 46(12 Pt 1), 6387-92.

[15] Maeda, H. The enhanced permeability and retention (EPR) effect in tumor vasculature: the key role of tumor-selective macromolecular drug targeting. Adv. Enzyme Regul., 2001, 41, 189-207.

[16] Maeda, H.; Wu, J.; Sawa, T.; Matsumura, Y.; Hori, K. Tumor vascular permeability and the EPR effect in macromolecular therapeutics: a review. J. Control. Release, 2000, 65(1-2), 271-84.

[17] Bergstrand, N. H. Liposomes for drug delivery: From physicochemical studies to applications. Uppsala University: Uppsala, 2003.

[18] Huynh, N. T.; Roger, E.; Lautram, N.; Benoit, J. P.; Passirani, C. The rise and rise of stealth nanocarriers for cancer therapy: passive versus active targeting. Nanomedicine (Lond), 2010, 5(9), 1415-33.

[19] Leucuta, S. E. Subcellular drug targeting, pharmacokinetics and bioavailability. J. Drug Target., 2014, 22(2), 95-115.

[20] Rajendran, L.; Knolker, H. J.; Simons, K. Subcellular targeting strategies for drug design and delivery. Nat. Rev. Drug Discov., 2010, 9(1), 29-42.

[21] Akbarzadeh, A.; Rezaei-Sadabady, R.; Davaran, S.; Joo, S. W.; Zarghami, N.; Hanifehpour, Y.; Samiei, M.; Kouhi, M.; NejatiKoshki, K. Liposome: classification, preparation, and applications. Nanoscale Res Lett, 2013, 8(1), 102.

[22] Deamer, D. W. From "banghasomes" to liposomes: a memoir of Alec Bangham, 1921-2010. FASEB J., 2010, 24(5), 1308-10.

[23] Lasic, D. Liposomes: synthetic lipid microspheres serve as multipurpose vehicles for the delivery of drugs, genetic material and cosmetics. Am. Sci., 1992, 80(1), 20-31.

[24] Schroeder, A.; Kost, J.; Barenholz, Y. Ultrasound, liposomes, and drug delivery: principles for using ultrasound to control the release of drugs from liposomes. Chem. Phys. Lipids, 2009, 162(12), 1-16.

[25] Garbuzenko, O.; Barenholz, Y.; Priev, A. Effect of grafted PEG on liposome size and on compressibility and packing of lipid bilayer. Chem. Phys. Lipids, 2005, 135(2), 117-29.

[26] Kumar, V. V. Complementary molecular shapes and additivity of the packing parameter of lipids. Proc. Natl. Acad. Sci. U. S. A., 1991, $88(2), 444-8$.

[27] Ulrich, A. S. Biophysical aspects of using liposomes as delivery vehicles. Biosci. Rep., 2002, 22(2), 129-50.

[28] Torchilin, V. P. Recent advances with liposomes as pharmaceutical carriers. Nat. Rev. Drug Discov., 2005, 4(2), 145-60.

[29] Immordino, M. L.; Dosio, F.; Cattel, L. Stealth liposomes: review of the basic science, rationale, and clinical applications, existing and potential. Int. J. Nanomedicine, 2006, 1(3), 297-315.

[30] Lasic, D. D. Novel applications of liposomes. Trends Biotechnol., 1998, 16(7), 307-21.

[31] Lasic, D. D.; Needham, D. The "Stealth" Liposome: A Prototypical Biomaterial. Chem. Rev., 1995, 95(8), 2601-2628.

[32] Woodle, M. C.; Lasic, D. D. Sterically stabilized liposomes. Biochim. Biophys. Acta, 1992, 1113(2), 171-99.

[33] Moore, N. W.; Kuhl, T. L. The role of flexible tethers in multiple ligand-receptor bond formation between curved surfaces. Biophys. J., 2006, 91(5), 1675-87.

[34] Li, X.; Ding, L.; Xu, Y.; Wang, Y.; Ping, Q. Targeted delivery of doxorubicin using stealth liposomes modified with transferrin. Int. J. Pharm., 2009, 373(1-2), 116-23.

[35] Javadi, M.; Pitt, W. G.; Belnap, D. M.; Tsosie, N. H.; Hartley, J. M. Encapsulating nanoemulsions inside eLiposomes for ultrasonic drug delivery. Langmuir, 2012, 28(41), 14720-9.

[36] Javadi, M.; Pitt, W. G.; Tracy, C. M.; Barrow, J. R.; Willardson, B. M.; Hartley, J. M.; Tsosie, N. H. Ultrasonic gene and drug delivery using eLiposomes. J. Control. Release, 2013, 167(1), 92-100.

[37] Lattin, J. R.; Belnap, D. M.; Pitt, W. G. Formation of eLiposomes as a drug delivery vehicle. Colloids Surf., B, 2012, 89, 93-100.

[38] Lattin, J. R.; Pitt, W. G.; Belnap, D. M.; Husseini, G. A. Ultrasoundinduced calcein release from eLiposomes. Ultrasound Med. Biol., 2012, 38(12), 2163-73.

[39] Lin, C. Y.; Javadi, M.; Belnap, D. M.; Barrow, J. R.; Pitt, W. G. Ultrasound sensitive eLiposomes containing doxorubicin for drug targeting therapy. Nanomed., 2014, 10, 67-76.

[40] Huang, S. L.; MacDonald, R. C. Acoustically active liposomes for drug encapsulation and ultrasound-triggered release. Biochim. Biophys. Acta, 2004, 1665(1-2), 134-41.

[41] Zhao, Y. Z.; Du, L. N.; Lu, C. T.; Jin, Y. G.; Ge, S. P. Potential and problems in ultrasound-responsive drug delivery systems. Int. J. Nanomedicine, 2013, 8, 1621-33.

[42] Deshpande, P. P.; Biswas, S.; Torchilin, V. P. Current trends in the use of liposomes for tumor targeting. Nanomedicine (Lond), 2013, 8(9), 1509-28.

[43] Schwan, H. P. Electromagnetic and ultrasonic induction of hyperthermia in tissue-like substances. Radiat. Environ. Biophys., 1980, 17(3), 189-203.

[44] Amstad, E.; Kohlbrecher, J.; Muller, E.; Schweizer, T.; Textor, M.; Reimhult, E. Triggered release from liposomes through magnetic 
actuation of iron oxide nanoparticle containing membranes. Nano Lett., 2011, 11(4), 1664-70.

[45] Dicheva, B. M.; Koning, G. A. Targeted thermosensitive liposomes: an attractive novel approach for increased drug delivery to solid tumors. Expert Opin. Drug Deliv., 2014, 11(1), 83-100.

[46] Grull, H.; Langereis, S. Hyperthermia-triggered drug delivery from temperature-sensitive liposomes using MRI-guided high intensity focused ultrasound. J. Control. Release, 2012, 161(2), 317-27.

[47] Kneidl, B.; Peller, M.; Winter, G.; Lindner, L. H.; Hossann, M. Thermosensitive liposomal drug delivery systems: state of the art review. Int. J. Nanomedicine, 2014, 9, 4387-4398.

[48] Kumar, C. S.; Mohammad, F. Magnetic nanomaterials for hyperthermia-based therapy and controlled drug delivery. Adv Drug Deliv Rev, 2011, 63(9), 789-808.

[49] Ta, T.; Porter, T. M. Thermosensitive liposomes for localized delivery and triggered release of chemotherapy. J. Control. Release, 2013, 169(1â€"2), 112-125.

[50] de Smet, M.; Langereis, S.; van den Bosch, S.; Grull, H. Temperature-sensitive liposomes for doxorubicin delivery under MRI guidance. J. Control. Release, 2010, 143(1), 120-7.

[51] Gasselhuber, A.; Dreher, M. R.; Partanen, A.; Yarmolenko, P. S.; Woods, D.; Wood, B. J.; Haemmerich, D. Targeted drug delivery by high intensity focused ultrasound mediated hyperthermia combined with temperature-sensitive liposomes: computational modelling and preliminary in vivo validation. Int. J. Hyperthermia, 2012, 28(4), $337-48$.

[52] Gasselhuber, A.; Dreher, M. R.; Rattay, F.; Wood, B. J.; Haemmerich, D. Comparison of Conventional Chemotherapy, Stealth Liposomes and Temperature-Sensitive Liposomes in a Mathematical Model. PLoS ONE, 2012, 7(10), e47453.

[53] Lim, S.-J.; Carling, C.-J.; Warford, C. C.; Hsiao, D.; Gates, B. D.; Branda, N. R. Multifunctional photo- and thermo-responsive copolymer nanoparticles. Dyes Pigments, 2011, 89(3), 230-235.

[54] Park, S. M.; Kim, M. S.; Park, S.-J.; Park, E. S.; Choi, K.-S.; Kim, Y.-s.; Kim, H. R. Novel temperature-triggered liposome with high stability: Formulation, in vitro evaluation, and in vivo study combined with high-intensity focused ultrasound (HIFU). J. Control. Release, 2013, 170(3), 373-379.

[55] Deatsch, A. E.; Evans, B. A. Heating efficiency in magnetic nanoparticle hyperthermia. J. Magn. Magn. Mater., 2014, 354(0), 163-172.

[56] Demura, K.; Morikawa, S.; Murakami, K.; Sato, K.; Shiomi, H.; Naka, S.; Kurumi, Y.; Inubushi, T.; Tani, T. An easy-to-use microwave hyperthermia system combined with spatially resolved MR temperature maps: phantom and animal studies. J. Surg. Res., 2006, 135(1), 179-86.

[57] Forbes, N. A.; Zasadzinski, J. A. Localized Photothermal Heating of Temperature Sensitive Liposomes. Biophys. J., 2010, 98(3), 274a.

[58] Ernsting, M. J.; Worthington, A.; May, J. P.; Tagami, T.; Kolios, M. C.; Li, S.-D. In Ultrasound drug targeting to tumors with thermosensitive liposomes, 2011 IEEE Int Ultrasonics Symp., 2011; 2011; pp 1-4.

[59] Yatvin, M. B.; Weinstein, J. N.; Dennis, W. H.; Blumenthal, R. Design of liposomes for enhanced local release of drugs by hyperthermia. Science, 1978, 202(4374), 1290-3.

[60] Gaber, M. H.; Hong, K.; Huang, S. K.; Papahadjopoulos, D. Thermosensitive sterically stabilized liposomes: formulation and in vitro studies on mechanism of doxorubicin release by bovine serum and human plasma. Pharm. Res., 1995, 12(10), 1407-16.

[61] Unezaki, S.; Maruyama, K.; Takahashi, N.; Koyama, M.; Yuda, T.; Suginaka, A.; Iwatsuru, M. Enhanced delivery and antitumor activity of doxorubicin using long-circulating thermosensitive liposomes containing amphipathic polyethylene glycol in combination with local hyperthermia. Pharm. Res., 1994, 11(8), 1180-5.

[62] Needham, D.; Anyarambhatla, G.; Kong, G.; Dewhirst, M. W. A new temperature-sensitive liposome for use with mild hyperthermia: characterization and testing in a human tumor xenograft model. Cancer Res., 2000, 60(5), 1197-201.

[63] Kono, K. Thermosensitive polymer-modified liposomes. Adv Drug Deliv Rev, 2001, 53(3), 307-19.

[64] Kono, K.; Nakai, R.; Morimoto, K.; Takagishi, T. Thermosensitive polymer-modified liposomes that release contents around physiological temperature. Biochim. Biophys. Acta, 1999, 1416(1â€“"2), 239-250.
[65] Schild, H. G. Poly(N-isopropylacrylamide): experiment, theory and application. Prog. Polym. Sci., 1992, 17(2), 163-249.

[66] Kono, K.; Hayashi, H.; Takagishi, T. Temperature-sensitive liposomes: liposomes bearing poly (N-isopropylacrylamide). $J$. Control. Release, 1994, 30(1), 69-75.

[67] Kono, K.; Henmi, A.; Takagishi, T. Temperature-controlled interaction of thermosensitive polymer-modified cationic liposomes with negatively charged phospholipid membranes.

Biochim. Biophys. Acta, 1999, 1421(1), 183-197.

[68] Kono, K.; Henmi, A.; Yamashita, H.; Hayashi, H.; Takagishi, T. Improvement of temperature-sensitivity of poly(Nisopropylacrylamide)-modified liposomes. J. Control. Release, 1999, 59(1), 63-75.

[69] Kim, J.-C.; Kim, J.-D. Release property of temperature-sensitive liposome containing poly(N-isopropylacrylamide). Colloids Surf., $B$, 2002, 24(1), 45-52.

[70] Nishita, T. Heat-sensitive liposomes containing cisplatin and localized hyperthermia in treatment of murine tumor. Osaka City Med. J., 1998, 44(1), 73-83.

[71] Paasonen, L.; Romberg, B.; Storm, G.; Yliperttula, M.; Urtti, A.; Hennink, W. E. Temperature-sensitive poly(N-(2-hydroxypropyl) methacrylamide mono/dilactate)-coated liposomes for triggered contents release. Bioconjug. Chem., 2007, 18(6), 2131-6.

[72] Vollmer, M. Physics of the microwave oven. Physics Education, 2003, 39, 74-81.

[73] Gabriel, C.; Gabriel, S.; Grant, E.; Halstead, B.; Michael, D.; Mingos, P. Dielectric parameters relevant to microwave dielectric heating. Chem. Soc. Rev., 1998, 27, 213-224.

[74] Bradshaw, S. M.; van Wyk, E. J.; de Swardt, J. B. Microwave heating principles and the application to the regeneration of granular activated carbon. The Journal of The South African Institute of Mining and Metallurgy, 1998, July/August 1998, 201212.

[75] van der Heijden, A. G.; Kiemeney, L. A.; Gofrit, O. N.; Nativ, O.; Sidi, A.; Leib, Z.; Colombo, R.; Naspro, R.; Pavone, M.; Baniel, J.; Hasner, F.; Witjes, J. A. Preliminary European results of local microwave hyperthermia and chemotherapy treatment in intermediate or high risk superficial transitional cell carcinoma of the bladder. Eur. Urol., 2004, 46(1), 65-71; discussion 71-2.

[76] Popovic, Z.; Popovic, B. D. In: Introductory Electromagnetics; Horton, M., Ed.; Prentice Hall: New Jersey, 2010; pp 382-392.

[77] Staelin, D. H. In: Electromagnetics and Applications; Staelin, D. H., Ed.; Massachusetts Institute of Technology Cambridge, MA, 2011; pp 255-298.

[78] Vatansever, F.; Hamblin, M. R. Far infrared radiation (FIR): its biological effects and medical applications. Photonics Lasers Med, 2012, 4, 255-266.

[79] Gilchrist, R. K.; Medal, R.; Shorey, W. D.; Hanselman, R. C.; Parrott, J. C.; Taylor, C. B. Selective inductive heating of lymph nodes. Ann. Surg., 1957, 146(4), 596-606.

[80] Babincova, M.; Cicmanec, P.; Altanerova, V.; Altaner, C.; Babinec, P. AC-magnetic field controlled drug release from magnetoliposomes: design of a method for site-specific chemotherapy. Bioelectrochemistry, 2002, 55(1-2), 17-9.

[81] Pradhan, P.; Giri, J.; Rieken, F.; Koch, C.; Mykhaylyk, O.; Doblinger, M.; Banerjee, R.; Bahadur, D.; Plank, C. Targeted temperature sensitive magnetic liposomes for thermochemotherapy. J. Control. Release, 2010, 142(1), 108-21.

[82] Tai, L. A.; Tsai, P. J.; Wang, Y. C.; Wang, Y. J.; Lo, L. W.; Yang, C. $\mathrm{S}$. Thermosensitive liposomes entrapping iron oxide nanoparticles for controllable drug release. Nanotechnology, 2009, 20(13), 135101.

[83] Danhier, F.; Feron, O.; Preat, V. To exploit the tumor microenvironment: Passive and active tumor targeting of nanocarriers for anti-cancer drug delivery. J. Control. Release, 2010, 148(2), 135-146.

[84] de la Rica, R.; Aili, D.; Stevens, M. M. Enzyme-responsive nanoparticles for drug release and diagnostics. Adv Drug Deliv Rev, 2012, 64(11), 967-978.

[85] Arouri, A.; Hansen, A. H.; Rasmussen, T. E.; Mouritsen, O. G. Lipases, liposomes and lipid-prodrugs. Curr. Opin. Colloid Interface Sci., 2013, 18(5), 419-431.

[86] Luk, A. S.; Kaler, E. W.; Lee, S. P. Phospholipase C-induced aggregation and fusion of cholesterol-lecithin small unilamellar vesicles. Biochemistry (Mosc.), 1993, 32(27), 6965-73. 
[87] Nieva, J. L.; Goni, F. M.; Alonso, A. Liposome fusion catalytically induced by phospholipase C. Biochemistry (Mosc.), 1989, 28(18), 7364-7367.

[88] Abe, T.; Sakamoto, K.; Kamohara, H.; Hirano, Y.; Kuwahara, N.; Ogawa, M. Group II phospholipase A2 is increased in peritoneal and pleural effusions in patients with various types of cancer. Int. J. Cancer, 1997, 74(3), 245-50.

[89] Yamashita, S.; Yamashita, J.; Sakamoto, K.; Inada, K.; Nakashima, Y.; Murata, K.; Saishoji, T.; Nomura, K.; Ogawa, M. Increased expression of membrane-associated phospholipase A2 shows malignant potential of human breast cancer cells. Cancer, 1993, 71(10), 3058-64.

[90] Davidsen, J.; Jorgensen, K.; Andresen, T. L.; Mouritsen, O. G. Secreted phospholipase A2 as a new enzymatic trigger mechanism for localised liposomal drug release and absorption in diseased tissue. Biochim. Biophys. Acta, 2003, 1609(1), 95-101.

[91] Davidsen, J.; Vermehren, C.; Frokjaer, S.; Mouritsen, O. G.; Jorgensen, K. Drug delivery by phospholipase A2 degradable liposomes. Int. J. Pharm., 2001, 214(1â€“"2), 67-69.

[92] Kaasgaard, T.; Andresen, T. L.; Jensen, S. S.; Holte, R. O.; Jensen, L. T.; Jorgensen, K. Liposomes containing alkylated methotrexate analogues for phospholipase A2 mediated tumor targeted drug delivery. Chem. Phys. Lipids, 2009, 157(2), 94-103.

[93] Andresen, T. L.; Jensen, S. S.; Jorgensen, K. Advanced strategies in liposomal cancer therapy: problems and prospects of active and tumor specific drug release. Prog. Lipid Res., 2005, 44(1), 68-97.

[94] Chau, Y.; Zhong, J.; Moo-Young, M. In: Comprehensive Biotechnology; 2nd ed.; Moo-Young, M., Ed.; Academic Press: Burlington, 2011; pp 605-624.

[95] de Jonge, M. J. A.; Slingerland, M.; Loos, W. J.; Wiemer, E. A. C.; Burger, H.; Mathijssen, R. H. J.; Kroep, J. R.; den Hollander, M. A. G.; van der Biessen, D.; Lam, M.-H.; Verweij, J.; Gelderblom, H. Early cessation of the clinical development of LiPlaCis, a liposomal cisplatin formulation. Eur. J. Cancer, 2010, 46(16), 3016-3021.

[96] Meers, P. Enzyme-activated targeting of liposomes. Adv Drug Deliv Rev, 2001, 53(3), 265-72.

[97] Powers, J. C.; Gupton, B. F.; Harley, A. D.; Nishino, N.; Whitley, R. J. Specificity of porcine pancreatic elastase, human leukocyte elastase and cathepsin G Inhibition with peptide chloromethyl ketones. Biochim. Biophys. Acta, 1977, 485(1), 156-166.

[98] Sato, T.; Takahashi, S.; Mizumoto, T.; Harao, M.; Akizuki, M.; Takasugi, M.; Fukutomi, T.; Yamashita, J. Neutrophil elastase and cancer. Surg. Oncol., 2006, 15(4), 217-22.

[99] Davis, S. C.; Szoka, F. C., Jr. Cholesterol phosphate derivatives: synthesis and incorporation into a phosphatase and calciumsensitive triggered release liposome. Bioconjug. Chem., 1998, 9(6), 783-92.

[100] Drummond, D. C.; Zignani, M.; Leroux, J. Current status of pHsensitive liposomes in drug delivery. Prog. Lipid Res., 2000, 39(5), 409-60.

[101] Ferreira, D. D. S.; Lopes, S. C.; Franco, M. S.; Oliveira, M. C. $\mathrm{pHsensitive} \mathrm{liposomes} \mathrm{for} \mathrm{drug} \mathrm{delivery} \mathrm{in} \mathrm{cancer} \mathrm{treatment.} \mathrm{Ther}$ Deliv, 2013, 4(9), 1099-123.

[102] Karanth, H.; Murthy, R. S. pH-sensitive liposomes--principle and application in cancer therapy. J. Pharm. Pharmacol., 2007, 59(4), 469-83.

[103] Simoes, S.; Moreira, J.; Fonseca, C.; Duzqunes, N.; Pedroso de Lima, M. On the formulation of $\mathrm{pH}$-sensitive liposomes with long circulation times. Adv Drug Deliv Rev, 2004, 56(7), 947-965.

[104] Yatvin, M. B.; Kreutz, W.; Horwitz, B. A.; Shinitzky, M. pHsensitive liposomes: possible clinical implications. Science, 1980, 210(4475), 1253-5.

[105] Straubinger, R. M.; Duzqunes, N.; Papahadjopoulos, D. pHsensitive liposomes mediate cytoplasmic delivery of encapsulated macromolecules. FEBS Lett., 1985, 179(1), 148-154.

[106] Torchilin, V. Multifunctional and stimuli-sensitive pharmaceutical nanocarriers. Eur. J. Pharm. Biopharm., 2009, 71(3), 431-44.

[107] Carvalho Junior, A. D.; Vieira, F. P.; Melo, V. J.; Lopes, M. T.; Silveira, J. N.; Ramaldes, G. A.; Garnier-Suillerot, A.; PereiraMaia, E. C.; Oliveira, M. C. Preparation and cytotoxicity of cisplatincontaining liposomes. Braz. J. Med. Biol. Res., 2007, 40(8), 1149-57.

[108] de Oliveira, M. C.; Rosilio, V.; Lesieur, P.; Bourgaux, C.; Couvreur, P.; Ollivon, M.; Dubernet, C. pH-sensitive liposomes as a carrier for oligonucleotides: a physico-chemical study of the interaction between DOPE and a 15-mer oligonucleotide in excess water. Biophys. Chem., 2000, 87(2-3), 127-37.

[109] Cullis, P. R.; de Kruijff, B. Lipid polymorphism and the functional roles of lipids in biological membranes. Biochim. Biophys. Acta, 1979, 559(4), 399-420.

[110] Litzinger, D. C.; Huang, L. Phosphatidylethanolamine liposomes: drug delivery, gene transfer and immunodiagnostic applications. Biochim. Biophys. Acta, 1992, 1113(2), 201-27.

[111] Liu, D.; Huang, L. pH-sensitive, plasma-stable liposomes with relatively prolonged residence in circulation. Biochim. Biophys. Acta, 1990, 1022(3), 348-54.

[112] Slepushkin, V. A.; Simoes, S.; Dazin, P.; Newman, M. S.; Guo, L. S.; Pedroso de Lima, M. C.; Duzgunes, N. Sterically stabilized pHsensitive liposomes. Intracellular delivery of aqueous contents and prolonged circulation in vivo. J. Biol. Chem., 1997, 272(4), 2382-8.

[113] Shi, G.; Guo, W.; Stephenson, S. M.; Lee, R. J. Efficient intracellular drug and gene delivery using folate receptor-targeted $\mathrm{pH}$-sensitive liposomes composed of cationic/anionic lipid combinations. J. Control. Release, 2002, 80(1-3), 309-19.

[114] Parente, R. A.; Nir, S.; Szoka, F. C., Jr. pH-dependent fusion of phosphatidylcholine small vesicles. Induction by a synthetic amphipathic peptide. J. Biol. Chem., 1988, 263(10), 4724-30.

[115] Almeida, J. D.; Edwards, D. C.; Brand, C. M.; Heath, T. D. Formation of virosomes from influenza subunits and liposomes. Lancet, 1975, 2(7941), 899-901.

[116] Felber, A. E.; Dufresne, M.-H.; Leroux, J.-C. pH-sensitive vesicles, polymeric micelles, and nanospheres prepared with polycarboxylates. Adv Drug Deliv Rev, 2012, 64(11), 979992.

[117] Chen, G.; Hoffman, A. S. Graft copolymers that exhibit temperatureinduced phase transitions over a wide range of $\mathrm{pH}$. Nature, 1995, 373(6509), 49-52.

[118] Hirotsu, S.; Hirokawa, Y.; Tanaka, T. Volume-phase transitions of ionized N-isopropylacrylamide gels. J. Chem. Phys., 1987, 87(2), 1392-1395.

[119] Bertrand, N.; Simard, P.; Leroux, J.-C. In: Liposomes - Methods in Molecular Biology; Weissig, V., Ed.; Humana Press: Totowa, 2010; Vol. 605, pp 545-558.

[120] Brazdova, B.; Zhang, N.; Samoshin, V. V.; Guo, X. trans2Aminocyclohexanol as a $\mathrm{pH}$-sensitive conformational switch in lipid amphiphiles. Chem. Commun., 2008, (39), 4774-4776.

[121] Liu, X.; Zheng, Y.; Samoshina, N. M.; Franz, A. H.; Guo, X.; Samoshin, V. V. Fliposomes: pH-triggered conformational flip of new trans-2-aminocyclohexanol-based amphiphiles causes instant cargo release in liposomes. J. Liposome Res., 2012, 22(4), 319-28.

[122] Samoshin, A. V.; Veselov, I. S.; Chertkov, V. A.; Yaroslavov, A. A.; Grishina, G. V.; Samoshina, N. M.; Samoshin, V. V. Fliposomes: new amphiphiles based on trans-3, 4-bis(acyloxy)piperidine able to perform a pH-triggered conformational flip and cause an instant cargo release from liposomes. Tetrahedron Lett., 2013, 54(41), 56005604.

[123] Samoshina, N. M.; Liu, X.; Brazdova, B.; Franz, A. H.; Samoshin, V. V.; Guo, X. Fliposomes: pH-Sensitive Liposomes Containing a trans2-morpholinocyclohexanol-Based Lipid That Performs a Conformational Flip and Triggers an Instant Cargo Release in Acidic Medium. Pharmaceutics, 2011, 3(3), 379-405.

[124] Zheng, Y.; Liu, X.; Samoshina, N. M.; Chertkov, V. A.; Franz, A. H.; Guo, X.; Samoshin, V. V. Fliposomes: pH-controlled release from liposomes containing new trans-2-morpholinocyclohexanolbased amphiphiles that perform a conformational flip and trigger an instant cargo release upon acidification. Nat. Prod. Commun., 2012, 7(3), 353-8.

[125] Mamasheva, E.; O'Donnell, C.; Bandekar, A.; Sofou, S. Heterogeneous liposome membranes with $\mathrm{pH}$-triggered permeability enhance the in vitro antitumor activity of folatereceptor targeted liposomal doxorubicin. Mol. Pharm., 2011, 8(6), 2224-32.

[126] Carvalho-Junior, A. D.; Mota, L. G.; Nunan, E. A.; Wainstein, A. J.; Wainstein, A. P.; Leal, A. S.; Cardoso, V. N.; De Oliveira, M. C. Tissue distribution evaluation of stealth $\mathrm{pH}$-sensitive liposomal cisplatin versus free cisplatin in Ehrlich tumor-bearing mice. Life Sci., 2007, 80(7), 659-64.

[127] Leite, E. A.; Lana, A. M.; Junior, A. D.; Coelho, L. G.; De Oliveira, M. C. Acute toxicity study of cisplatin loaded longcirculating and 
$\mathrm{pH}$-sensitive liposomes administered in mice. J. Biomed. Nanotechnol., 2011, 8(2), 229-39.

[128] Leite, E. A.; Souza, C. M.; Carvalho-Junior, A. D.; Coelho, L. G.; Lana, A. M.; Cassali, G. D.; Oliveira, M. C. Encapsulation of cisplatin in long-circulating and $\mathrm{pH}$-sensitive liposomes improves its antitumor effect and reduces acute toxicity. Int. J. Nanomedicine, 2012, 7, 5259-69.

[129] Paliwal, S. R.; Paliwal, R.; Pal, H. C.; Saxena, A. K.; Sharma, P. R.; Gupta, P. N.; Agrawal, G. P.; Vyas, S. P. Estrogen-anchored pHsensitive liposomes as nanomodule designed for site-specific delivery of doxorubicin in breast cancer therapy. Mol. Pharm., 2012 9(1), 176-86.

[130] Ishida, T.; Kirchmeier, M. J.; Moase, E. H.; Zalipsky, S.; Allen, T. $\mathrm{M}$. Targeted delivery and triggered release of liposomal doxorubicin enhances cytotoxicity against human B lymphoma cells. Biochim. Biophys. Acta, 2001, 1515(2), 144-58.

[131] Ishida, T.; Okada, Y.; Kobayashi, T.; Kiwada, H. Development of $\mathrm{pH}$-sensitive liposomes that efficiently retain encapsulated doxorubicin (DXR) in blood. Int. J. Pharm., 2006, 309(1-2), 94100.

[132] Gerasimov, O. V.; Boomer, J. A.; Qualls, M. M.; Thompson, D. H. Cytosolic drug delivery using $\mathrm{pH}$ - and light-sensitive liposomes. $A d v$ Drug Deliv Rev, 1999, 38(3), 317-338.

[133] Leung, S. J.; Romanowski, M. Light-activated content release from liposomes. Theranostics, 2012, 2(10), 1020-36.

[134] Yavlovich, A.; Singh, A.; Blumenthal, R.; Puri, A. A novel class of photo-triggerable liposomes containing $\mathrm{DPPC}: \mathrm{DC}(8,9) \mathrm{PC}$ as vehicles for delivery of doxorubcin to cells. Biochim. Biophys. Acta, 2011, 1808(1), 117-26.

[135] Anderson, V. C.; Thompson, D. H. Triggered release of hydrophilic agents from plasmalogen liposomes using visible light or acid. Biochim. Biophys. Acta, 1992, 1109(1), 33-42.

[136] Thompson, D. H.; Gerasimov, O. V.; Wheeler, J. J.; Rui, Y.; Anderson, V. C. Triggerable plasmalogen liposomes: improvement of system efficiency. Biochim. Biophys. Acta, 1996, 1279(1), 2534.

[137] Bisby, R. H.; Mead, C.; Mitchell, A. C.; Morgan, C. G. Fast laserinduced solute release from liposomes sensitized with photochromic lipid: effects of temperature, lipid host, and sensitizer concentration. Biochem. Biophys. Res. Commun., 1999, 262(2), 40610.

[138] Bisby, R. H.; Mead, C.; Morgan, C. G. Wavelength-programmed solute release from photosensitive liposomes. Biochem. Biophys. Res. Commun., 2000, 276(1), 169-73.

[139] Yavlovich, A.; Singh, A.; Tarasov, S.; Capala, J.; Blumenthal, R.; Puri, A. Design of liposomes containing photopolymerizable phospholipids for triggered release of contents J. Therm. Anal. Calorim., 2009, 98(1), 97-104.

[140] Aygun, A.; Torrey, K.; Kumar, A.; Stephenson, L. D. Investigation of factors affecting controlled release from photosensitive DMPC and DSPC liposomes. Appl. Biochem. Biotechnol., 2012, 167(4), 743-57.

[141] Paasonen, L.; Laaksonen, T.; Johans, C.; Yliperttula, M.; Kontturi, K.; Urtti, A. Gold nanoparticles enable selective light-induced contents release from liposomes. J. Control. Release, 2007, 122(1), 86-93.

[142] Mady, M. M.; Fathy, M. M.; Youssef, T.; Khalil, W. M. Biophysical characterization of gold nanoparticles-loaded liposomes. Phys. Med. 2012, 28(4), 288-95.

[143] Paasonen, L.; Sipila, T.; Subrizi, A.; Laurinmaki, P.; Butcher, S. J.; Rappolt, M.; Yaghmur, A.; Urtti, A.; Yliperttula, M. Goldembedded photosensitive liposomes for drug delivery: Triggering mechanism and intracellular release. J. Control. Release, 2010, 147(1), 136-143.

[144] Zhang, Z.; Wang, J.; Chen, C. Near-Infrared Light-Mediated Nanoplatforms for Cancer Thermo-Chemotherapy and Optical Imaging. Adv. Mater., 2013, 25(28), 3869-3880.

[145] Carter, K. A.; Shao, S.; Hoopes, M. I.; Luo, D.; Ahsan, B.; Grigoryants, V. M.; Song, W.; Huang, H.; Zhang, G.; Pandey, R. K.; Geng, J.; Pfeifer, B. A.; Scholes, C. P.; Ortega, J.; Karttunen, M.; Lovell, J. F. Porphyrin-phospholipid liposomes permeabilized by near-infrared light. Nat. Commun., 2014, 5 .

[146] Puri, A. Phototriggerable Liposomes: Current Research and Future Perspectives. Pharmaceutics, 2014, 6(1), 1-25.

[147] Shum, P.; Kim, J.-M.; Thompson, D. H. Phototriggering of liposomal drug delivery systems. Adv Drug Deliv Rev, 2001, 53(3), 273-284.

[148] Lavi, A.; Weitman, H.; Holmes, R. T.; Smith, K. M.; Ehrenberg, B. The depth of porphyrin in a membrane and the membrane's physical properties affect the photosensitizing efficiency. Biophys. J., 2002, 82(4), 2101-10.

[149] Xu, Q.; Geng, S.; Dai, Y.; Qin, G.; Wang, J. Y. CDBA-liposome as an effective sunscreen with longer UV protection and longer shelf life. J. Photochem. Photobiol. B, 2013, 129, 78-86.

[150] Subramaniam, R.; Xiao, Y.; Li, Y.; Qian, S. Y.; Sun, W.; Mallik, S. Light-mediated and H-bond facilitated liposomal release: the role of lipid head groups in release efficiency. Tetrahedron Lett., 2010, 51(3), 529-532.

[151] Freeman, M. W.; Arrott, A.; Watson, J. H. L. Magnetism in Medicine. J. Appl. Phys., 1960, 31(5), S404-S405.

[152] Hayashi, K.; Ono, K.; Suzuki, H.; Sawada, M.; Moriya, M.; Sakamoto, W.; Yogo, T. High-Frequency, MagneticFieldResponsive Drug Release from Magnetic Nanoparticle/Organic Hybrid Based on Hyperthermic Effect. ACS Appl. Mater. Interfaces, 2010, 2(7), 1903-1911.

[153] Hergt, R.; Dutz, S. Magnetic particle hyperthermia - biophysical limitations of a visionary tumour therapy. J. Magn. Magn. Mater., 2007, 311(1), 187-192.

[154] Hoare, T.; Timko, B. P.; Santamaria, J.; Goya, G. F.; Irusta, S.; Lau, S.; Stefanescu, C. F.; Lin, D.; Langer, R.; Kohane, D. S. Magnetically Triggered Nanocomposite Membranes: A Versatile Platform for Triggered Drug Release. Nano Lett., 2011, 11(3), 1395-1400.

[155] Liu, T.-Y.; Liu, K.-H.; Liu, D.-M.; Chen, S.-Y.; Chen, I. W. Temperature-Sensitive Nanocapsules for Controlled Drug Release Caused by Magnetically Triggered Structural Disruption. Adv. Funct. Mater., 2009, 19(4), 616-623.

[156] Hayashi, K.; Nakamura, M.; Miki, H.; Ozaki, S.; Abe, M.; Matsumoto, T.; Sakamoto, W.; Yogo, T.; Ishimura, K. Magnetically responsive smart nanoparticles for cancer treatment with a combination of magnetic hyperthermia and remote-control drug release. Theranostics, 2014, 4(8), 834-44.

[157] Hayashi, K.; Nakamura, M.; Sakamoto, W.; Yogo, T.; Miki, H.; Ozaki, S.; $\quad$ Abe, $\quad$ M.; $\quad$ Matsumoto, T.; Ishimura, K

Superparamagnetic nanoparticle clusters for cancer theranostics combining magnetic resonance imaging and hyperthermia treatment. Theranostics, 2013, 3(6), 366-76.

[158] Soenen, S. J.; Hodenius, M.; De Cuyper, M. Magnetoliposomes: versatile innovative nanocolloids for use in biotechnology and biomedicine. Nanomedicine (Lond), 2009, 4(2), 177-91.

[159] Behnia, S.; Ghalichi, F.; Bonabi, A.; Jafari, A. Ultrasound Thermotherapy of Breast: Theoretical Design of Transducer and Numerical Simulation of Procedure. Jpn J Appl Phys, 2006, 45(3R), 1856.

[160] Carson, P. L.; Fenster, A. Anniversary paper: evolution of ultrasound physics and the role of medical physicists and the AAPM and its journal in that evolution. Med. Phys., 2009, 36(2), 411-28.

[161] Cui, Y.; Peng, J. U. E.; Tang, H. U.; Wang, T.; Chen, S. New focused ultrasound transducer for fat cell disruption J Innov Opt Health Sci, 2012, 05(01), 1250001

[162] de Lucena, C. E.; Dos Santos Junior, J. L.; de Lima Resende, C. A.; do Amaral, V. F.; de Almeida Barra, A.; Reis, J. H. Ultrasoundguided core needle biopsy of breast masses: How many cores are necessary to diagnose cancer? J. Clin. Ultrasound, 2007, 35(7), 363-6.

[163] Kang, T.; Horton, L.; Emery, P.; Wakefield, R. J. Value of ultrasound in rheumatologic diseases. J. Korean Med. Sci., 2013, 28(4), 497507.

[164] Rippey, J. C. R.; Royse, A. G. Ultrasound in trauma. Best Pract Res Cl Anaesthesiol, 2009, 23(3), 343-362.

[165] Abu-Zidan, F. M.; Hefny, A. F.; Corr, P. Clinical ultrasound physics. J. Emerg. Trauma Shock, 2011, 4(4), 501-3.

[166] Arthurs, G. In: AAGBI Core Topics in Anaesthesia; Johnston, I.; Harrop-Griffiths, W.; Gemmell, L., Ed.; Wiley-Blackwell: Oxford, 2011; pp 1-16.

[167] Buddemeyer, E. U. The physics of diagnostic ultrasound. Radiol. Clin. North Am., 1975, 13(3), 391-402.

[168] Frankel, H. L.; deBoisblanc, B. P.; Murthi, S.; Ferguson, M.; Sisley, A. In: Bedside Procedures for the Intensivist; Frankel, H., Ed.; Springer New York: 2010; pp 57-80.

[169] Shriki, J. Ultrasound Physics. Crit. Care Clin., 2014, 30(1), 1-24.

[170] Coltrera, M. D. Ultrasound physics in a nutshell. Otolaryngol. Clin. North Am., 2010, 43(6), 1149-59. 
[171] Edwards, H. Ultrasound Physics and Technology: How, Why and When. Ultrasound, 2010, 18(2), 100.

[172] Baird, R. N. Doppler ultrasound - physics, instrumentation and clinical applications. Br. J. Surg., 1990, 77(3), 355-355.

[173] Hendee, W. R.; Ritenour, E. R. Medical Imaging Physics. 4th Edition ed.; John Wiley \& Sons, Inc.: 2003.

[174] Azhari, H. Basics of Biomedical Ultrasound for Engineers. John Wiley and Sons, Inc.: Hoboken, New Jersey, 2010.

[175] Dalecki, D. Mechanical bioeffects of ultrasound. Annu. Rev. Biomed. Eng., 2004, 6, 229-48.

[176] Liu, H.-L.; Hsieh, C.-M. Single-transducer dual-frequency ultrasound generation to enhance acoustic cavitation. Ultrason. Sonochem., 2009, 16(3), 431-438.

[177] Vadivelu, N.; Urman, R. D.; Hines, R. L.; Halaszynski, T. In: Essentials of Pain Management; Ed.; Springer New York: 2011; pp 469-500.

[178] Ziegler, L.; O'Brien, R. T. Harmonic ultrasound: a review. Vet. Radiol. Ultrasound, 2002, 43(6), 501-9.

[179] Aldrich, J. E. Basic physics of ultrasound imaging. Crit. Care Med., 2007, 35(5 Suppl), S131-7.

[180] Carlsen, E. N. Ultrasound physics for the physician. A brief review. J. Clin. Ultrasound, 1975, 3(1), 69-75.

[181] Hall, D. O.; Selfridge, A. R. Multi-frequency ultrasound therapy systems and methods. 1995.

[182] Ahmadi, F.; McLoughlin, I. V.; Chauhan, S.; ter-Haar, G. Bioeffects and safety of low-intensity, low-frequency ultrasonic exposure. Prog. Biophys. Mol. Biol., 2012, 108(3), 119-38.

[183] Kossoff, G. Basic Physics and Imaging Characteristics of Ultrasound. World J. Surg., 2000, 24(2), 134-142.

[184] Belmonte, R.; Tejero, M.; Ferrer, M.; Muniesa, J. M.; Duarte, E.; Cunillera, O.; Escalada, F. Efficacy of low-frequency low-intensity electrotherapy in the treatment of breast cancer-related lymphoedema: a cross-over randomized trial. Clin. Rehabil., 2012, 26(7), 607-18.

[185] Chen, G. S.; Liu, H. C.; Lin, Y. C.; Lin, Y. L. Experimental analysis of 1-3 piezocomposites for high-intensity focused ultrasound transducer applications. IEEE Trans. Biomed. Eng., 2013, 60(1), 12834.

[186] Horvath, D.; Lesica, N. A. The effects of interaural time difference and intensity on the coding of low-frequency sounds in the mammalian midbrain. J. Neurosci., 2011, 31(10), 3821-7.

[187] Jenne, J. W.; Preusser, T.; Gunther, M. High-intensity focused ultrasound: principles, therapy guidance, simulations and applications. Z Med Phys, 2012, 22(4), 311-22.

[188] Khedr, E. M.; Gilio, F.; Rothwell, J. Effects of low frequency and low intensity repetitive paired pulse stimulation of the primary motor cortex. Clin. Neurophysiol., 2004, 115(6), 1259-63.

[189] Man, J.; Shelton, R. M.; Cooper, P. R.; Scheven, B. A. Lowintensity low-frequency ultrasound promotes proliferation and differentiation of odontoblast-like cells. J. Endod., 2012, 38(5), 608-13.

[190] Buldakov, M. A.; Hassan, M. A.; Zhao, Q. L.; Feril, L. B., Jr.; Kudo, N.; Kondo, T.; Litvyakov, N. V.; Bolshakov, M. A.; Rostov, V. V.; Cherdyntseva, N. V.; Riesz, P. Influence of changing pulse repetition frequency on chemical and biological effects induced by lowintensity ultrasound in vitro. Ultrason. Sonochem., 2009, 16(3), 3927.

[191] Karshafian, R.; Bevan, P. D.; Williams, R.; Samac, S.; Burns, P. N. Sonoporation by ultrasound-activated microbubble contrast agents: effect of acoustic exposure parameters on cell membrane permeability and cell viability. Ultrasound Med. Biol., 2009, 35(5), 847-60.

[192] Choi, J. J.; Selert, K.; Gao, Z.; Samiotaki, G.; Baseri, B.; Konofagou, E. E. Noninvasive and localized blood-brain barrier disruption using focused ultrasound can be achieved at short pulse lengths and low pulse repetition frequencies. J. Cereb. Blood Flow Metab., 2011, 31(2), 725-37.

[193] Lieu, D. Ultrasound physics and instrumentation for pathologists. Arch. Pathol. Lab. Med., 2010, 134(10), 1541-56.

[194] Pua, E. C.; Zhong, P. Ultrasound-mediated drug delivery. IEEE Eng Med Biol 2009, 28(1), 64-75.

[195] Rapoport, N. Ultrasound-mediated micellar drug delivery. Int. J. Hyperthermia, 2012, 28(4), 374-385.

[196] Wagner, D. R. Ultrasound as a Tool to Assess Body Fat. Journal of Obesity, 2013, 2013, 9.
[197] de Smet, M.; Heijman, E.; Langereis, S.; Hijnen, N. M.; Grull, H. Magnetic resonance imaging of high intensity focused ultrasound mediated drug delivery from temperature-sensitive liposomes: an in vivo proof-of-concept study. J. Control. Release, 2011, 150(1), 10210.

[198] Li, L.; ten Hagen, T. L.; Hossann, M.; Suss, R.; van Rhoon, G. C.; Eggermont, A. M.; Haemmerich, D.; Koning, G. A. Mild hyperthermia triggered doxorubicin release from optimized stealth thermosensitive liposomes improves intratumoral drug delivery and efficacy. J. Control. Release, 2013, 168(2), 142-50.

[199] Chen, D.; Wu, J. An in vitro feasibility study of controlled drug release from encapsulated nanometer liposomes using high intensity focused ultrasound. Ultrasonics, 2010, 50(8), 744-9.

[200] Lagendijk, J. J.; Crezee, J.; Hand, J. W. Dose uniformity in scanned focused ultrasound hyperthermia. Int. J. Hyperthermia, 1994, 10(6), $775-84$

[201] Lalonde, R. J.; Hunt, J. W. Optimizing ultrasound focus distributions for hyperthermia. IEEE Trans. Biomed. Eng., 1995, 42(10), 981-990.

[202] Wang, S.; Frenkel, V.; Zderic, V. Optimization of pulsed focused ultrasound exposures for hyperthermia applications. J. Acoust. Soc. Am., 2011, 130(1), 599-609.

[203] Tole, N. M. In: Basic Physics of Ultrasonographic Imaging.; Ostensen, H., Ed.; World Health Organization.: 2005.

[204] Damianou, C. MRI monitoring of the effect of tissue interfaces in the penetration of high intensity focused ultrasound in kidney in vivo. Ultrasound Med. Biol., 2004, 30(9), 1209-15.

[205] Baronzio, G. F.; Hager, E. D. Hyperthermia in Cancer Treatment: A Primer. Landes Bioscience and Springer Science+Business Media, LLC: Boston, MA, 2006.

[206] Saniei, N. Hyperthermia and Cancer Treatment. Heat Transfer Eng., 2009, 30(12), 915-917.

[207] Wust, P.; Hildebrandt, B.; Sreenivasa, G.; Rau, B.; Gellermann, J.; Riess, H.; Felix, R.; Schlag, P. M. Hyperthermia in combined treatment of cancer. Lancet Oncol., 2002, 3(8), 487-97.

[208] Leighton, T. G. What is ultrasound? Prog. Biophys. Mol. Biol., 2007, 93(1-3), 3-83.

[209] Bilah, K. Y.; Scanian, R. H. Resonance, Tacoma Narrows bridge failure and undergraduate physics textbooks. Am. J. Phys., 1991, 59 , 118-124.

[210] Wolfrum, B. Cavitation and Shock Wave Effects on Biological Systems. University of Gottingen Gottingen, 2004.

[211] Afadzi, M.; Strand, S. P.; Nilssen, E. A.; Masoy, S. E.; Johansen, T. F.; Hansen, R.; Angelsen, B. A.; de, L. D. C. Mechanisms of the ultrasound-mediated intracellular delivery of liposomes and dextrans. IEEE Trans Ultrason Ferroelectr Freq Control, 2013, 60(1), 21-33.

[212] Diaz de la Rosa, M. A.; Husseini, G. A.; Pitt, W. G. Mathematical modeling of microbubble cavitation at $70 \mathrm{kHz}$ and the importance of the subharmonic in drug delivery from micelles. Ultrasonics, 2012, 53(1), 97-110.

[213] Hernot, S.; Klibanov, A. L. Microbubbles in ultrasound-triggered drug and gene delivery. Adv Drug Deliv Rev, 2008, 60(10), 115366.

[214] Zhong, P.; Zhou, Y.; Zhu, S. Dynamics of bubble oscillation in constrained media and mechanisms of vessel rupture in SWL. Ultrasound Med. Biol., 2001, 27(1), 119-34.

[215] Pitt, W. G.; Husseini, G. A.; Staples, B. J. Ultrasonic drug delivery-a general review. Expert Opin. Drug Deliv., 2004, 1(1), 37-56.

[216] Wu, J.; Nyborg, W. L. Ultrasound, cavitation bubbles and their interaction with cells. Adv Drug Deliv Rev, 2008, 60(10), 1103-16.

[217] Diaz-De-La-Rosa, M. A. High-frequency ultrasound drug delivery and cavitation. Brigham Young University: Provo, UT, 2007.

[218] Huang, S. L. Liposomes in ultrasonic drug and gene delivery. $A d v$ Drug Deliv Rev, 2008, 60(10), 1167-76.

[219] Church, C. C.; Yang, X. The mechanical index and cavitation in tissue. J. Acoust. Soc. Am., 2005, 117(4), 2530-2530.

[220] Franc, J.-P.; Michel, J.-M. Fundamentals of Cavitation. Springer Science + Business Media, Inc.: Dordrecht, 2005.

[221] Mehier-Humbert, S.; Yan, F.; Frinking, P.; Schneider, M.; Guy, R. H.; Bettinger, T. Ultrasound-mediated gene delivery: influence of contrast agent on transfection. Bioconjug. Chem., 2007, 18(3), 65262.

[222] Oerlemans, C.; Deckers, R.; Storm, G.; Hennink, W. E.; Nijsen, J. F. Evidence for a new mechanism behind HIFU-triggered release from liposomes. J. Control. Release, 2013, 168(3), 327-33.

[223] Boucher, P.-A.; Joos, B.; Zuckermann, M. J.; Fournier, L. Pore Formation in a Lipid Bilayer under a Tension Ramp: Modeling the 
Distribution of Rupture Tensions. Biophys. J., 2007, 92(12), 43444355.

[224] Netz, R. R.; Schick, M. Pore formation and rupture in fluid bilayers. Phys Rev E Stat Phys Plasmas Fluids Relat Interdiscip Topics, 1996, 53(4), 3875-3885

[225] Lin, H. Y.; Thomas, J. L. PEG-Lipids and oligo(ethylene glycol) surfactants enhance the ultrasonic permeabilizability of liposomes. Langmuir, 2003, 19, 1098-1105.

[226] Yuh, E. L.; Shulman, S. G.; Mehta, S. A.; Xie, J.; Chen, L.; Frenkel, V.; Bednarski, M. D.; Li, K. C. Delivery of systemic chemotherapeutic agent to tumors by using focused ultrasound: study in a murine model. Radiology, 2005, 234(2), 431-7.

[227] Dromi, S.; Frenkel, V.; Luk, A.; Traughber, B.; Angstadt, M.; Bur, M.; Poff, J.; Xie, J.; Libutti, S. K.; Li, K. C.; Wood, B. J. Pulsedhigh intensity focused ultrasound and low temperature-sensitive liposomes for enhanced targeted drug delivery and antitumor effect. Clin. Cancer Res., 2007, 13(9), 2722-7.

[228] Schroeder, A.; Avnir, Y.; Weisman, S.; Najajreh, Y.; Gabizon, A.; Talmon, Y.; Kost, J.; Barenholz, Y. Controlling liposomal drug release with low frequency ultrasound: mechanism and feasibility. Langmuir, 2007, 23, 4019-4025.

[229] Schroeder, A.; Honen, R.; Turjeman, K.; Gabizon, A.; Kost, J.; Barenholz, Y. Ultrasound triggered release of cisplatin from liposomes in murine tumors. J. Control. Release, 2009, 137(1), 638.

[230] Pitt, W. G.; Husseini, G. A.; Roeder, B. L.; Dickinson, D. J.; Warden, D. R.; Hartley, J. M.; Jones, P. W. Preliminary results of combining low frequency low intensity ultrasound and liposomal drug delivery to treat tumors in rats. J. Nanosci. Nanotechnol., 2011, 11(3), 186670.

[231] Evjen, T. J.; Hagtvet, E.; Moussatov, A.; Rognvaldsson, S.; Mestas, J. L.; Fowler, R. A.; Lafon, C.; Nilssen, E. A. In vivo monitoring of liposomal release in tumours following ultrasound stimulation. Eur. $J$.

Pharm. Biopharm., 2013, 84(3), 526-31.

[232] Ninomiya, K.; Kawabata, S.; Tashita, H.; Shimizu, N. Ultrasoundmediated drug delivery using liposomes modified with a thermosensitive polymer. Ultrason. Sonochem., 2014, 2l(1), 310316.

[233] Rizzitelli, S.; Giustetto, P.; Boffa, C.; Delli Castelli, D.; Cutrin, J. C.; Aime, S.; Terreno, E. In vivo MRI visualization of release from liposomes triggered by local application of pulsed low-intensity nonfocused ultrasound. Nanomed., 2014.
[234] Wang, P.; Li, Y.; Wang, X.; Guo, L.; Su, X.; Liu, Q. Membrane damage effect of continuous wave ultrasound on K562 human leukemia cells. J. Ultrasound Med., 2012, 31(12), 1977-86.

[235] Cancer Research UK. High intensity focused ultrasound (HIFU) for prostate cancer. http://www.cancerresearchuk.org/about-cancer/ type/prostate-cancer/treatment/high-intensity-focusedultrasoundfor-prostate-cancer (Accessed February 15, 2015).

[236] Hayes, B. T.; Merrick, M. A.; Sandrey, M. A.; Cordova, M. L. Three$\mathrm{MHz}$ Ultrasound Heats Deeper Into the Tissues Than Originally Theorized. J. Athl. Train., 2004, 39(3), 230-234.

[237] Rodgers, C. Questions about Prenatal Ultrasound and the Alarming Increase in Autism. Midwifery Today, 2006, 80.

[238] Ang, E. S. B. C.; Gluncic, V.; Duque, A.; Schafer, M. E.; Rakic, P. Prenatal exposure to ultrasound waves impacts neuronal migration

in mice. Proceedings of the National Academy of Sciences, 2006, 103(34), 12903-12910.

[239] Allen, T. M.; Cullis, P. R. Liposomal drug delivery systems: from concept to clinical applications. Adv Drug Deliv Rev, 2013, 65(1), 3648.

[240] Zhang, L.; Gu, F. X.; Chan, J. M.; Wang, A. Z.; Langer, R. S.; Farokhzad, O. C. Nanoparticles in medicine: therapeutic applications and developments. Clin. Pharmacol. Ther., 2008, 83(5), 761-9.

[241] Babu, A.; Templeton, A. K.; Munshi, A.; Ramesh, R. NanoparticleBased Drug Delivery for Therapy of Lung Cancer: Progress and Challenges. Journal of Nanomaterials, 2013, 2013, 11.

[242] Thigpen, J. T.; Aghajanian, C. A.; Alberts, D. S.; Campos, S. M.; Gordon, A. N.; Markman, M.; McMeekin, D. S.; Monk, B. J.; Rose, P. G. Role of pegylated liposomal doxorubicin in ovarian cancer. Gynecol. Oncol., 2005, 96(1), 10-8.

[243] Pillai, G. Nanomedicines for Cancer Therapy: An Update of FDA Approved and Those under Various Stages Development. SOJ Pharm Pharm Sci, 2014, 1(2), 1-13.

[244] Koudelka, S.; Turanek, J. Liposomal paclitaxel formulations. $J$. Control. Release, 2012, 163(3), 322-34.

[245] Nehate, C.; Jain, S.; Saneja, A.; Khare, V.; Alam, N.; Dubey, R. D.; Gupta, P. N. Paclitaxel formulations: challenges and novel delivery options. Curr. Drug Deliv., 2014, 11(6), 666-86. 